FORWARD SCATTERING OF A PULSED CONTINUOUS WAVE SIGNAL THROUGH LAMINAR AND TURBULENT THERMAL PLUMES

by

\author{
Stephen Gerard Bowen \\ BSEE, United States Naval Academy (1986) \\ Submitted in partial fulfillment of the \\ requirements for the degree of \\ OCEAN ENGINEER \\ at the \\ MASSACHUSETTS INSTITUTE OF TECHNOLOGY \\ and the \\ WOODS HOLE OCEANOGRAPHIC INSTITUTION
}

September 1993

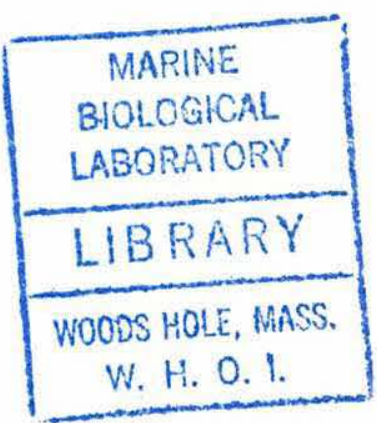

CStephen Gerard Bowen, 1993

The author hereby grants M.I.T. and WHOI permission to reproduce and to distribute copies of this thesis degament in whole or in part.

Signature of Author

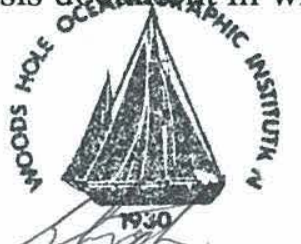

Joint Program in Applied Ocean Science and Engineering

Massachusetts Institute of Technology

Woods Hole Oceanographic Institution

Certified by

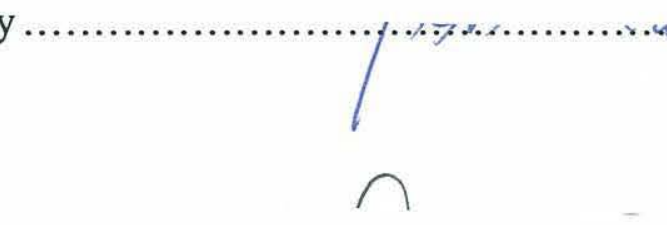

Dr. Josko Catipovic Associate Scientist Woods Hole Oceanographic Institution

Accepted

by

Dr. Arthur B. Baggeroer

Chairman, Joint Committee for Applied Ocean Science and Engineering Massachusetts Institute of Technology / Woods Hole Oceanographic Institution 


\title{
FORWARD SCATTERING OF A PULSED CONTINUOUS WAVE SIGNAL THROUGH LAMINAR AND TURBULENT THERMAL PLUMES
}

\author{
by \\ Stephen Gerard Bowen \\ Submitted to the Massachusetts Institute of Technology/ \\ Woods Hole Oceanographic Institution \\ Joint Program in Applied Ocean Science and Engineering \\ in partial fulfillment of the \\ requirements for the degree of \\ Ocean Engineer
}

\section{Abstract}

The results of an experiment examining the forward propagation of an acoustic signal through a buoyant plume are discussed. Two distinct testing sights were used. One made use of a small fresh water tank in NUWC to provide a controlled plume. The other used a larger salt water tank at WHOI to create a more realistic oceanic model. Using the Born and Rytov approximations, an estimation of the effects of the laminar plume on the propagated signal are shown. As the plume moves from laminar to turbulent, the scintillation index and the Fourier transform of the magnitude square response provide insight into the nature of the transition. Finally, from the turbulent response a model for the scattering function is developed.

Thesis Supervisor: Dr. Josko Catipovic Associate Scientist Woods Hole Oceanographic Institution 


\section{Acknowledgments}

I would like to thank a great number of people who enabled me to enjoy my participation in the M.I.T./WHOI Joint Program. I would also like to thank the U. S. Navy for providing me with this opportunity to study at M.I.T and WHOI.

I owe great thanks to Josko Catipovic and Art Baggeroer who provided me with the guidance necessary to complete this thesis. More importantly, they allowed me to gain a great deal of insight into the nature of their profession.

In order to complete the experiments for this thesis I had to rely a great deal on the generosity of people at WHOI and NUWC. At WHOI: Tim Stanton allowed me the use of his tank; Bob Eastwood eagerly answered all of my silly questions; and Karlen Wannop provided tools and expertise. At NUWC: Lou Goodman allowed me the use of his tank; John Oeschger provided a great deal insight into the expectations of the experiment; and Pete Hebda and Diane Medeiros ensured me the use of the tank and patiently assisted in obtaining and processing the data.

I would also like to thank Matthew Johnson for the ants and Jon Durant for the printer.

Finally, I would like to thank Debbie, Nicholas, and Gillian without whom this would not have been nearly as much fun. 


\section{Contents}

1. Introduction 9

1.1 Statement of Problem......................................... 9

1.2 Overview of Acoustic Turbulent Studies............................. 9

2. Experimental Description 14

$2.1 \quad$ Fresh Water System ...................................... 15

$2.2 \quad$ Salt Water System ........................................ 20

$\begin{array}{ll}\text { 3. Theory } & 23\end{array}$

$3.1 \quad$ Buoyant Plume............................................. 23

3.2 Signal Description ........................................... 27

3.3 Interaction of Signal and Plume................................29

3.3.1 Wave Equation ....................................29

3.3.2 Born Approximation................................. 32

3.3.3 Rytov Approximation ................................ 33

3.4 Cylindrical Approximation....................................... 36

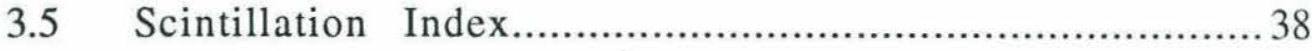

4. Experimental Results 40

4.1 Fresh Water Tank ..................................... 40

4.1.1 Magnitude and Phase Mean Response .................. 41

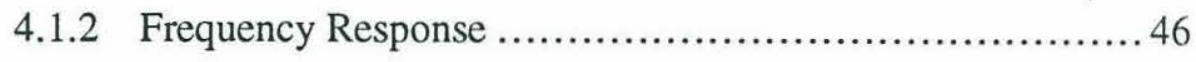

4.1.3 Scintillation Index .................................. 49

4.2 Comparison to Salt Water Tank Data............................ 52

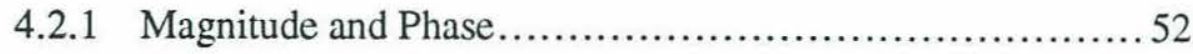

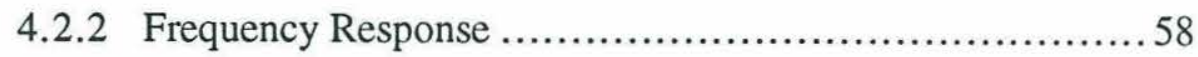

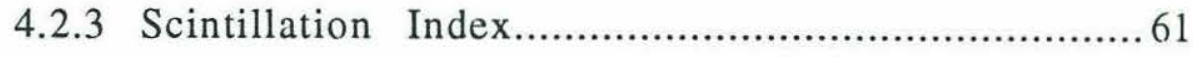


4.3 The Scattering Function............................................ 63

5. Conclusions and Recommendations 68

5.1 Conclusions Concerning Application of Results...................... 68

5.2 Recommendations for Further Research ...........................69 69

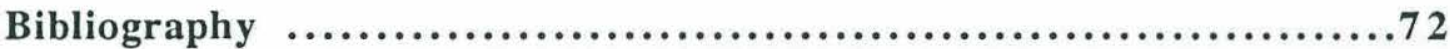




\section{List of Figures}

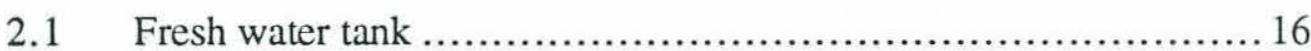

2.2 Plot of heater power vs. variac voltage.............................. 17

2.3 Directionality vs. Perpendicular Range for $1 \mathrm{MHz} 3 / 4$ " source....... 18

2.4 Directionality vs. Perpendicular Range for $1 \mathrm{MHz} 1 / 2$ " source...... 20

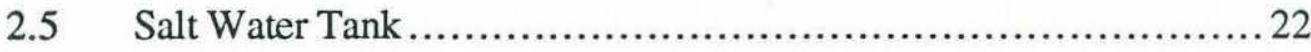

3.1 Isotherm contours of mean turbulent flow......................26

3.2 Sample received signal .......................................... 28

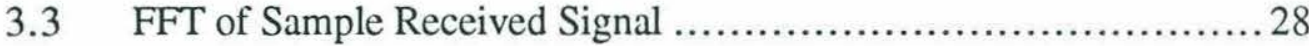

3.4 Rectangular and Cylindrical coordinates ........................ 32

3.5 Magnitude and Phase of Born approximation .................... 33

3.6 Magnitude and Phase of Rytov approximation.................. 36

3.7 Magnitude and Phase of Cylinder approximation.................. 37

$4.1 \quad$ Fresh Water tank Temperature Response ....................... 40

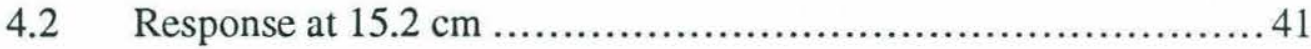

4.3 Response for Born, Rytov, and Experiment ................... 42

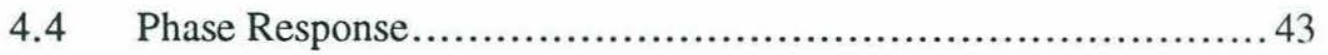

4.5 Magnitude Response for Different Heights........................ 44

4.6 Equivalent Cylinder Temperatures.............................. 44

4.7 Plot of Equivalent Cylinder Temperatures for low powers ......... 45

4.8 Equivalent Cylindrical Temperature vs. Height for maximum power 46

4.9 Propagated Signal in Time.................................. 47

4.10 Propagated Signal in Frequency ............................... 47

4.11 Fourier Transform of Magnitude Response with $\chi 2$ overlay........ 48

4.12 Propagated signal in Frequency for Several Powers ................ 49

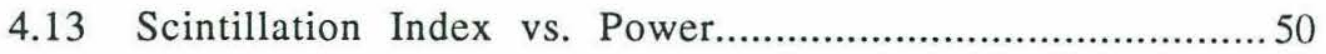


4.14 Received Amplitudes for 43.8 Watt Heater Power Pulse Train ....... 53

4.15 Salt Water Tank Magnitude Plot of $5 \mathrm{~cm}$ data ....................5 53

4.16 Magnitude plot of $5 \mathrm{~cm}$ data ............................... 54

4.17 Phase Plot of $5 \mathrm{~cm}$ data ...................................... 54

4.18 Magnitude Plot of $15 \mathrm{~cm}$ data with Born and Rytov....................55

4.19 Phase plot of $15 \mathrm{~cm}$ data ........................................ 56

4.20 Magnitude plot of $30 \mathrm{~cm}$ data..........................................56

4.21 Phase plot of $30 \mathrm{~cm}$ data ................................... 57

4.22 Resolved Phase plot of $30 \mathrm{~cm}$ data ..............................57

4.23 Fourier Transform of Magnitude Response with $\chi 2$ overlay.........59

4.24 Fourier Transform of Phase Response........................59

4.25 Fourier Transform of Magnitude Response ......................60 60

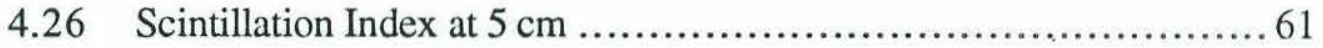

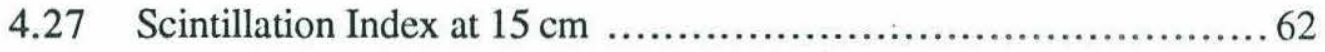

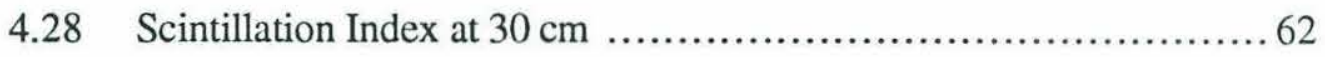

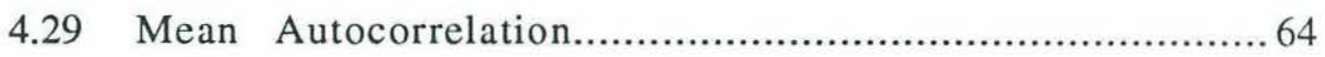

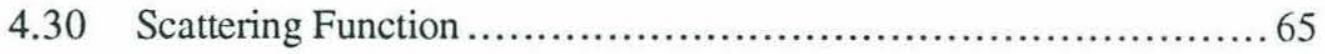

4.31 Scattering Function with Damped Oscillation overlay ..............66

4.32 Log of Scattering Function with Damped Oscillation.............. 66

4.33 Log of Scattering Function with Damped Oscillation (long)........ 67 


\section{Chapter 1}

\section{INTRODUCTION}

\subsection{Statement of Problem}

This thesis examines the effect on a pulsed continuous wave (CW) signal of thermally generated laminar and turbulent plumes. Initially the forward scattering through a laminar plume is modeled using the Born and Rytov approximation. Then a model using scattering from a homogeneous cylinder is used to allow easy comparisons for different thermal profiles as the plume moves from laminar to turbulent. The Fourier transform of the time sequence of the magnitude response squared of the signal train is used to investigate the effective amplitude modulation of the index of refraction. Then, the scintillation index is used to discuss the point at which the plume becomes turbulent. Finally, the scintillation index and Fourier transform of the time sequence magnitude are used to compare data obtained under highly divergent conditions.

\subsection{Overview of Acoustic Turbulent Studies}

The genesis of this research was a discussion concerning a low frequency modulation of a shallow water telemetry signals. The problem was resolved, but the question of the effects of buoyant plumes on line of sight propagation had been raised. Ocean buoyant plumes can arise from a freshwater spring, a heat vent, or even the wake of body moving through a layered medium. These sources vary considerably depending on their extent; the degree to which they alter the effective index of refraction; and the amount of entrained particulate scatterers. In recent years a significant amount of research has concerned observations of hydrothermal vent fields. However, available literature does not indicate as intensive a research into other buoyant plumes. The investigation of these phenomena may be enhanced from the results obtained in this thesis. 
Aside from the observation of the hydrothermal fields, most research on turbulent fields has focused on propagation through turbulent media. Extensive study of electromagnetic wave propagation through turbulent atmosphere exists. ${ }^{1}$ Chernov $^{2}$ and Tatarski ${ }^{3}$ provide the basics of wave interaction with turbulent and random media. Each takes a different approach to similar subjects and if one proves difficult to understand the other will provide the necessary insight. Acoustic propagation has been treated by Wenzel and Keller ${ }^{4}$ and more recently by Yang and McDaniel, ${ }^{5}$ but, in general the electromagnetic literature has been sited and used where appropriate. Flatté discussed the appropriate regions for these applications ${ }^{6}$ to ocean acoustics.

The work of Mintzer in 1953 provides a notable exception to the application of electromagnetics directly to ocean phenomena. Mintzer ${ }^{7}$ attempted to provide a model for the results of experimental work of Sheehy who had used a series of sound pulses to investigate the variation in the propagation of sound in a inhomogeneous media for the Office of Naval Research in 1948. The work is notable not just for the use of pulsed waves but also for the use of "the coefficient of variation;" which has the exact form of the scintillation index except for the use of the absolute value of the pressure field $(|\bar{p}|)$ instead of the intensity (I ).

\footnotetext{
1 Akira Ishimaru, Wave Propagation and Scattering In Random Media, Vol. 2 (New York, San Francisco, London: Academic Press, 1978), Part IV.

${ }^{2}$ Lev A. Chernov, Wave Propagation in A Random Medium, (New York, Toronto, London: McGraw-Hill, 1960). 
$\mathrm{V}^{2}=\frac{\left\langle|\bar{p}|^{2}\right\rangle-|\langle\bar{p}\rangle|^{2}}{|\langle\bar{p}\rangle|^{2}}$

Unfortunately, only propagation through the entire media was considered with no accounting for discrete phenomena. Forward scattering in the ocean through weak fluctuations is also addressed by Duda ${ }^{8}$ with heavy emphasis on the scintillation index. Both Duda and Mintzer look at the increase in the scintillation index (coefficient of variation) with range through the turbulent medium.

There have been several papers that deal directly with turbulence or ocean turbulence as a local phenomena. Rasmussen ${ }^{9}$ discusses the localization of turbulent phenomena based on the reverberation spectrum spreading due to turbulent scatterer motion in contiguous reverberation volumes. The spreading of the spectra discussed involves motion of the scatterers on the order of tens of $\mathrm{cm} / \mathrm{s}$ in order to create a sufficient Doppler frequency. Artel'nyi and Raevskii ${ }^{10}$ look at the effect of multiple scattering of ocean turbulence by looking at the effect of small fluctuations in the sound-velocity-profile. They ignore the temporal fluctuations of the turbulence "since the wave frequency $\omega$ is usually much greater than the characteristic frequencies of the turbulent fluctuations." 11 Instead Artel'nyi and Raevskii concentrate on the effects in the modal spectra.

Papers directly addressing the effect of plumes and turbulent plumes on acoustic propagation have been becoming increasingly frequent in recent years. Among the earliest examples of the work in this field was "The Scattering of Sound in a Turbulent Medium"

\footnotetext{
8 Timothy F. Duda, "Modeling Weak Fluctuations of Undersea Telemetry Signals," IEEE Journal of Oceanic Engineering, 16, No. 1 (January 1991), 3-11.

9 Robert A. Rasmussen, "Remote Detection of Turbulence From Observations of Reverberation Spectra," The Journal of the Acoustical Society of America, 63, No. 1 (January 1978) 101-110.

10 V.V. Artel'nyi and M.A. Raevskii, "Multiple Scattering of Lowfrequency Sound Waves by Ocean Turbulence," Soviet Phys. Acoust., 33, No. 1 (January-February 1987), 4-7.

11 Artel'nyi, p.4.
} 
by Kraichnan ${ }^{12}$ which used turbulent velocity field confined to a finite region and modeled the effect using an isotropic field. The resulting expression for the scattering by isotropic turbulence is:

$$
\langle P(n, \omega)\rangle=\frac{\pi \omega^{4}}{8 c^{8}} \frac{\sin ^{2} 2 \theta}{4 \sin ^{2}\left(\frac{\theta}{2}\right)+\delta^{2}} F\left[\frac{\omega_{\circ}^{2}\left(4 \sin ^{2} \frac{\theta}{2}+\delta^{2}\right)}{c^{2}}, \omega_{\circ} \delta\right]
$$

and, demonstrates that for isotropic turbulence the mean scattered field in the forward direction is zero (0). $\mathrm{F}$ is the ensemble average of the scalar of the frequency wavenumber response of the field: $\mathbf{F}=\left\langle|f(k, \omega)|^{2}\right\rangle$ and $\mathbf{n}$ is the unit vector in a direction from the scatterer. Buoyant plumes may be internally isotropic, but by their very definition as buoyant, imply a variation between the mean internal buoyancy and the surrounding fluid buoyancy. The mean forward scattered signal should contain at least a small variation in the mean field in the forward direction for a buoyant plume.

The off axis scattering of a turbulent plume for forced flow systems has been investigated for flow rates near $650 \mathrm{~cm} / \mathrm{s}$ by Korman ${ }^{13}$ and Beyer. Korman used a modified form of (1) to estimate the received spectrums. The back scattered signal from a particle laden turbulent plume has been investigated by Hay ${ }^{14}$. Hay arrived at a Gaussian particle distribution for the entrained particulates. The Gaussian distribution appears to be a dominating form for many turbulent parameters. Hay's work however concentrated on the backscattered signal from varying particle types and concentration.

12 Robert H. Kraichnan, "The Scattering of Sound in a Turbulent Medium," The Journal of the Acoustical Society of America, 25, No. 6 (November 1953), 1096-1104.

13 M.S. Korman and R. T. Beyer, "The Scattering of Sound by Turbulence in Water, "The Journal of the Acoustical Society of America,67, No. 6 (June 1980), 1980-1987.

14 Alex E. Hay, "Sound Scattering from a Particle Laden, Turbulent Jet," The Journal of the Acoustical Society of America,90, No. 4 (October 1991), 2055-2073. 
Wittek ${ }^{15}$ looked at the amplitude modulation created by turbulent free flow jet at various velocities in the forward scattered direction. By looking at the frequency spectra of the forward scattered field, an estimate of the nature of the turbulence could be obtained. The jet flow velocities used were at a minimum of $2.3 \mathrm{~m} / \mathrm{s}$, almost one hundred times the velocity obtained in this buoyant plume experiment. The frequency spectrum obtained for the forward scattered field intensity closely parallels the results expected in Ishimaru ${ }^{16}$ for a Gaussian velocity distribution with a Doppler shift due to the mean velocity and frequency spreading due to velocity fluctuation.

Goodman and Oeschger ${ }^{17}$ investigated scattering from a buoyant laminar plume in the off axis directions. Further investigation by Oeschger ${ }^{18}$ followed. The temperature profile of a laminar plume was measured and verified to be Gaussian. Then, using the Born approximation, the scattered field was determined in terms of the Bragg wave number and verified by experimental data. The researchers did not investigate forward scattering of the signal through the laminar plume. In addition they did not look at the plume when turbulent or in transition. The laboratory and equipment used in the Goodman and Oeschger experiments were also used in the experiment for this thesis.

An understanding of laminar and turbulent plumes on acoustic signals can be seen, by using the results for the laminar plume obtained by Goodman and Oeschger and extending them to varying power levels. Ultimately, a further understanding of the nature of the transition from laminar to turbulent in terms of their acoustic effects can also be obtained.

\footnotetext{
15 G. Wittek, "Amplitudenmodulation Von Ultraschallwellen durch turbulente Wasserströmungen" Acustica, 27 (1972), 7-14.

16 Ishimaru, p.341.

17 L. Goodman, J. Oeschger, and D. Szargowicz, "Ocean Acoustics Turbulence Study: Acoustic Scattering from a Buoyant Axisymmetric Plume," The Journal of the Acoustical Society of America,91, No. 6 (June 1992), 3212-3227.

18 John W. Oeschger, Acoustic Scattering From a Laminar Plume, University of Rhode Island, 1993.
} 


\section{Chapter 2}

\section{Experimental Description}

The effect of turbulence on the propagation of sound is primarily due to the variation in the effective index of refraction across the plume. For this experiment a heating element is used to create an initially laminar plume which becomes turbulent as power to the plume is increased. An acoustic signal is sent across the plume to study the effects of the plume in the forward scattering direction.

The primary mechanism for the variation in the index of refraction is the variation in the temperature across the plume. From measurements conducted at 60v (11.7 Watts), the laminar plume was found to have the expected Gaussian temperature distribution within a 2 $\mathrm{cm}$ diameter. ${ }^{19}$ Once the plume is turbulent the expected average thermal profile is also Gaussian. ${ }^{20}$ Using these assumptions the variation in the laminar plume with power can be modeled as a cylinder with an effective internal index of refraction.

The turbulent plume creates an effective amplitude modulation of the received signal about a mean value. The extent and form of the turbulence can be determined from the frequency spectrum of the variations in the received signal amplitudes. By using the scintillation index and the frequency spectrum as measures of the variations within the turbulence, a rough estimate of the point at which the plume becomes as turbulent as is possible can be determined.

Two different laboratory set ups were used to investigate the phenomena. One apparatus used the small fresh water tank used by Oeschger and Goodman; this provided a controlled result with a known thermal plume. The other used a large $11.65 \mathrm{~m}^{3}$ salt water

19 Oeschger, p. 36.

20 H. Rouse, C. Yih, and H.W. Humphreys, "Gravitaional Convections from a Boundry Source." Tellus, 4 (1952), 201-210. 
tank which was allowed to stratify and develop internal waves. The internal waves were generated by location in the room and internal and external weather conditions. The latter tank was used to investigate the robustness of the measures of the turbulence.

The two experimental systems completed essentially the same experiment. The face to face distance between the transducers is the only experimental parameter, outside of the water in the tank, that is varied. Both experimental systems allowed the transducers to be placed face to face at specific heights above the heater element. Because the phenomena is discrete and in the far field of the transducers the experiments can be compared directly.

\subsection{Fresh Water System}

A tank made of $2.5 \mathrm{~cm}$ Plexiglas $63.5 \mathrm{~cm}$ wide $118.11 \mathrm{~cm}$ long and $81.28 \mathrm{~cm}$ deep of nominal depth $68 \mathrm{~cm}$ with an open top to allow access to equipment was filled with filtered tap water. Initially filtering used a Barnstead nanopure water filtering system in the gravity feed mode producing a 1.5 liter per minute flow rate while eliminating particles down to 0.2 microns. Eventually, a new filtration system by Pureflow with a 1 micron filtration at 150 liters per minute replaced the old system. The new filtration system was in place for the data set at a height of $15 \mathrm{~cm}$. The systems were turned off during the experiments to prevent distortions in the plume due to filtration flow. The continual flow helped limit stratification of the tank as well as removing particulates.

An anodized aluminum frame with an open internal ring, allowing face to face transducer ranges of $50.96 \mathrm{~cm}$, was placed in the tank. Threaded stainless steel rods attached the ring to the base. The rods allowed variation in the transducer height above the plume. The base held the heating element in the center of the ring. The frame could be removed from the tank to make adjustments. The heating element, $0.95 \mathrm{~cm}$ diameter by $2.5 \mathrm{~cm}$ in height, was rated at 50 watts at 120 volts rms. The analog variable power supply allowed variation from 0 to 140 volts. By varying the power to the heater the plume varies. 


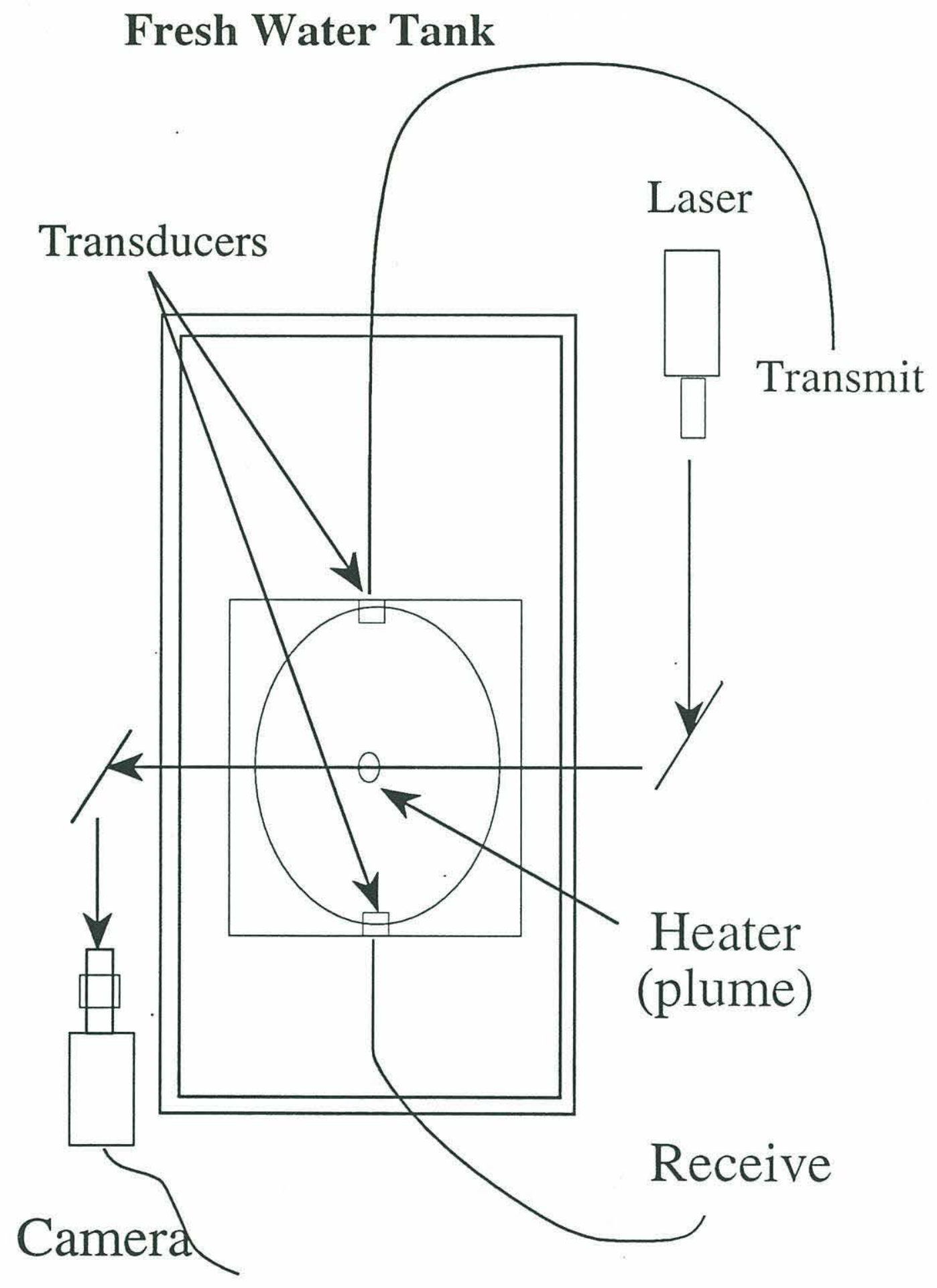

Figure 2.1 


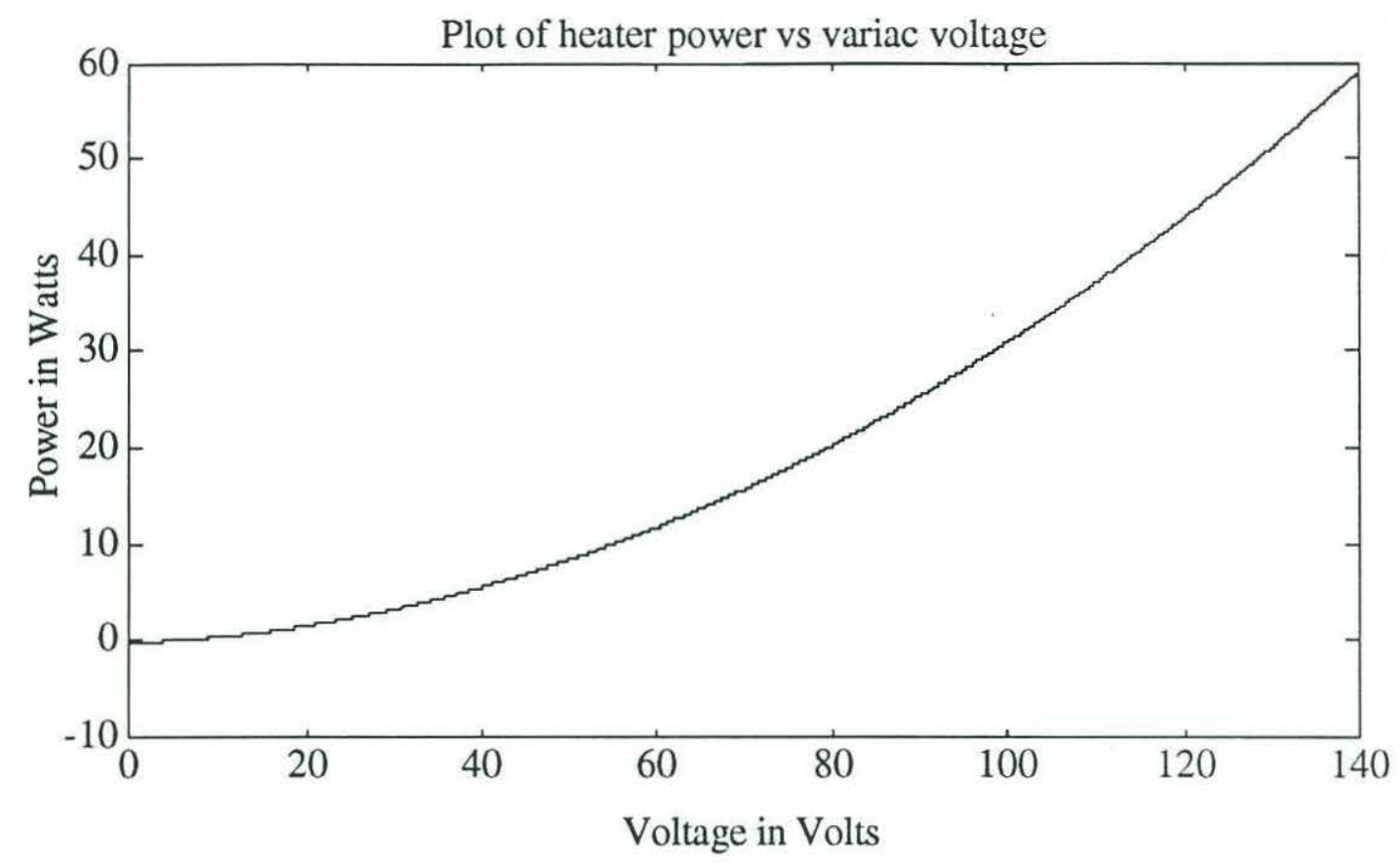

Figure 2.2

The nature of the plume ( laminar, unstable, or turbulent ) needed to be determined easily in order to verify the acoustic results. A laser shadow graph system provided real time qualitative information on the nature of the plume. A 5 milliwatt diode laser having a wavelength of $670 \mathrm{~nm}$ provided the light source. The laser beam was expanded and directed through the plume perpendicular to the acoustic axis and the plume axis. On the opposite side of the tank the beam was directed to a screen from which a video image was recorded, digitized, and displayed using a Raster Ops 364 Color Video board. The digitized signal was saved periodically for latter analysis.

The transducers used in the experiment were 3/4" Panametrics transducers with 1 Mhz center frequencies with a $-10 \mathrm{~dB}$ bandwidth of $400 \mathrm{kHz}$ to $1.4 \mathrm{MHz}$. "Beam pattern data taken on the transducers showed that they could be well modeled by baffled pistons."21 Using a model of the transducer as a plane circular piston the "U.S. Institute (now American National Standards Institute) arbitrarily decided that the criterion for a

21 Oeschger, p.29. 
circular piston will be that the far field begins at the critical range $R_{c}=\mathrm{pa}^{2} / 1$ " 22 for $a=$ transducer radius. This corresponds to a value of $19 \mathrm{~cm}$. Another approach for determining the point at which the far field begins, uses the known expression for the on axis extrema: $r_{m}=\left(a^{2} / m l\right)-(m l / 4)$, and then defines the far-field region as the region beyond the first local maxima. Using this method the distance, $R_{c}$, becomes $6 \mathrm{~cm}$. In both circumstances the distance to the plume is beyond the value of $\mathrm{R}_{\mathrm{c}}$. One final method of evaluating the effectiveness of the far field approximations involves looking at the directionality of the transducer at the range of interest. Using Clay and Medwin 23 the directionality is determined and plotted for the distance perpendicular $\left(\mathrm{r}_{\mathrm{p}}\right)$ to the propagation path:

$\mathrm{D}=\frac{2\left[J_{1}(k \operatorname{asin} \phi)\right]}{k \operatorname{asin} \phi}$

where $\phi=\arctan \left(\mathrm{r}_{\mathrm{p}} / 25 \mathrm{~cm}\right)$.

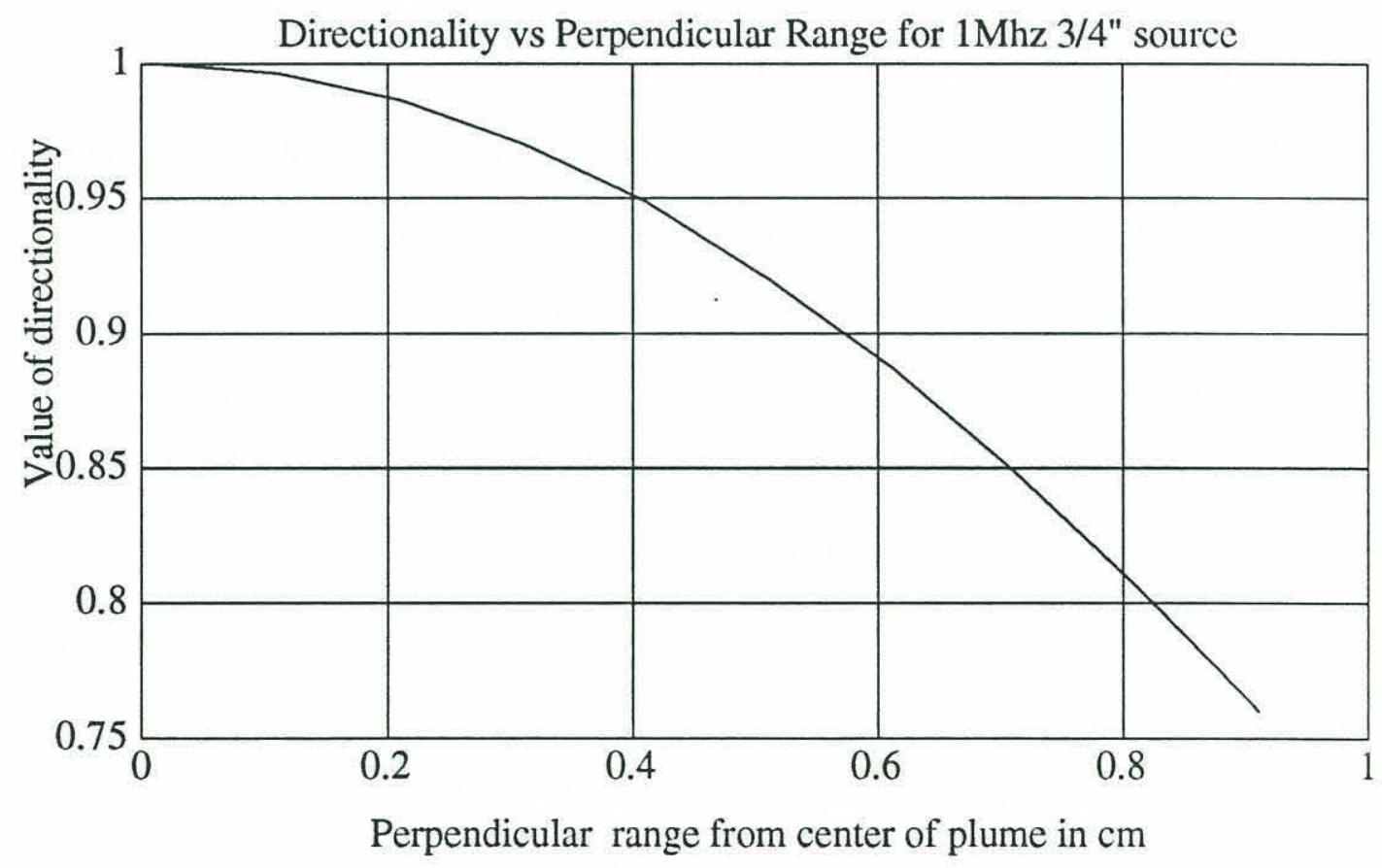

Figure 2.3

22 Clarence S. Clay and Herman Medwin, Acoustical Oceanography: Principles and Applications (New York: John Wiley \& Sons, 1977), p.155. 23 Clay, p. 454. 
This indicates that for the plume with a rough diameter less than $1 \mathrm{~cm}$ the non planer terms are small, but for the plumes beyond $2 \mathrm{~cm}$ in diameter the value falls below $(1 / 2)^{1 / 2}$. This half power value indicates strong non planer effects.

The signal generation and data acquisition system consisted of a Real Time Systems (RTS) model 4700/MR Acoustic Measurements System, using an arbitrary wave form generator (AWG). The AWG has a $64 \mathrm{~K}$ memory with a maximum frequency of $30 \mathrm{MHz}$, and 12 bit amplitude resolution. The repetition rate for the signals generated is variable from 1 microsecond up to 10 seconds in 1 microsecond steps. The signal acquisition system uses a 12 bit digitizing multiple record (MR) board with a maximum sampling frequency of $20 \mathrm{MHz}$ and signal length of up to 1024 points per wave form and store sequentially up to 2048 wave forms.

Temperature measurements of the temperature profile of the tank during the experiment used an Omega WB-AAI-B High Resolution temperature sampling board in an NEC Power Mate Portable SX with 8 channels of chromel-alumel (K) 30 AWG thermocouple wire on a plastic rod. The thermocouple probes were spaced approximately $7.6 \mathrm{~cm}$ apart up to $53.5 \mathrm{~cm}$ with the one probe placed at the $1 \mathrm{~cm}$ to get a near bottom sample. Temperature samples were taken from all eight channels at 1 second intervals. The stated accuracy of the temperature board was $0.7^{\circ} \mathrm{C}$ with a resolution of $0.02{ }^{\circ} \mathrm{C}$ for this sample rate; but all thermocouples were verified to be within $0.1^{\circ}$ in air prior to the start of the experiment.

The system was controlled by two Macintosh computers. A IIfx contained the video digitizing board and was used mostly to obtain the video data. A IIx was programmed to run the RTS system. All the processing of the data from this facility was done on a Macintosh IIvx. 


\subsection{Salt Water System}

The other laboratory system used a large tank. The tank had cylindrical ends with radii of $122 \mathrm{~cm}$ with a $122 \mathrm{~cm}$ section inserted between the cylindrical ends. The tank had a nominal depth of $152 \mathrm{~cm}$. This gave a working volume of $11.65 \mathrm{~m}^{3}$. An anodized aluminum frame place in the tank held a heater identical to the one used in the other lab. In addition, the frame held rods with Teflon transducer holders which allowed face to face transducer distances of $163 \mathrm{~cm}$. The transducers used were also Panametrics but of $0.5^{\prime \prime}$ diameter. This diameter gives a value of $\mathrm{R}_{\mathrm{c}}=8.45 \mathrm{~cm}$. using the National Standards Institute method.

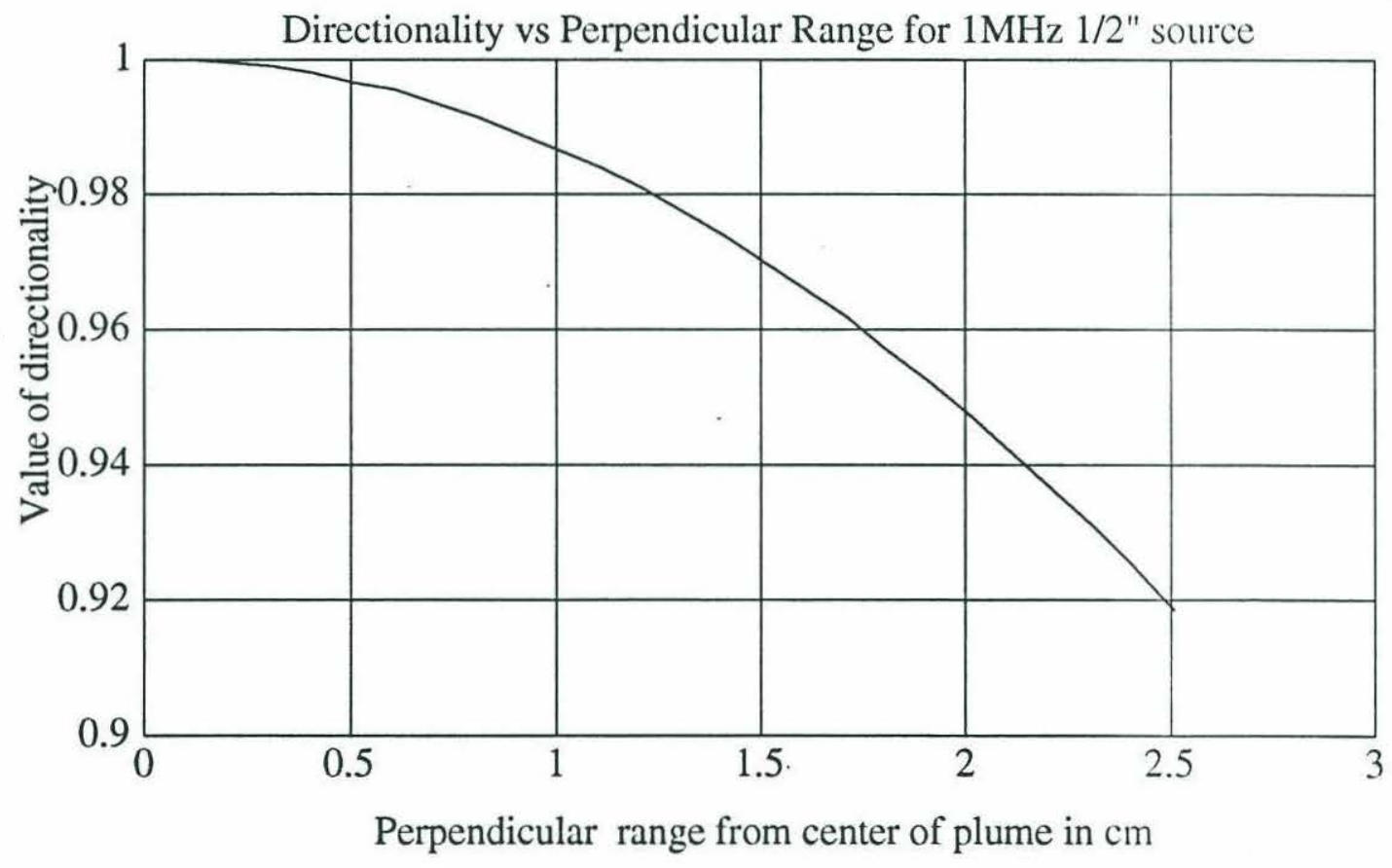

Figure 2.4

The plot of the directionality demonstrates that even for plumes greater than $5 \mathrm{~cm}$ the beam can essentially be considered a plane wave. 
The temperature sampling system used to examine the nature of the plume consisted of the same system as the other laboratory except that the array consisted of 4 groups of 2 thermocouples spaced $10 \mathrm{~cm}$ apart and placed at a height of $13 \mathrm{~cm}$ above the heater so as not to interfere with the signal transmitted at $5 \mathrm{~cm}$. The system did not give an exact qualitative assessment of the plume at the height of acoustic illumination. It did give indications of the trend in the turbulence and provided a strong indication of the variation in the plume. For other heights above the plume the array was moved up as appropriate.

The 5 volt peak to peak $995 \mathrm{kHz}$ signal for this tank was generated using an Analogic Polynomial Waveform Synthesizer Model 2020. The signal was received on a Lecroy 943010 bit $150 \mathrm{MHz}$ digital oscilloscope with a memory card using an external clock provided by an HP 8116A signal generator set at $10 \mathrm{MHz}$ to allow 256 repetitions of 200 sample points to be stored on the memory disk at $10 \mathrm{~Hz}$. The data transmission and retrieval system was controlled by a H\&H Innovative Solutions 486 computer, programmed to control the wave form synthesizer and digital oscilloscope. Data transmission from the digital oscilloscope to the local computer network was accomplished using a program called ATF developed at WHOI. The limit on the storage of wave forms to 256 was overcome by running the experiment twice for each power level of the heater to obtain approximately 50 seconds of data. 
Salt Water

Tank

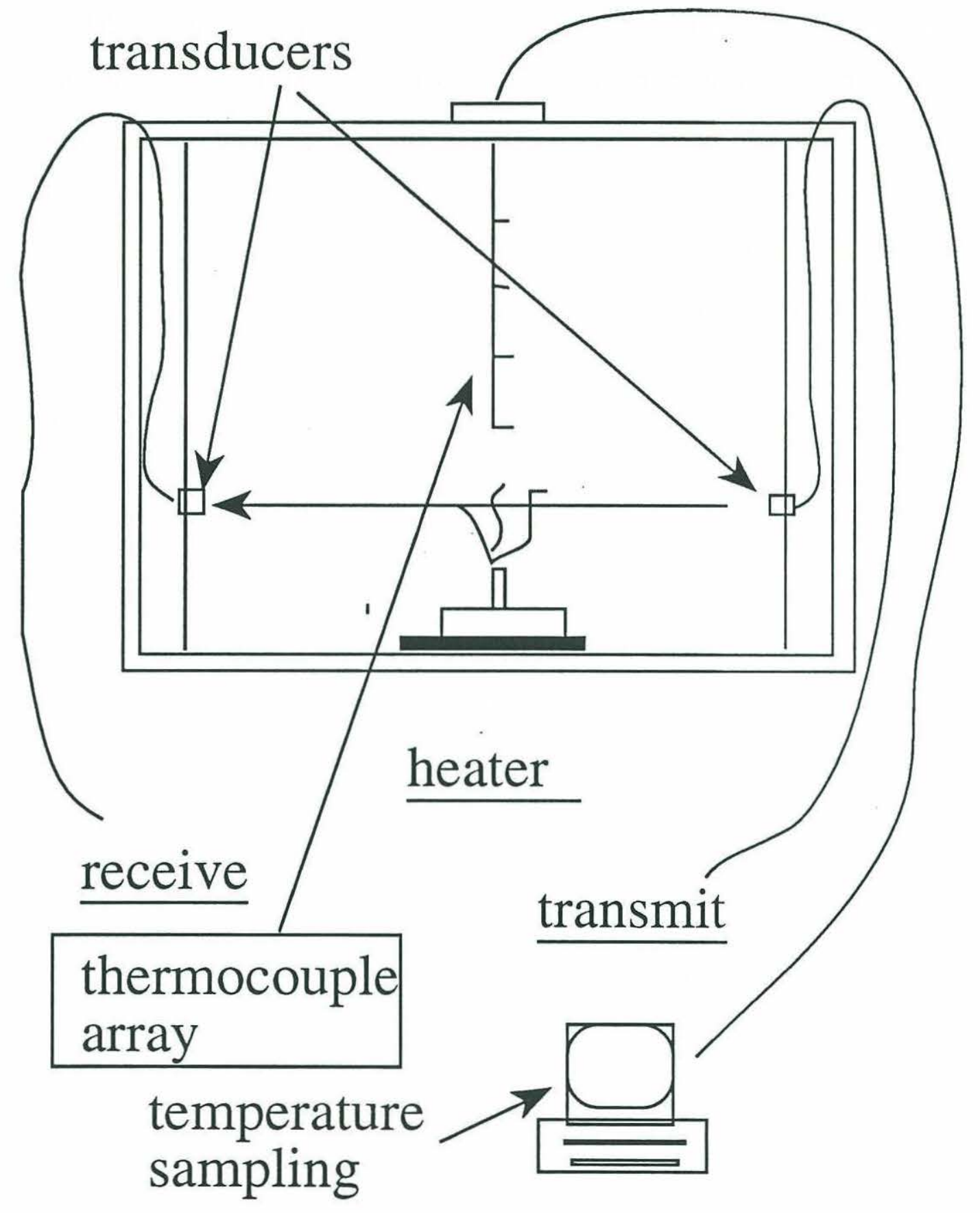

Figure 2.5 


\section{Chapter 3}

\section{Theory}

The experiment described in the previous chapter provides an easily measured result of the scattering through a plume. In order to analyze the data, several aspects of the experiment need to be discussed. The nature of the plume and the experimental requirements to ensure that the plume can be properly sampled need to be determined. Once the requirements for sampling the plume are established, the signal to be used must be produced to achieve the desired results.

After the experiment, the analysis of the data requires some approximations. The two most common approximations are the Born and the Rytov approximations. These two approximations are explored for the single iteration performance of each to assess . applicability.

Finally, a brief explanation of the scintillation index is provided. This allows a slightly different insight into the effects of the turbulence on a signal, and may assist in visualizing the interaction between the plume and the signal.

\subsection{Buoyant Plume .}

In order to properly set up the experiment, the rate of change of the phenomena being sampled needs to be determined. The actual phenomena need not be understood in order to view it. Actually, improper sampling of the phenomena can be useful if it is understood what the sampling limits are. The plume is created using a small heat source on the bottom of the tank. By heating the water around the source a narrow buoyant plume is created. The nature of the plume is determined by the power applied to the heater. At the heaters upper power limit a turbulent plume in our experimental system has a flow rate (U) 
of $\approx 2.75 \mathrm{~cm} / \mathrm{s}$. For a turbulent system the model for the turbulent flow provides an overall velocity of $U+u$, where $u$ is a random variable with a maxlul= $\alpha U$ and $\alpha<1$. The Kolmogorov microscales are defined for length $(\eta)$, time $(\tau)$, and velocity $(v)$. As such, the minimum inner (small) scale for a turbulent plume in water is given by ${ }^{24}$ equation (4); where $v$ is the kinematic viscosity $\left(1 * 10^{-6} \mathrm{~m}^{2} \mathrm{~s}^{-1}\right.$ for water at $\left.20^{\circ} \mathrm{C}\right)$, and $\varepsilon$ is the dissipation rate per unit mass $\left(\mathrm{m}^{2} \mathrm{~s}^{-3}\right)$. The expression for $\varepsilon$ is given by ${ }^{25}$ equation (5).

$$
\begin{aligned}
& \eta=\left(\frac{v^{3}}{\varepsilon}\right)^{\frac{1}{4}} \\
& \tau=\left(\frac{v}{\varepsilon}\right)^{\frac{1}{2}} \\
& v=(v \varepsilon)^{\frac{1}{4}} \\
& \varepsilon \approx \frac{u^{3}}{1}
\end{aligned}
$$

The integral (outer) scale of the plume is 1 , and $u$ is the random variable of the velocity. By using the value for the smallest possible eddy size and the velocity of the plume, with the requirement for every sample to be no more than one eddy size from the previous sample, an effective sample rate can be determined. For a maximum value of $U+\mathrm{ll}=5.5 \mathrm{~cm} / \mathrm{s}$ and $l=1 \mathrm{~cm}: \mathrm{h} \approx 88^{*} 10^{-6} \mathrm{~m}$ and a velocity of $5.5 \mathrm{~cm} / \mathrm{s}$ the sample rate is $624 \mathrm{~Hz}$ which corresponds to a time of $1.6 \mathrm{~ms}$. Initial experimental estimates of the maximum velocity ( 2 $\mathrm{cm} / \mathrm{s})$ and the minimum $1(2.5 \mathrm{~cm})$ gave a value for $\mathrm{h} \approx 236^{*} 10^{-6} \mathrm{~m}$ and a value for the sample rate at $84.6 \mathrm{~Hz}$. The experiment was designed using this initial value of a sampling frequency. Subsequent experimentation using a sample rate of $100 \mathrm{~Hz}$ lead to the discovery that variations in the plume were limited to less than $2.5 \mathrm{~Hz}$. This indicates a

$24 \mathrm{H}$. Tennekes and J.L. Lumley, A First Course in Turbulence (Cambridge: The MIT Press, 1972), p.20.

25 Tennekes, p.20. 
value of $u \approx 2.3 \mathrm{~mm} / \mathrm{s}$ for a value of 1 of $1.0 \mathrm{~cm}$ and corresponding values of $\eta=8 \mathrm{~mm}$ (using the same method as above and $2 \mathrm{~cm}$ ), $\tau=.91 \mathrm{~s}$ and $v=1.1 \mathrm{~mm} / \mathrm{s}$.

Nothing else is necessary to establish the criteria for sampling the plume created. Since the plume is initially laminar and eventually becomes turbulent, the limiting values for the experiment are outlined above.

The structure of the plume itself as it becomes turbulent is of interest in order to investigate the trends in the received acoustical data. From Batchelor ${ }^{26}$ the weight deficiency produced per unit time at the source $\left(\rho_{0} \mathrm{~F}\right)$ for a laminar plume is given by equation (6).

$$
F=\int_{0}^{\infty} U g\left(\frac{T(r)-T_{0}}{T_{0}}\right) 2 \pi r d r
$$

Here $g$ is the acceleration due to gravity, $\mathrm{T}_{0}$ is the absolute temperature of the field, and $\mathrm{T}(\mathrm{r})$ is the absolute temperature of the plume. $\mathrm{T}(\mathrm{r})$ is a function of $\mathrm{r}$; the axial distance from the center of the plume. This value $(\mathrm{F})$ is conserved for a given height $\mathrm{z}$. A Gaussian temperature distribution is given by equations (7) and (8).

$$
\begin{aligned}
& T(r)-T_{0}=\Delta T e^{\frac{r^{2}}{b^{2}}}=\Delta T(r) \\
& F=\frac{\pi U g \Delta T}{T_{0}}
\end{aligned}
$$

Once the plume becomes turbulent the mean temperature distribution continues to remain Gaussian. ${ }^{27}$ Batchelor provides equations to use for the average $\mathrm{T}(\mathrm{r})-\mathrm{T}_{0}$ and mean velocity $(\bar{U})$ for a buoyant plume in air in equations (9) and (10).

$$
\overline{\mathrm{U}}=4.7 \frac{\mathrm{F}^{1 / 3}}{z^{1 / 3}} \mathrm{e}^{-96 \mathrm{r}^{2} / \mathrm{z}^{2}}
$$

26 G.K. Batchelor, "Heat Convection and Bouyancy Effects in Fluids, "Quarterly Journal of the Royal Meterological Society,80 (1954), pp. 339-358.

27 Batchelor, p.344. 


$$
\overline{T(r)-T_{0}}=11.0 \frac{T_{0}}{g} \frac{F^{2 / 3}}{z^{5 / 3}} e^{-71 r^{2} / z^{2}}
$$

An example using $\mathrm{U}=2 \mathrm{~cm} / \mathrm{s}$ and a $\Delta \mathrm{T}$ near the origin of $10^{\circ} \mathrm{C}$ is shown in figure 3.1 :

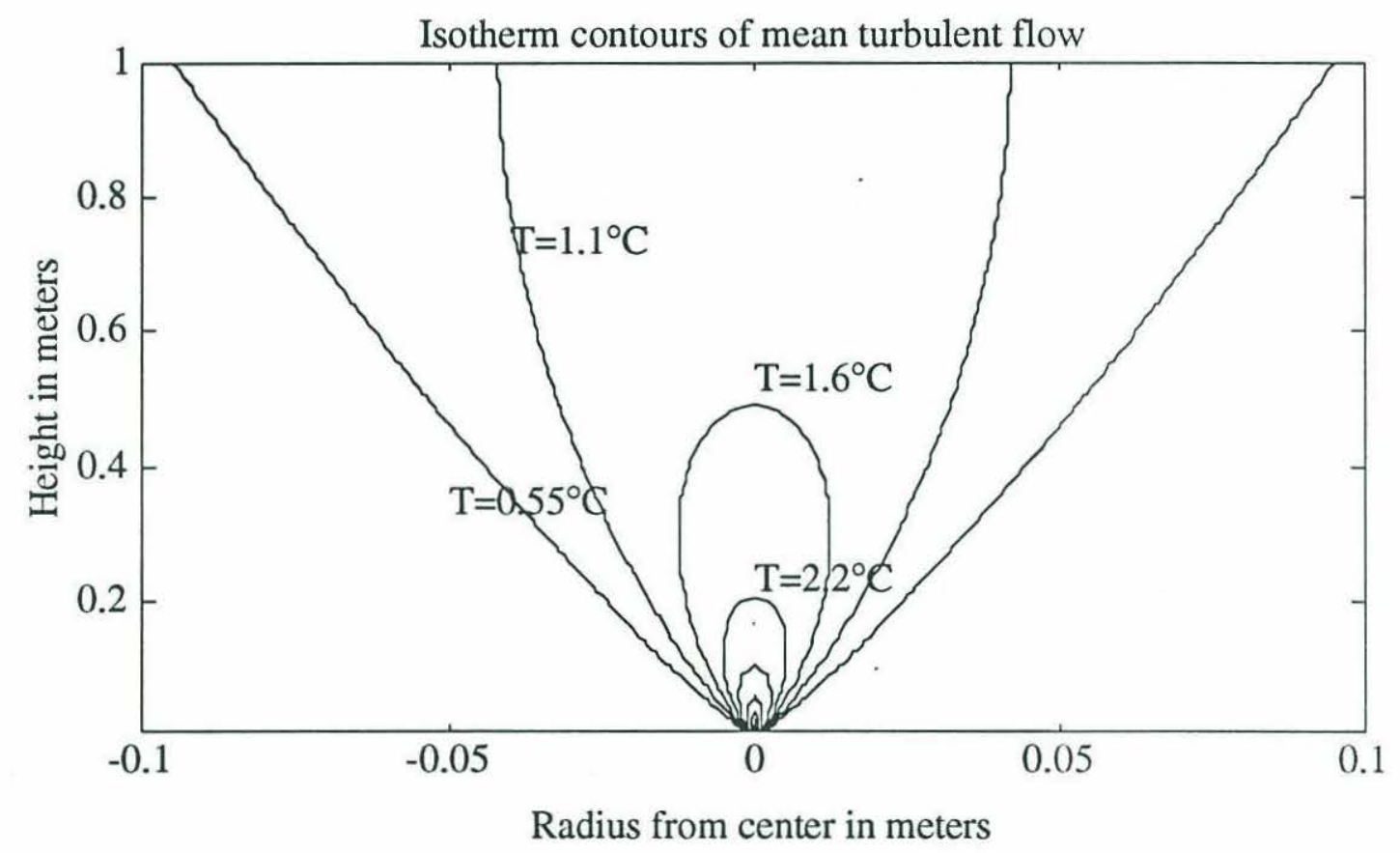

Figure 3.1

One of the most significant features of the plot is the $-5 / 3$ exponential decay of the peak temperature as the height increases. This is opposed to a -1 decay noted for a laminar plume. This decay indicates that as the height above the source increases the scintillation index will decrease at a lesser rate than the $-5 / 3$, because, the peak values of the index of refraction will decrease at a lesser rate due to spreading of the plume. In addition the broadening of the plume indicates a broadening of the eddies and demonstrates that the peak modulation frequency of the received signal should decrease at a rate corresponding to a broadening rate of the plume. 


\subsection{Signal Description}

The initial genesis of the problem being investigated was a telemetry question involving the possibility of discrete turbulent interference of shallow water signals. The nature of this interference would be discrete phenomena, such as fresh water springs or heat vents. The common effect of both sources of interference is the variation in the index of refraction within the plume by variations in salinity or temperature. Many telemetry devices involve the use of signals in the 10's of $\mathrm{kHz}$ range. An initial value of $10 \mathrm{kHz}$ for a signal was used for modeling. The natural phenomena discussed above are in general meter scale sources.

A common method for evaluating scattering involves the factor $\mathrm{ka}$ : $\mathrm{k}$ is the wave number $w / c(w=2 * \pi * f, c=$ speed of sound in water) and ' $a$ ' is an effective radius of the scatterer. For a $10 \mathrm{kHz}$ signal, $\mathrm{c}=1500 \mathrm{~m} / \mathrm{s}$ and an 'a' $=1 \mathrm{~m}$ ka becomes $\approx 42$; which scales the frequency to $1 \mathrm{MHz}$ for this heater with a $1 \mathrm{~cm}$ plume.

To allow easy processing at the desired frequency a continuous wave (CW) signal was chosen. The length of the signal is limited by the necessary sampling rate and the avoidance of backscattered echoes off of the tank walls. A $100 \mathrm{~Hz}$ sampling rate limits the total length of the signal to $10 \mathrm{~ms}$. To avoid echoes in the reception of the signal the signal length should be less than the transit time; the transit time for the small tank is approximately $0.2 \mathrm{~ms}$ (transducer to back wall and back to transducer). To allow a large tolerance factor for time filtering the echo signals a factor of 20 reduces the limit to $0.01 \mathrm{~ms}$ or 10 cycles at $1 \mathrm{MHz}$. Ten cycles still provides a strong $1 \mathrm{Mhz}$ signal and allows about 10 samples per cycle with a sampling rate of $10 \mathrm{MHz}$.

The signal in Figure 3.2 is the raw received signal for the experiment. In order to determine the magnitude and phase of the signal most accurately, a block FFT (i.e. no windowing except the basic rectangular window) is performed and the result is shown in Figure 3.3. A rectangular window provides an accurate assessment of the frequency 
amplitude with one frequency present with a processing gain of $1.0^{28}$.

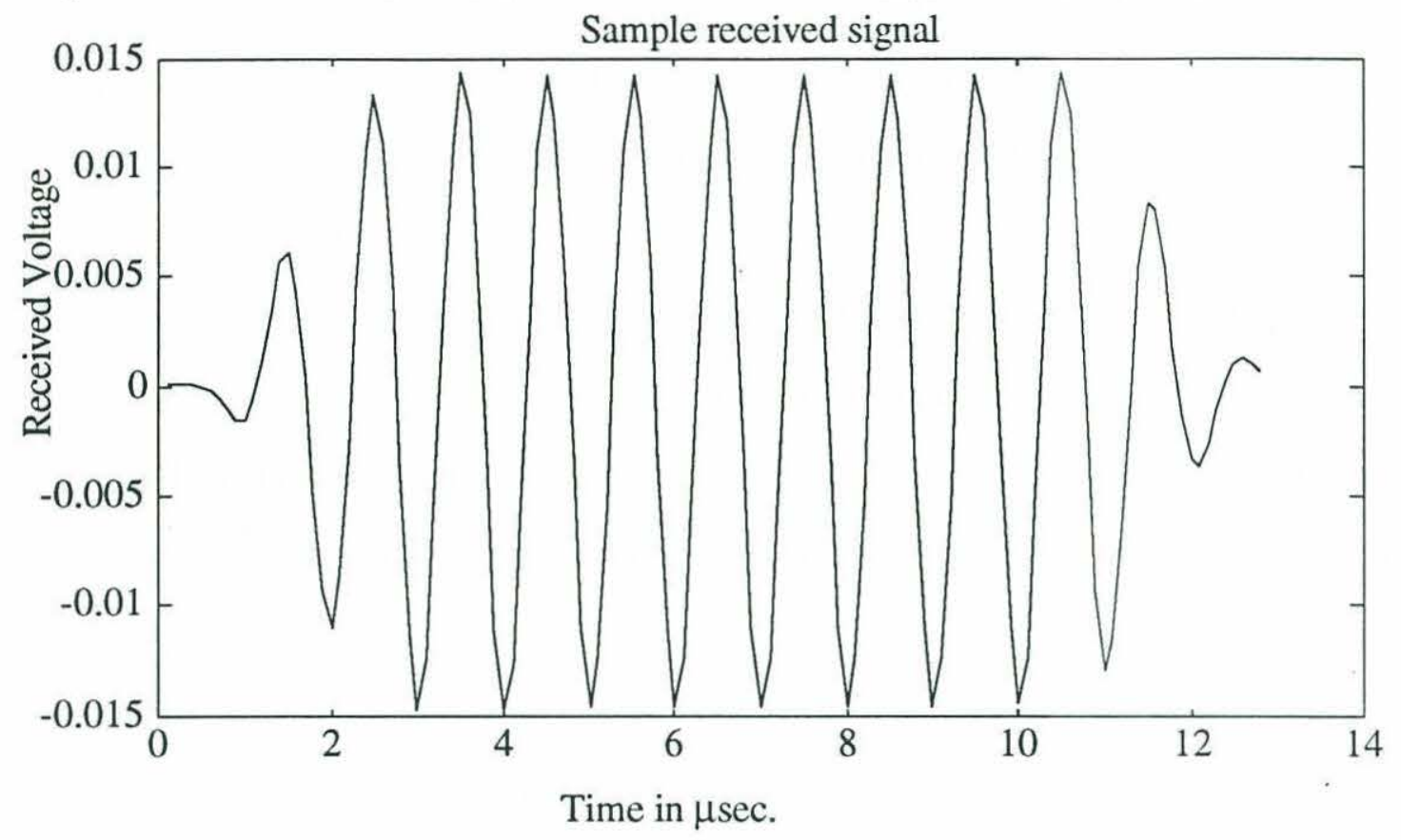

Figure 3.2

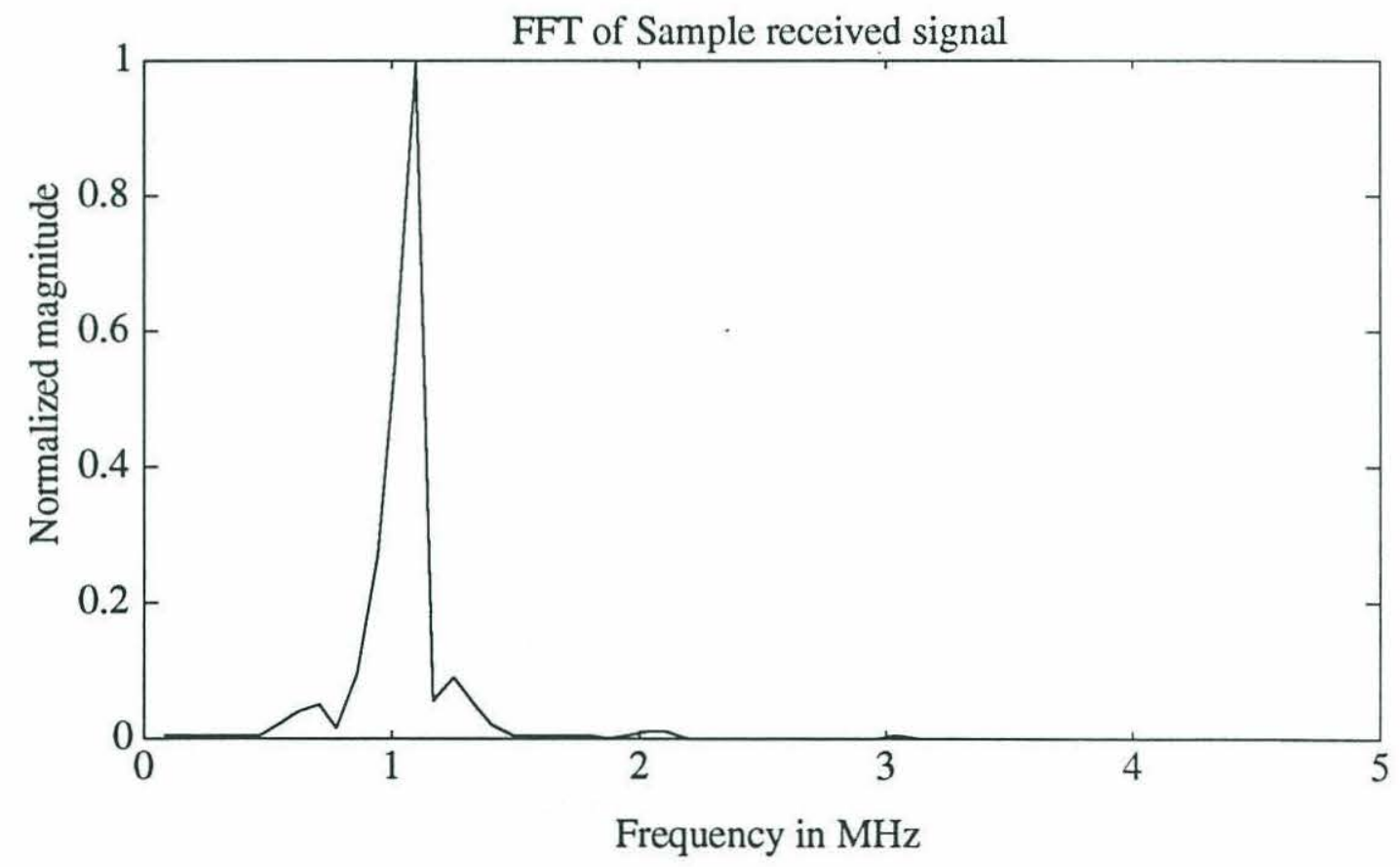

Figure 3.3

\footnotetext{
${ }^{28}$ Fredrick J. Harris, "On the Use of Windows for Harmonic Analysis with the Discrete Fourier Transform, "Proceedings of the IEEE, 66, No.1 (January 1978), 54 .
} 


\subsection{Interaction of Signal and Plume}

The image of the plume given by the acoustic scattering through the plume is really a measure of a slice of the plume. Since the signal propagation path is directly through the plume the resulting signal is the result of the average index of refraction across the plume.

\subsubsection{Wave Equation}

Starting with the wave equation from Ingard and Morse, ${ }^{29}$ and following the applicable portions of the derivation in Oeschger, ${ }^{30}$ an analytic form for the forward scattered signal is obtained.

$$
\begin{aligned}
& \left(\nabla^{2}-\frac{1}{c^{2}} \frac{\partial}{\partial t^{2}}\right) P(r, t)= \\
& \left(\frac{1}{c^{2}} \gamma_{k}(r, t) \frac{\partial}{\partial t^{2}}+\underline{\nabla} \cdot\left(\gamma_{\rho}(r, t) \underline{\nabla}\right)+\frac{1}{c^{2}} \frac{\partial u}{\partial t} \cdot \underline{\nabla}+\frac{2}{c^{2}} u \cdot \underline{\nabla} \frac{\partial}{\partial t}\right) P(r, t)
\end{aligned}
$$

$P(r, t)$ is the pressure at location $\mathbf{r}$ and time $t ; c$ is the ambient sound speed;

$\gamma_{\kappa}=\frac{\kappa-\kappa_{0}}{\kappa_{0}}$ is the relative compressibility; $\kappa_{0}$ is the ambient compressibility;

$\gamma_{\rho}=\frac{\rho-\rho_{0}}{\rho_{0}}$ is the relative density; $\rho_{0}$ is the ambient density; and $u$ is the fluid velocity.

Since the value for $\tau$ as calculated above indicates that the variability of the

inhomogeneities is many times slower than the period of the sound wave, the time dependence will be ignored for $\gamma_{\kappa}$ and $\gamma_{\rho}$. In addition, the direction of $\mathrm{u}$ is essentially

perpendicular to the direction of the sound wave propagation; this eliminates the last two terms in the parenthesis of (11). Taking the Fourier transform

29 Philip M. Morse and K. Uno Ingard, Theoretical Acoustics (Princeton, New Jersey: Princeton University Press, 1968), pp. 407-409.

30 Oeschger, pp. 12-15. 
$\left(p(r, w)=\int P(r, t) e^{i w t} d t\right.$ and, $\left.P(r, t)=\frac{1}{2 \pi} \int p(r, w) e^{-i w t} d w\right)$ leaves equation (12), which is equation 8.1.12 from Morse and Ingard. ${ }^{31}$

$$
\left(\nabla^{2}+k^{2}\right) p(r, w)=\left(-k^{2} \gamma_{k}(r)+\underline{\nabla} \cdot\left(\gamma_{\rho}(r) \underline{\nabla}\right)\right) p(r, w)
$$

The scattered pressure is determined by the integral solution to (12), which, for the boundary free case, is given in Morse and Ingard as equation 8.1.1332.

$$
\begin{aligned}
& \mathrm{p}_{\mathrm{s}}(r, \mathrm{w})= \\
& \int\left\{\mathrm{k}^{2} \gamma_{\mathrm{x}}\left(\boldsymbol{r}^{\prime}\right) \mathrm{p}\left(\boldsymbol{r}^{\prime}, \mathrm{w}\right) \mathrm{g}\left(\boldsymbol{r} \mid \mathbf{r}^{\prime}\right)+\gamma_{\rho}\left(\boldsymbol{r}^{\prime}\right) \underline{\nabla p}\left(\boldsymbol{r}^{\prime}, \mathrm{w}\right) \cdot \underline{\nabla g}\left(r \mid r^{\prime}\right)\right\} \mathrm{d} \boldsymbol{r}^{\prime}
\end{aligned}
$$

The relative compressibility and the relative density are the two primary sources of variation in the effective index of refraction in fluid plume. The relative importance of these two factors can be determined by considering the variation in temperature in the plume. Using a value of $5{ }^{\circ} \mathrm{C}$ for the change across the plume; the values for the change in density and sound speed for this variation can be found. ${ }^{33}$ The sound speed at $20{ }^{\circ} \mathrm{C}$ is 1482.3 and at $25^{\circ} \mathrm{C}$ it is 1497.5 . The value for the density of fresh water at $20{ }^{\circ} \mathrm{C}$ is 998.213 and at $25^{\circ} \mathrm{C}$ is 997.053 .

$$
\begin{aligned}
& \gamma_{x}=\frac{\rho_{0} c_{\circ}^{2}-\rho c^{2}}{\rho c^{2}}=-0.019 \\
& \gamma_{\rho}=\frac{\rho_{0}-\rho}{\rho}=-0.0012
\end{aligned}
$$

The ratio between $\gamma_{\mathrm{K}}$ and $\gamma_{\rho}$ is about 15 . The scattering due to changes in the compressibility is far more significant than that due to variations in the relative density.

\footnotetext{
${ }^{31}$ Morse, p. 410 .

32 Morse, p. 410 .

33 L.E. Kinsler et al.,Fundamentals of Acoustics (New York: Wiley and Sons, 1982), p. 107.
} 
An expression for $\gamma_{\kappa}$ in terms of temperature in accordance with Oeschger $^{-34}$ can be found by assuming that $\rho \approx \rho_{0}$ and using a linear approximation of the sound speed such that $\mathrm{c} \cong \frac{\mathrm{dc}}{\mathrm{dT}} \Delta \mathrm{T}+\mathrm{c}_{0}$. Since $\mathrm{c} \approx \mathrm{c}_{0}$, the expression for $\gamma_{\kappa}$ becomes equation (15) which reduces to equation (16).

$$
\begin{aligned}
& \gamma_{\kappa}=\frac{c_{0}^{2}-\left(\frac{d c}{d T} \Delta T+c_{0}\right)^{2}}{c_{0}^{2}} \\
& \gamma_{\kappa}=\frac{-2}{c_{0}} \frac{d c}{d T} \Delta T(r) .
\end{aligned}
$$

Using these approximations, the scattered equation becomes equation (17), where $\alpha=\frac{1}{c_{0}} \frac{d c}{d T}$.

$$
\mathrm{p}_{\mathrm{s}}(r, \mathrm{w})=-2 \alpha \mathrm{k}^{2} \int \Delta \mathrm{T}\left(\mathrm{r}^{\prime}\right) \mathrm{p}\left(\mathrm{r}^{\prime}, \mathrm{w}\right) \mathrm{g}\left(r \mid \mathrm{r}^{\prime}\right) \mathrm{d} \mathrm{r}^{\prime}
$$

The generalized asymptotic form of the Greens function for one, two and three dimensions from Oeschger ${ }^{35}$ reduces to equation (18) where $d$ is the dimensionality, and $|r|$ is the radial coordinate in three and two dimensions or the linear coordinate of the onedimensional case.

$g_{d}\left(r \mid r^{\prime}\right)=(\pi|r|)^{\frac{1-d}{2}} 2^{-\left(\frac{1+d}{2}\right)} k^{\left(\frac{d-3}{2}\right)} \exp \left(i\left(k|r|-k_{*} \cdot r^{\prime}+\pi\left(\frac{3-d}{4}\right)\right)\right)$

${ }^{34}$ Oeschger, p. 14.

35 Oeschger, p.15. 


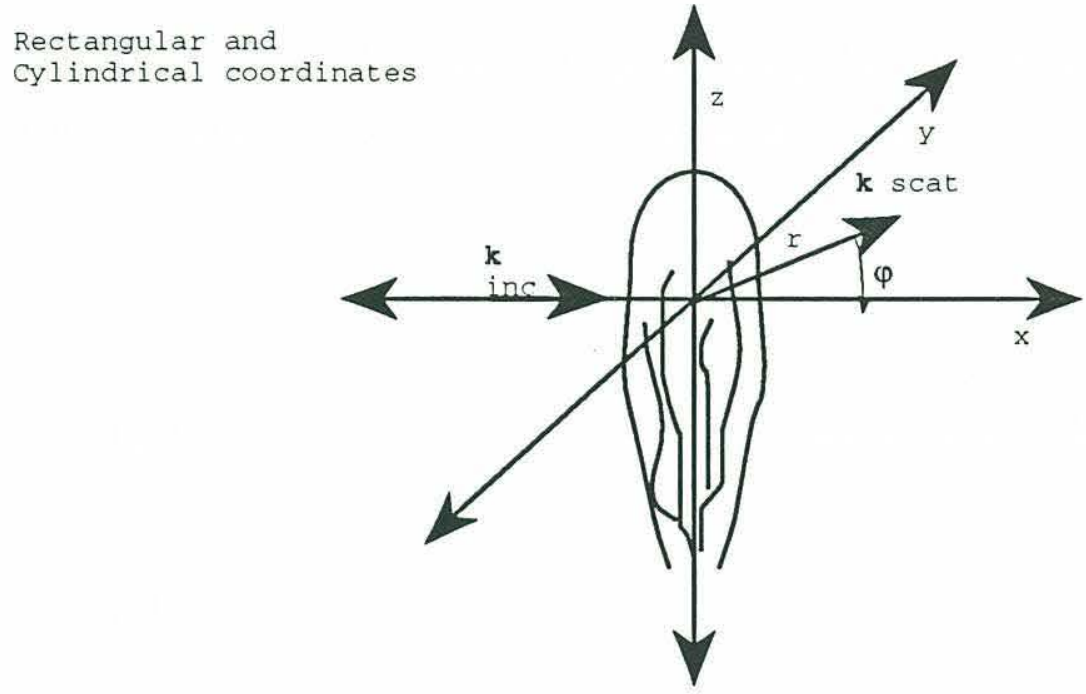

Figure 3.4

\subsubsection{Born Approximation}

In order to obtain a solution, a single iteration Born approximation will be used. In accordance with Ishimaru ${ }^{36}$, in order to use the Born approximation successfully: $\left(\mathrm{n}^{2}-\right.$ 1) $\mathrm{kD}<<1$. This is satisfied for a plume up to $1.2 \mathrm{~cm}$ in diameter. The Born approximation allows the substitution inside the integral for $\mathrm{p}_{\mathrm{s}}$ of $\mathrm{p}_{\mathrm{i}}(\mathrm{w}) \exp \left(\mathrm{i}_{\mathrm{i}} \cdot r^{\prime}\right)$ for $\mathrm{p}\left(\mathrm{r}^{\prime}, w\right)$. Upon making this substitution the scattered field becomes equation (19).

$$
\begin{gathered}
\mathrm{p}_{\mathrm{s}}(r, \mathrm{w})=\alpha(2 \pi r)^{\frac{-1}{2}} \mathrm{k}^{\left(\frac{3}{2}\right)} \mathrm{p}_{\mathrm{i}}(\mathrm{w}) \exp \left(\mathrm{i}\left(\mathrm{kr}+\pi\left(\frac{5}{4}\right)\right)\right) \\
\mathrm{X} \int \Delta \mathrm{T}\left(\mathrm{r}^{\prime}\right) \mathrm{e}^{-\mathrm{i}\left(\mathrm{k}_{\mathrm{o}}-\mathrm{k}_{1}\right) \cdot \mathbf{r}^{\prime}} \mathrm{d} \mathbf{r}^{\prime}
\end{gathered}
$$

The integral portion, for $\mathbf{k}_{\mathbf{s}}=\mathbf{k}_{\mathbf{i}}$, reduces to $\int \Delta \mathrm{T}\left(\boldsymbol{r}^{\prime}\right) \mathrm{d} \boldsymbol{r}^{\prime}$. Equation (20) is in cylindrical coordinates for a two dimensional system with $\Delta T\left(r^{\prime}\right)=\Delta T e^{-\frac{r^{2}}{b^{2}}}$.

36 Ishimaru, p.22. 


$$
\mathrm{p}_{\mathrm{s}}(r, \mathrm{w})=\alpha \Delta \mathrm{Tb}^{2}\left(\frac{\pi}{2 r}\right)^{\frac{1}{2}} \mathrm{k}^{\left(\frac{3}{2}\right)} \mathrm{p}_{\mathrm{i}}(\mathrm{w}) \exp \left(i\left(k r+\pi\left(\frac{5}{4}\right)\right)\right) .
$$

Equation (20) in terms of the total field $\mathrm{p}_{t}=\mathrm{p}_{\mathrm{d}}+\mathrm{p}_{\mathrm{s}}$ is shown in figure 3.5 where $\mathrm{p}_{\mathrm{d}}$ is the direct unscattered field.
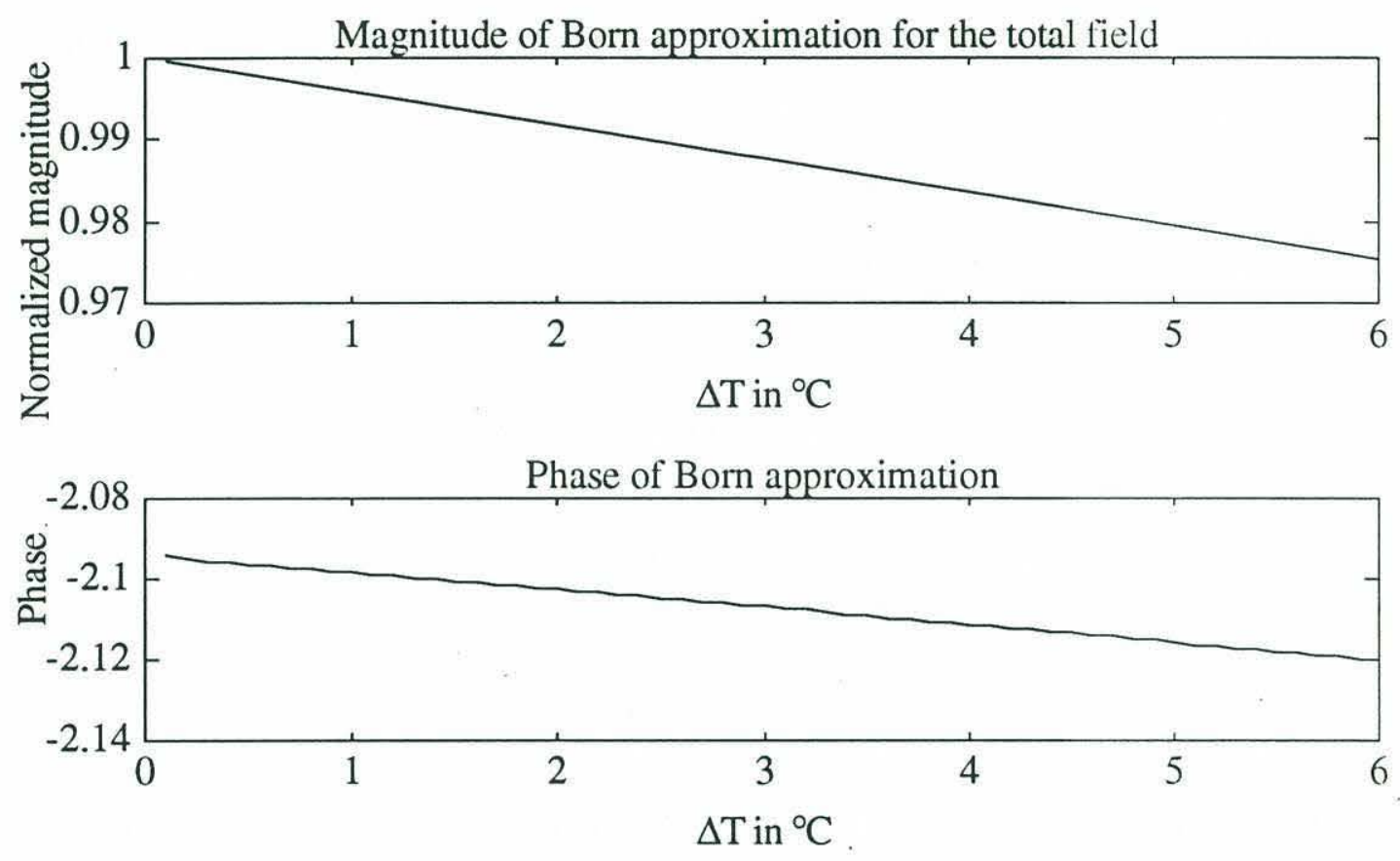

Figure 3.5

\subsubsection{Rytov Approximation}

Some ${ }^{37}$ recommend the Rytov method for solving in the forward scattered direction. This method involves the substitution of $\mathrm{p}(\mathrm{r}, \mathrm{w})=\mathrm{p}_{\mathrm{i}}(\mathrm{w}) \exp (\mathrm{i} \psi(\mathbf{r}))$; where $\psi(\mathbf{r})=\mathrm{S}(\mathbf{r})-\mathrm{i}^{*} \log \left(\mathrm{p}(\mathbf{r}) / \mathrm{p}_{\mathrm{i}}\right)$. Equation (21) is equation (12) for the case of $\gamma_{\mathrm{k}}>\gamma_{\mathrm{p}}$.

$$
\left(\nabla^{2}+k^{2}\right) p(r, w)=-k^{2} \gamma_{k}(r) p(r, w)
$$

37 Ishimaru, p. 349. 
Substituting $p\left(r^{\prime}, w\right)=p_{i}(w) \exp \left(i \psi\left(r^{\prime}\right)\right)$ and then following the substitutions for the derivation of the Rytov approximation in Ishimaru ${ }^{38}$ a solution results. Using $\psi=\psi_{0}+\psi_{1}$ with $\psi_{0}$ from $\gamma_{\mathrm{k}}=0$ the form becomes equation (22).

$$
\left(\nabla^{2}+k^{2}\right) p_{0}(r, w) \psi_{1}=-\left(\nabla \psi_{1} \cdot \nabla \psi_{1}+k^{2} \gamma_{k}\right) p_{0}(r, w)
$$

Equation (22) is in the form of the inhomogeneous wave equation which when converted to integral form for $\psi_{1}$ becomes equation (23).

$$
\psi_{1}(r, w)=\frac{1}{p_{0}(r, w)} \int\left(\nabla \psi_{1} \cdot \nabla \psi_{1}+k^{2} \gamma_{k}\right) p_{0}\left(r^{\prime}, w\right) g\left(r \mid r^{\prime}\right) d r^{\prime}
$$

Equation (23) yields equation (24) for a first iteration of $\psi_{1}=0$ on the right hand side.

$$
\psi_{1}(r, w)=\frac{1}{p_{0}(r, w)} \int k^{2} \gamma_{k} p_{0}\left(r^{\prime}, w\right) g\left(r \mid r^{\prime}\right) d r^{\prime}
$$

Since $\mathrm{p}_{0}$ is defined for $\gamma_{\mathrm{x}}=0$ then $\Delta \mathrm{T}(\boldsymbol{r})=0$ for all $\mathbf{r}$, the variations in $\mathrm{p}_{0}$ are due only to propagation in the media. The distance across the plume is only about $2.5 \mathrm{~cm}$ and the decay from $r=25 \mathrm{~cm}$ to $\mathrm{r}=50 \mathrm{~cm}$ in the signal is only $4 \%$. Using $p_{1}$ for the magnitude of $p_{0}(r, w)$ and $p_{0}\left(r^{\prime}, w\right)$ is not a bad approximation.

$$
\begin{aligned}
& \Psi_{0}(r)=S(r)-i \log \left(p(r) / p_{i}\right) \\
& S(r)=k_{i} \cdot r-k_{0} \\
& \Psi_{10}(r)=e^{-i k_{i} \cdot r} \int e^{i k_{1} \cdot r^{\prime}} g\left(r \mid r^{\prime}\right) k^{2} \gamma_{k} d r^{\prime}
\end{aligned}
$$

For $\gamma_{\mathrm{x}}=-2 \alpha \Delta \mathrm{T}\left(\boldsymbol{r}^{\prime}\right)$ and the Green's function as defined in (18), equation (25) arrives at equation (26).

${ }^{38}$ Ishimaru, p. 350. 


$$
\psi_{10}\left(r^{\prime}\right)=\alpha\left(2 \pi\left|r^{\prime}\right|\right)^{\frac{1-d}{2}} k^{\frac{d+1}{2}} e^{i \pi\left(\frac{7-d}{4}\right)} e^{i\left(k\left|r^{\prime}\right|-k_{1} \cdot r^{\prime}\right)} \int \Delta T\left(r^{\prime \prime}\right) d r^{\prime \prime}
$$

Equation (27) is equation (26) in cylindrical coordinates.

$$
\psi_{10}\left(r^{\prime}\right)=\alpha \Delta \mathrm{Tb}^{2}\left(\frac{\pi}{2\left|r^{\prime}\right|}\right)^{\frac{1}{2}} k^{\frac{3}{2}} e^{i \pi\left(\frac{5}{4}\right)} e^{i k\left|r^{\prime}\right|(1-\cos (\varphi))}
$$

Using $\Delta \mathrm{T}\left(\mathrm{r}^{\prime}\right)=\Delta \mathrm{T} \mathrm{e}^{-\frac{\mathrm{r}^{2}}{\mathrm{~b}^{2}}}$ the result becomes equation (28).

$$
\psi_{10}\left(r^{\prime}\right)=\alpha \Delta \operatorname{Tb}^{2}\left(\frac{\pi}{2\left|r^{\prime}\right|}\right)^{\frac{1}{2}} k^{\frac{3}{2}} e^{i \pi\left(\frac{5}{4}\right)} e^{i k\left|r^{\prime}\right|(1-\cos (\varphi))}
$$

Directly inserting this into $\mathrm{p}_{\mathrm{s}}(\mathbf{r})=\mathrm{p}_{\mathrm{i}}(\mathrm{w}) \mathrm{e}^{\Psi 10}$ gives a similar solution to the Born approximation but with slightly lower slope and an opposite phase variation. The total received signal $\mathrm{p}_{\mathrm{t}}$ is shown in figure 3.6.

If the asymptotic solution for the Green's function given in (15) cannot be used for the determination of $\psi_{10}(\mathbf{r})$, then for the axisymmetric case the Green's function is given by equation (29), and the expression becomes equation (30).

$$
\begin{aligned}
& g\left(r \mid r^{\prime}\right)=\frac{1}{4} H_{0}^{(1)}\left(k\left|r-r^{\prime}\right|\right) . \\
& \psi_{10}(r)=\frac{-2 \alpha k^{2}}{4} e^{-i k_{i} \cdot r} \int \Delta T^{\prime}\left(r^{\prime}\right) e^{i k_{1} \cdot r^{\prime}} H_{0}^{(1)}\left(k\left|r-r^{\prime}\right|\right) d r^{\prime} .
\end{aligned}
$$

This function has a strong pole at the points where $\mathbf{r}=\mathbf{r}^{\prime}$. To take the Rytov approximation to the next approximation beyond $\psi_{10}$ to $\psi_{110}$ insert $\psi_{10}$ as shown in equation (31).

$\psi_{110}(r)=\psi_{10}(r)+\frac{1}{p(r, w)} \int G\left(r-r^{\prime}\right) \nabla \psi_{10}\left(r^{\prime}\right) \cdot \nabla \psi_{10}\left(r^{\prime}\right) p\left(r^{\prime}, w\right) d r^{\prime}$ 

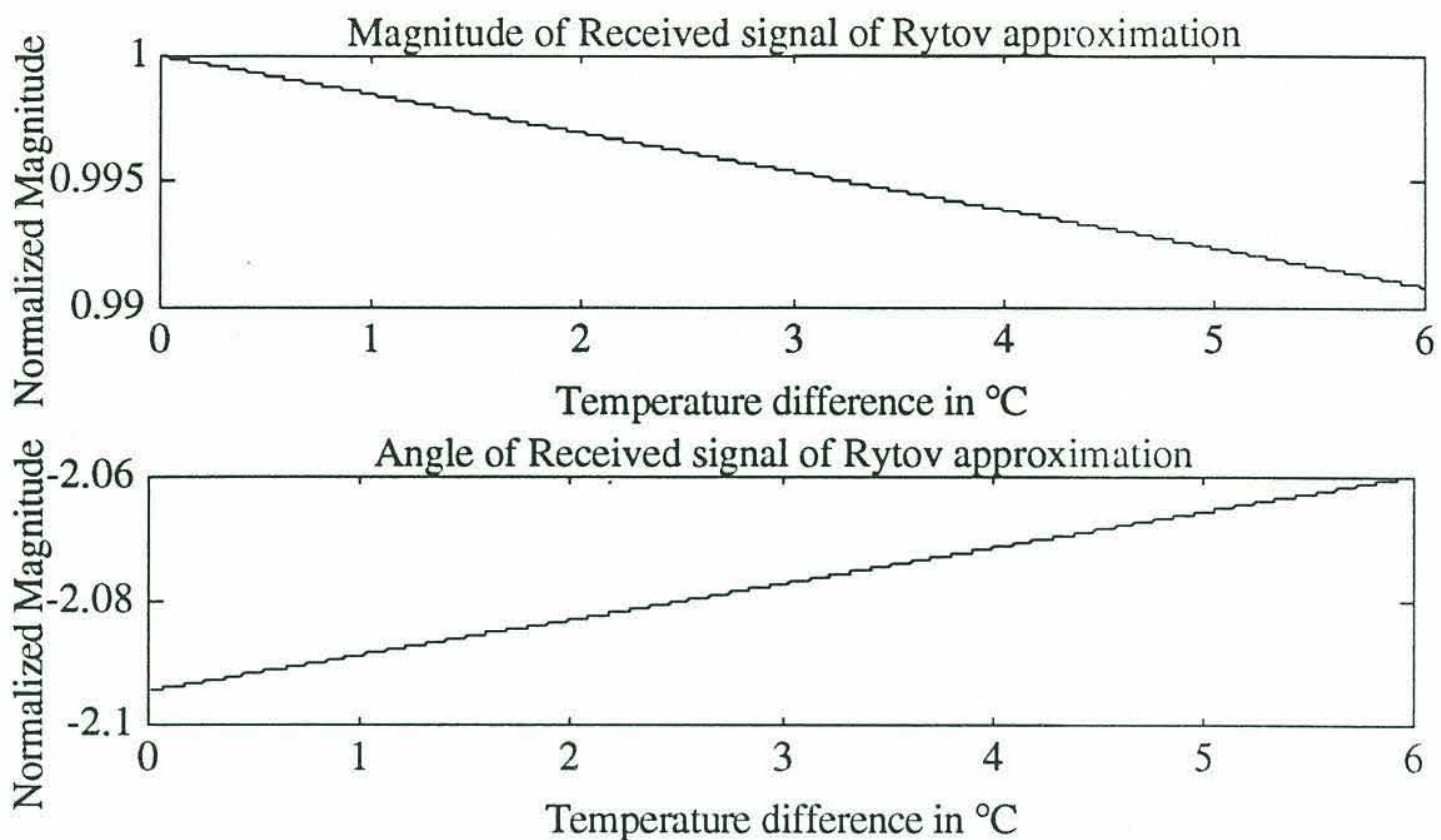

Figure 3.6

\subsection{Cylindrical Approximation}

In order to make the comparison between different power levels of the plume, the plume will be modeled as a fluid cylinder of constant density. From Stanton ${ }^{39}$ the value for the scattered pressure field is given by equations (32) and (33).

$$
\begin{aligned}
& \mathrm{p}_{i n c}=\mathrm{P}_{0} \sum_{\mathrm{m}=0}^{\infty} \varepsilon_{\mathrm{m}} i^{\mathrm{m}} \cos (\mathrm{m} \phi) J_{m}(\mathrm{kr}) \\
& \mathrm{p}_{i n t}=\mathrm{P}_{0} \sum_{\mathrm{m}=0}^{\infty} \mathrm{A}_{\mathrm{m}} \cos (\mathrm{m} \phi) J_{m}\left(\mathrm{k}^{*} r\right) \\
& \mathrm{p}_{\mathrm{s}}=\mathrm{P}_{0} \sum_{\mathrm{m}=0}^{\infty} \mathrm{B}_{\mathrm{m}} \cos (\mathrm{m} \phi) H_{\mathrm{m}}^{(1)}(\mathrm{kr})
\end{aligned}
$$

39 T. K. Stanton, "Sound Scattering by Cylinders of Finite Length.I. Fluid Cylinders," The Journal of the Acoustical Society of America,83, No.1 (January 1988), 55-63. 
The Neumann numbers are $\varepsilon_{0=1}=1$ and $\varepsilon_{\mathrm{m}}=2$ for all other $\mathrm{m}$; and $\mathrm{k}^{*}$ is the wave number inside the cylinder.

$$
\begin{gathered}
\mathrm{B}_{\mathrm{m}}=-\varepsilon_{\mathrm{m}} i^{m /\left(1+i \mathrm{C}_{\mathrm{m}}\right)} \\
\mathrm{C}_{\mathrm{m}}=\frac{\left[J_{\mathrm{m}}^{\prime}\left(\mathrm{k}^{*} \mathrm{a}\right) N_{\mathrm{m}}(\mathrm{ka})\right] /\left[J_{\mathrm{m}}\left(\mathrm{k}^{*} \mathrm{a}\right) J_{\mathrm{m}}^{\prime}(\mathrm{ka})\right]-g h\left[N_{\mathrm{m}}{ }^{\prime}(\mathrm{ka}) / J_{\mathrm{m}}^{\prime}(\mathrm{ka})\right]}{\left[J_{\mathrm{m}}^{\prime}\left(\mathrm{k}^{*} \mathrm{a}\right) J_{\mathrm{m}}(\mathrm{ka})\right] /\left[J_{\mathrm{m}}\left(\mathrm{k}^{*} \mathrm{a}\right) J_{\mathrm{m}}^{\prime}(\mathrm{ka})\right]-g h} \\
g=\frac{\rho^{*}}{\rho}, \quad \mathrm{h}=\frac{\mathrm{C}^{*}}{\mathrm{c}}
\end{gathered}
$$

The response of the scattered field in the forward direction for the cylinder model divided by $\mathrm{P}_{0}$ is shown in figure 3.7 .
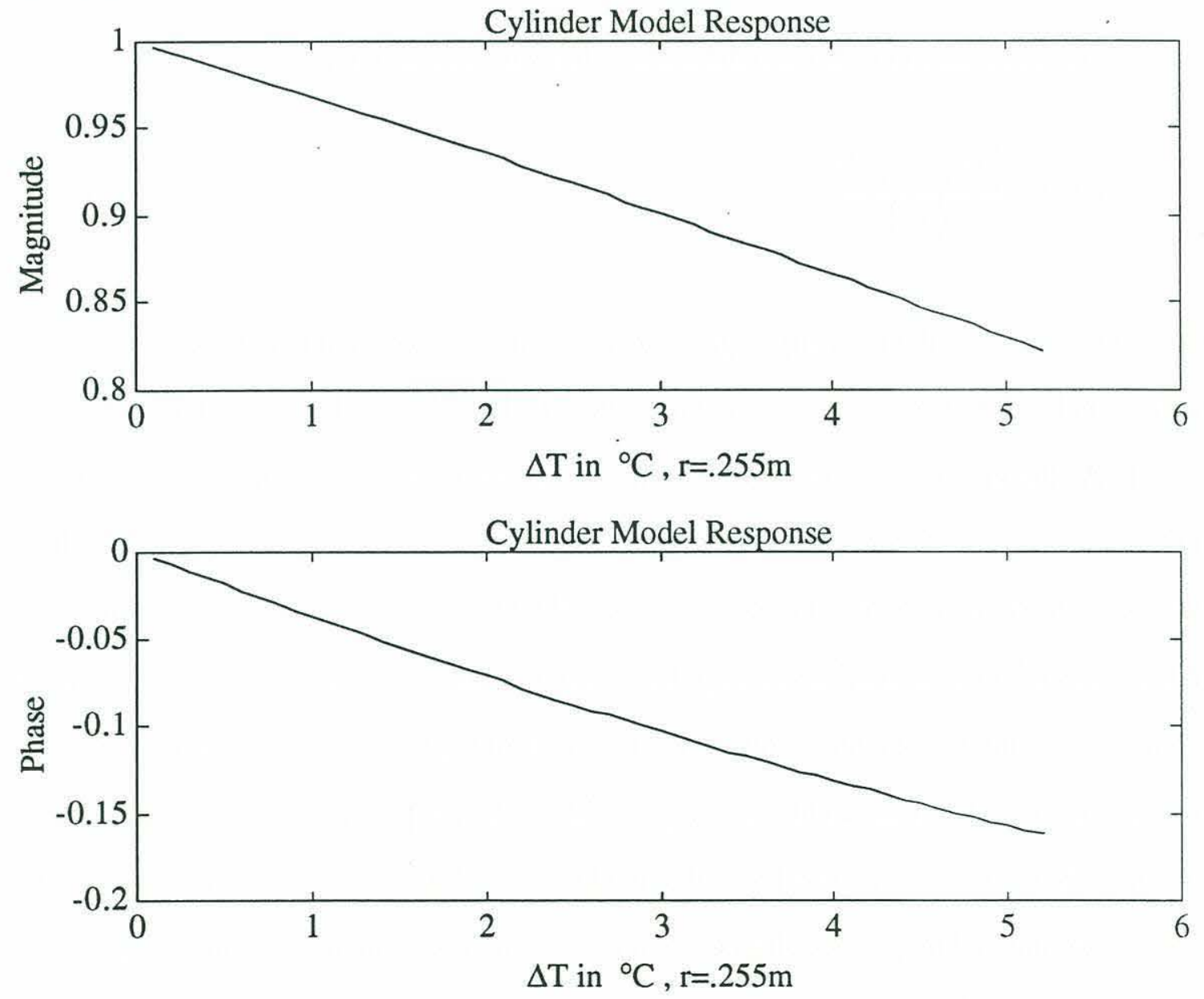

Figure 3.7 
The phase response of both the cylinder model and the Born approximation decreases with increasing $\Delta \mathrm{T}$, but the phase of the Rytov approximation increases with increasing $\Delta \mathrm{T}$.

The cylinder can be made to match any of the given magnitudes for different temperatures. For the Born approximation the ratio is approximately $8.16 * \mathrm{Cy} / /$ Born. The cylinder is used only to allow a common base for comparison of the temperature profiles in each experiment. This common base allows for analysis of the mean temperature variations within the plume.

\subsection{The Scintillation Index}

The Scintillation index is a measure of the variation of the plume.

$$
\sigma_{I}^{2}=\frac{\left\langle I^{2}\right\rangle-\langle I\rangle^{2}}{\left\langle I^{2}\right\rangle}
$$

In this circumstance the intensity is going to vary with the variation in the index of refraction. For low powers the plume varies very little and as such the $\sigma^{2}=0$ (or very small). As the variation in the index of refraction varies in magnitude more and more as the turbulence increases the $\sigma^{2}$ increases as well. At some point, if the plume becomes fully turbulent, the $\sigma^{2}$ will approach a constant that will be a measure of the maximum variation in the index of refraction achieved in plume. It may also show a decrease in $\sigma^{2}$ as power to the heater continues to increases due to the lowering of the point at which the plume becomes turbulent and the additional height with a $(-5 / 3)$ exponential temperature decrease. This may be overcome by the effects of spreading. Looking at the plume as a divergent lens the normalized magnitude should not return to unity with increasing turbulence.

In this model of a turbulent plume if the peak increase in temperature in the plume is

near $10^{\circ} \mathrm{C}$ and there is assumed to be a Gaussian distribution in time with no spreading, the 
$\sigma^{2}$ will approach $5 \times 10^{-3}$. This value is based on the Rytov magnitude and that the measured peak temperature is approximately $5 \mathrm{X}$ the mean difference from the zero power value of temperature.

The scintillation index provides a little more insight into the turbulent plume. If there is no variation in the plume the scintillation index will be zero. However, as the variance increases the scintillation index increases. In our experiment, with weak scattering, the amplitude modulation of the signal is small, so the scintillation index can be seen as a measure of the mean modulation index of the signal. This analogy allows the visualization of the signal as a carrier signal with a modulated signal produced by the variations in the index of refraction and added on to the carrier. 


\section{Chapter 4}

\section{Experimental Results}

The results of the previous section were used to look at the forward scattering through the thermal plume generated in the experiments also previously described. The results from the freshwater experiment are compared to the expected results, and then they are extended to a more complicated experiment. Each of the different heights were conducted at different times and under slightly different tank conditions. The $15 \mathrm{~cm}$ experiment was conducted at the same height as the plume temperature measurements. Because of this, the $15 \mathrm{~cm}$ data will be used for specific comparisons to the theoretical models. Extensions to the other heights will concern the nature of the plume.

\subsection{Fresh Water Tank}

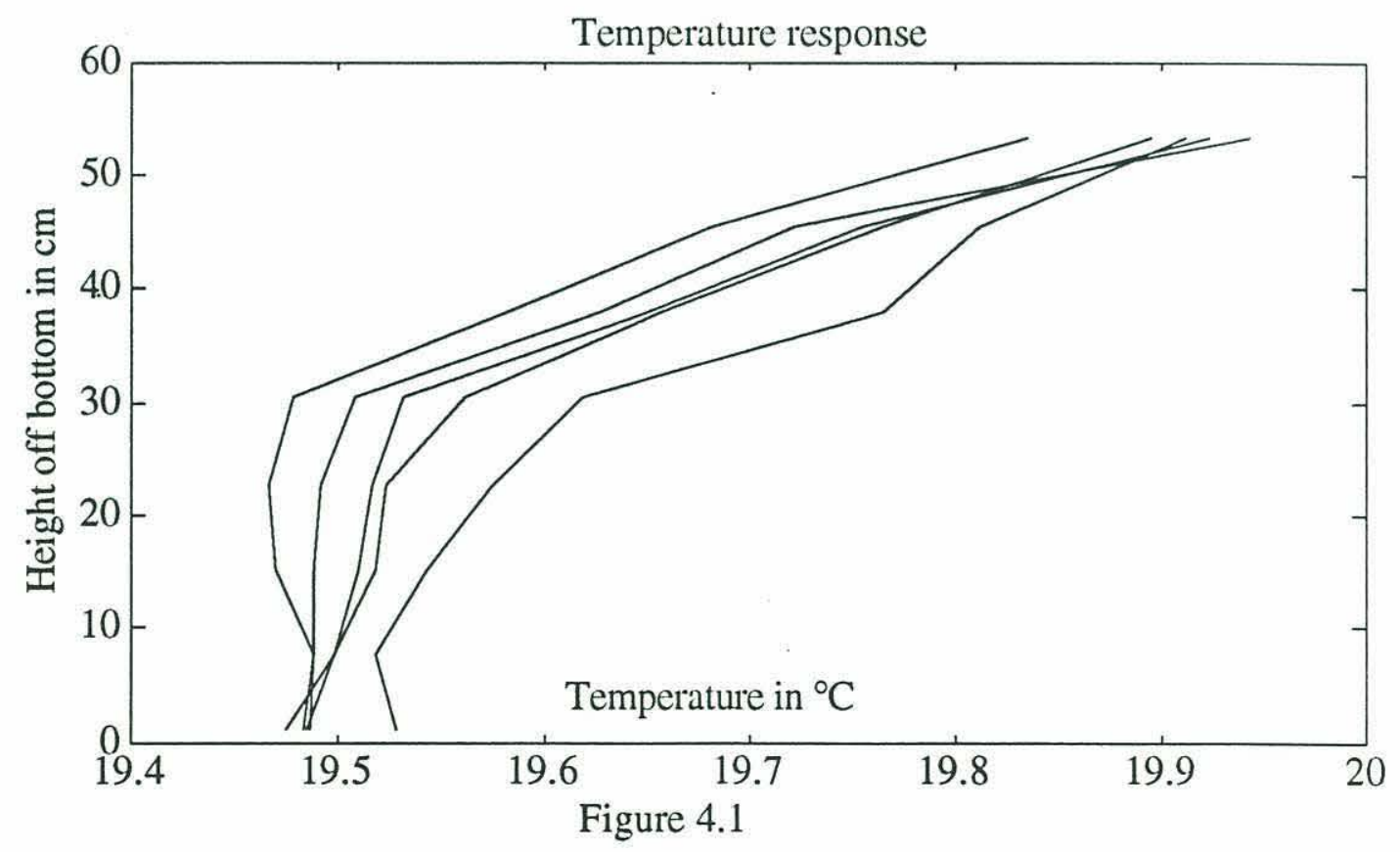




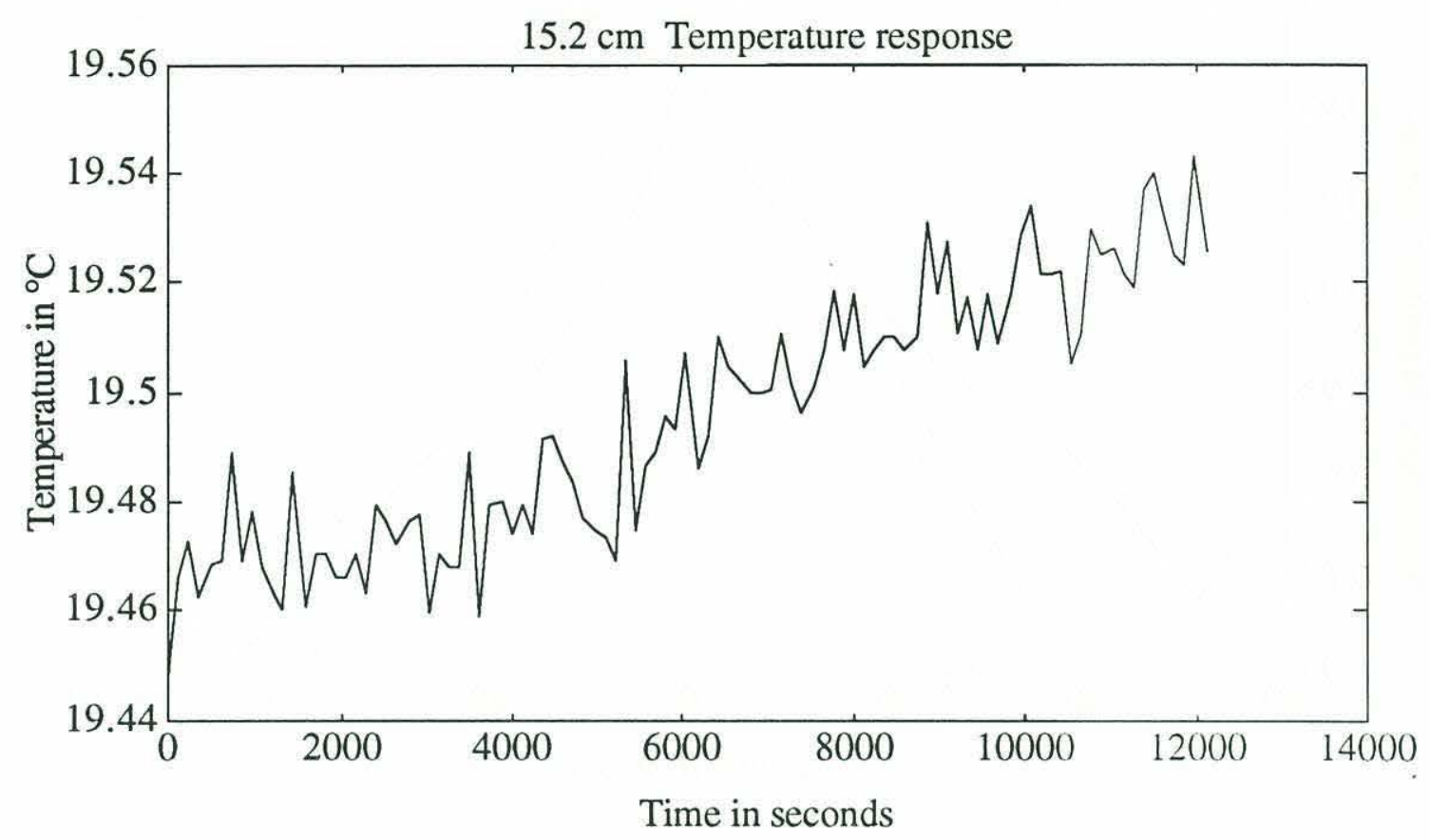

Figure 4.2

The temperature profile for the tank is shown in figure 4.1 for the duration of the experiment. Using a 10 second average the temperature difference over the duration of the experiment is $0.1071^{\circ} \mathrm{C}$. A plot of the temperature change of the thermocouple at $15.2 \mathrm{~cm}$ is shown in figure 4.2 .

\subsubsection{Magnitude and Phase Mean Response}

The temperature data at 60 volts (11.5 Watts) in Oeschger was taken at $\approx 15 \mathrm{~cm}$. The variation in temperature with power was found to be linear with power ${ }^{40}$. Figure 4.3 is a plot of the magnitude response of the Rytov method, The Born approximation, and the results for the signal at $15 \mathrm{~cm}$. The negative signs indicate decreasing power series.

\footnotetext{
${ }^{40}$ Personnel conversation with Oeschger, 24 June 1993.
} 


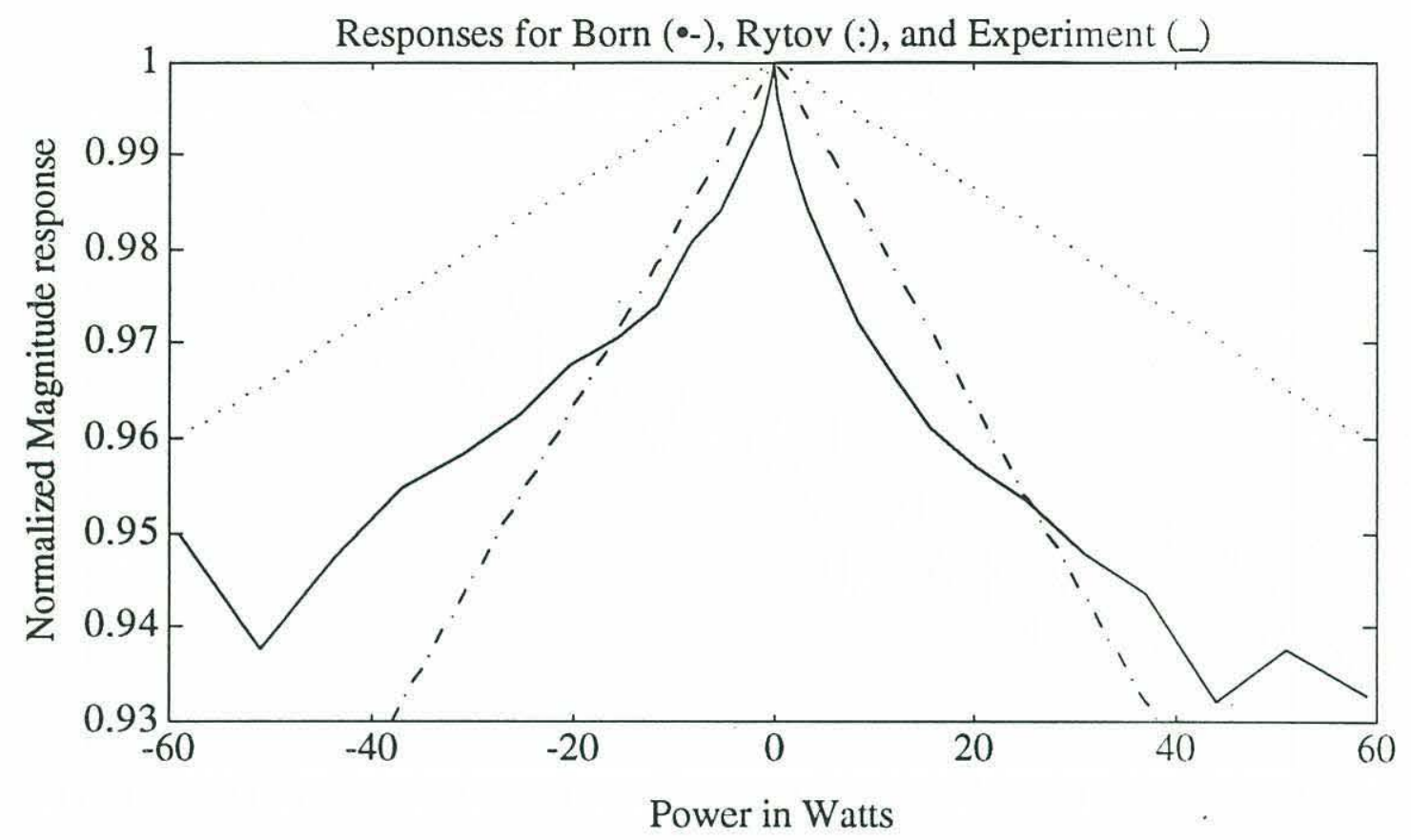

Figure 4.3

The amplitude response seems to indicate a lag time in the plume. The plumes were allowed to stabilize 5 minutes prior to each signal set and were visually checked for consistency when the plume became visible. The magnitude response of the plume parallels the Rytov approximation initially but more closely approaches the Born as the power is decreased. The response follows a similar pattern as the power is increased. A non-linear temperature vs. power response at low powers could indicate a higher temperature than the linear power vs. temperature model provides. The higher powers are also subject to increasing turbulence which changes some of the heater energy into turbulent motion and accounts for some of the flattening of the amplitude.

An effort to correct for the phase changes due to temperature variations, needs to remove the phase due to increased travel time from the $0.1071^{\circ} \mathrm{C}$ increase in ambient temperature. The slope of the phase change with power of the heater is equal to .0014. This was obtained using the mean slope for the decreasing power series and the mean slope for the increasing power series and removing the average increase to obtain the slope due to 
the changing power level alone.

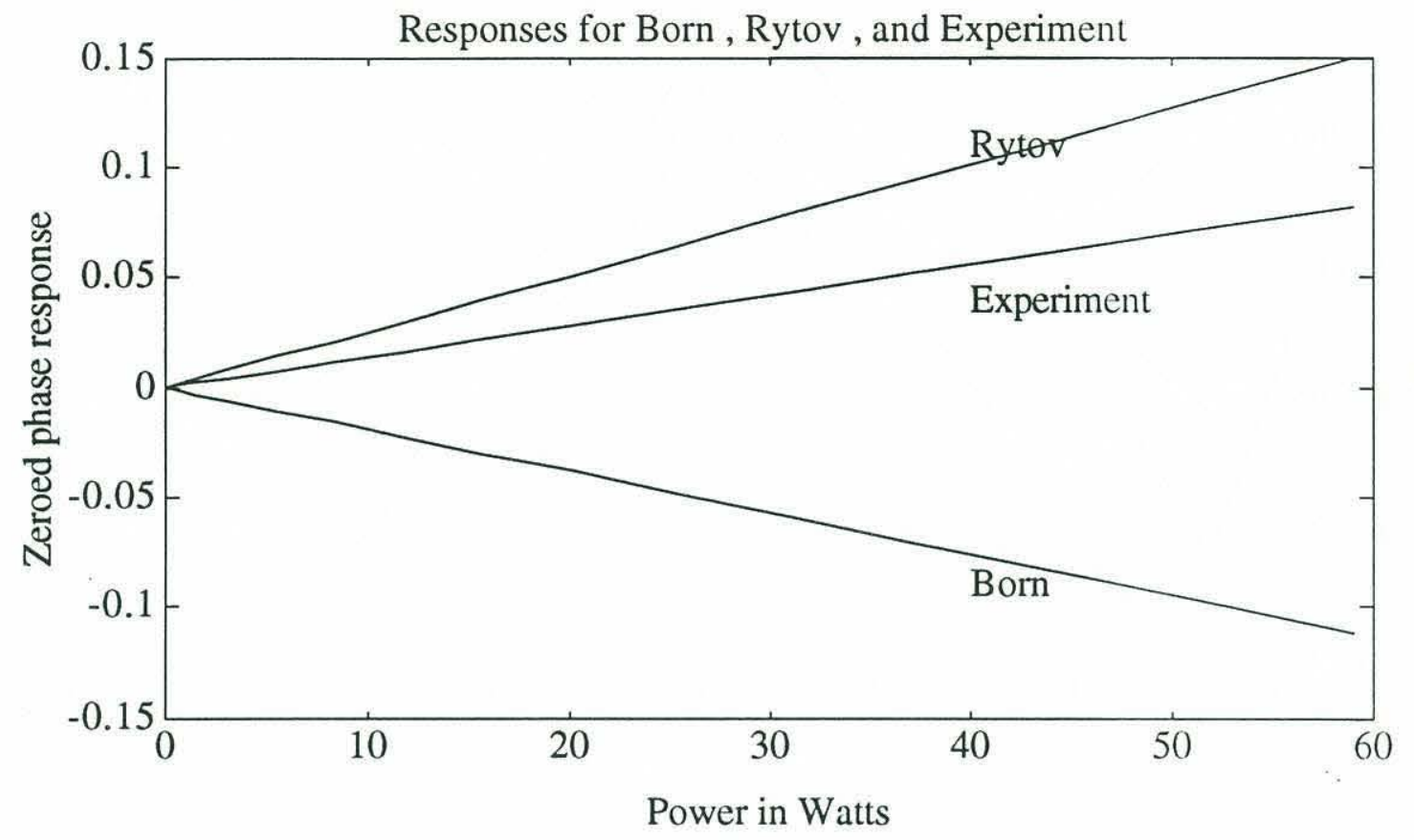

Figure 4.4

Although it appears that the Born approximation provides a better match of the amplitude response of the signal than the Rytov approximation, the phase response is clearly better matched by the Rytov approximation.

Figure 4.5 shows the magnitude responses for each of the heights used. If the magnitude responses for the different heights are compared, the effects of the decay in temperature with the height of the plume can be seen as in figure 4.5. Figure 4.6 shows this variation in terms of temperature of the cylinder.

According to discussion in chapter 3 the peak temperature should decrease linearly with height for a laminar plume. Even though the heights used were done at different times, the laminar plume equivalent temperature model plotted as a function of height for the lower powers (before the $30 \mathrm{~cm}$ signal becomes turbulent), as in figure 4.7, shows temperature decay as nearly linear with a mean slope of $-.0236^{\circ} \mathrm{C} / \mathrm{cm}$. . 


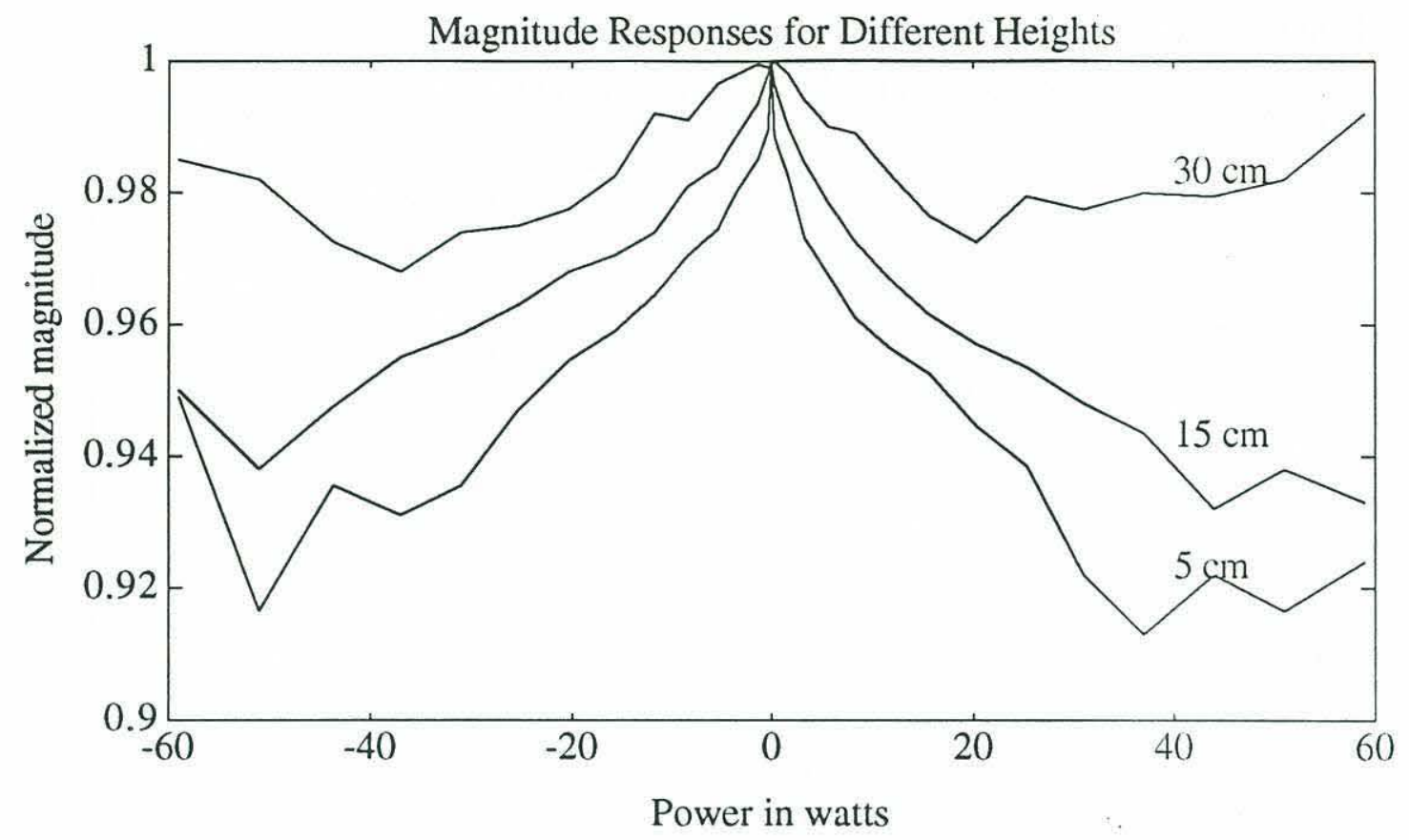

Figure 4.5

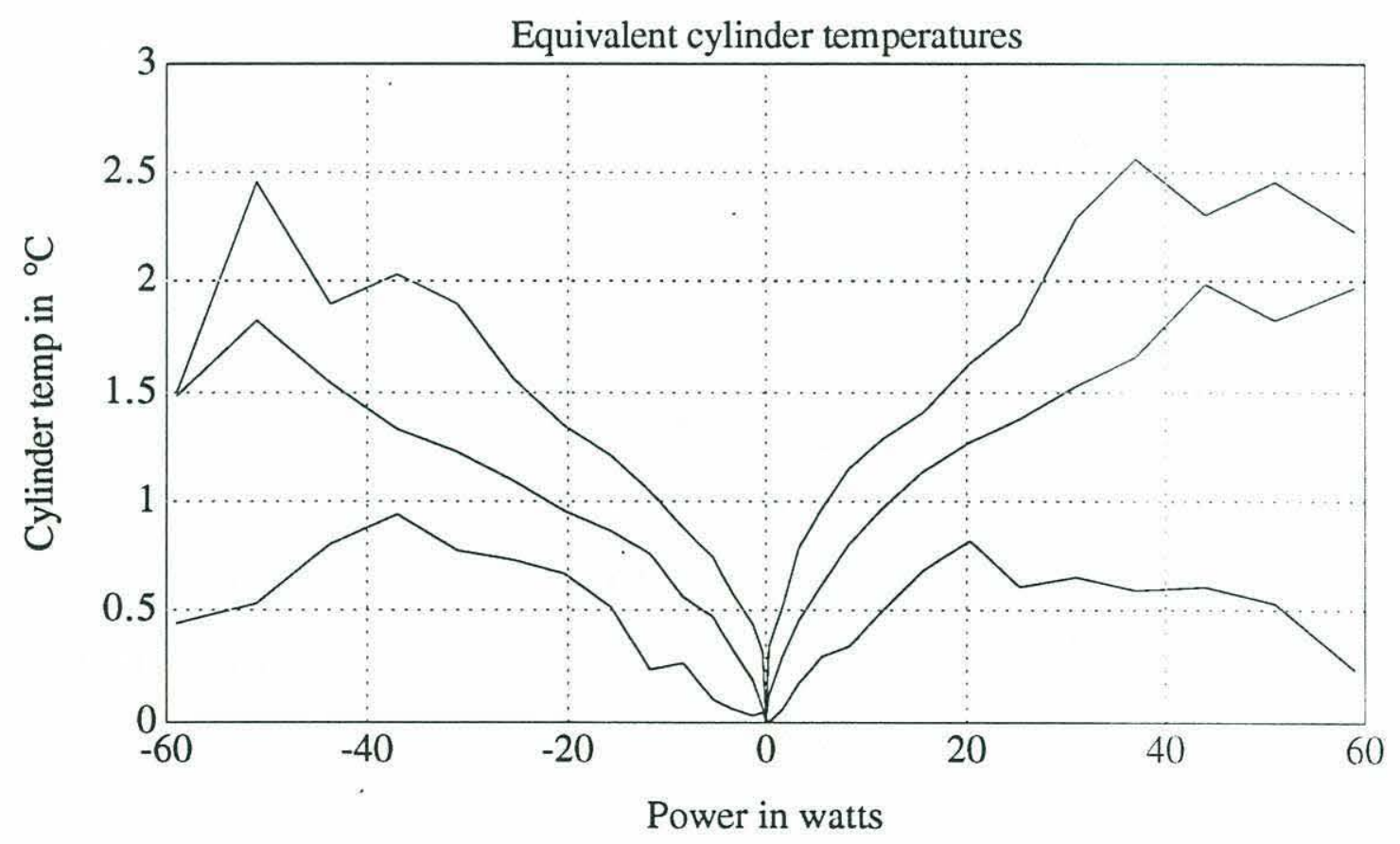

Figure 4.6 


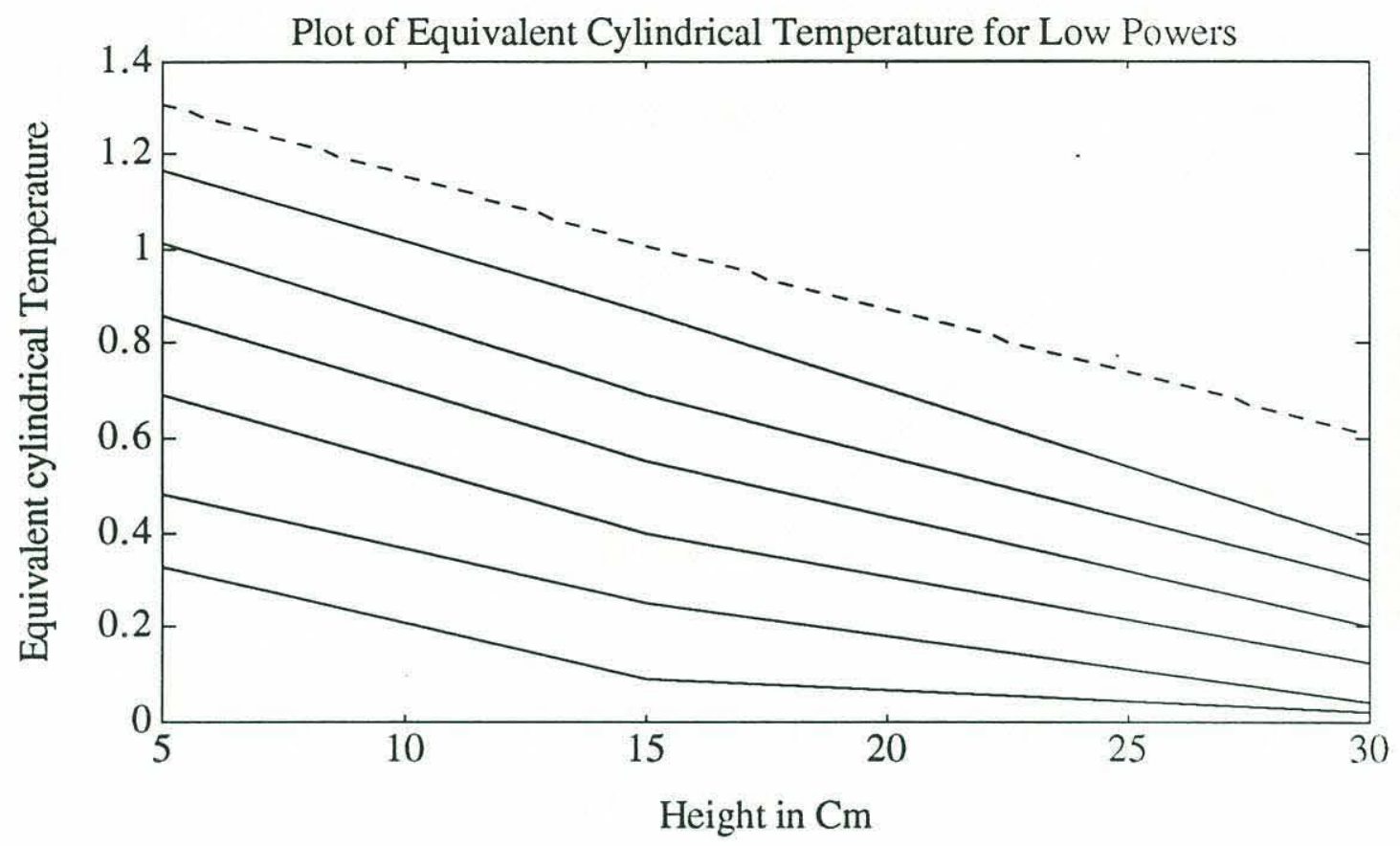

Figure 4.7

For the turbulent case, which is most nearly achieved only for the highest power level based on video and the scintillation index, a similar comparison can be determined. The radius is supposed to increase linearly with height, as such, the variance should spread linearly. The temperature decay for a turbulent system is nearly $-5 / 3$ in height, a net $-2 / 3$ magnitude vs. height exponential decay in signal response is expected. However, the limited number of available turbulent signal responses ensures a skewed result. To match the signal at $5 \mathrm{~cm}$ a factor of 0.2535 is needed. Figure 4.8 shows the plot of the highest power responses for each of the heights. The signal at $15 \mathrm{~cm}$ appears not to have been fully turbulent at the power level shown. The error at $30 \mathrm{~cm}$ will be significant for the constant used at $5 \mathrm{~cm}$. Clearly there is not enough data to do a good comparison. 


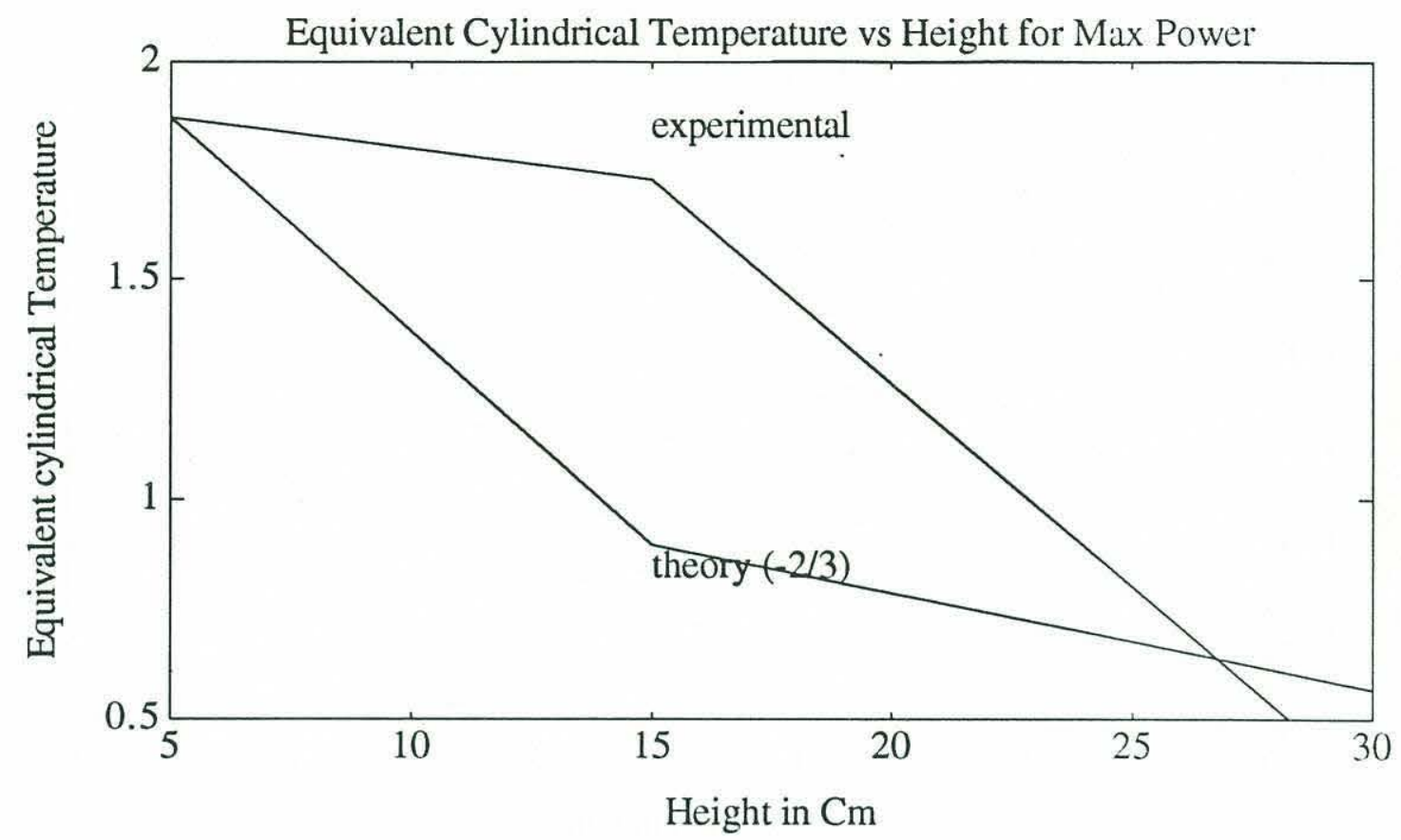

Figure 4.8

\subsubsection{Frequency Response}

The propagated signal time series with the signal sampled at $100 \mathrm{~Hz}$ when the plume is transitioning to turbulent appears in figure 4.9. A Fourier transform of the signal in figure 4.9 illustrates that most of the signal is well below $2 \mathrm{~Hz}$ and is shown in figure 4.10. The sampling rate was subsequently reduced to $10 \mathrm{~Hz}$ which allowed a closer look at the low frequency modulation of the signal. This sample rate (actually the pulse repetition rate of the signal used) lowered the highest discernible frequency to $5 \mathrm{~Hz}$ and reduced the frequency bin size from $0.098 \mathrm{~Hz}$ per bin to $0.0098 \mathrm{~Hz}$ per bin.

If the FFT of the signal magnitude squared at $30 \mathrm{~cm}$ is examined, and a rough $\chi 2 \mathrm{fit}$ is made, a close approximation to the $\chi^{2}$ distribution can be seen. Figure 4.11 uses a $\chi^{2}$ distribution of order two which indicates an underlying Gaussian distribution of the real 
and imaginary parts of the received amplitude signal.

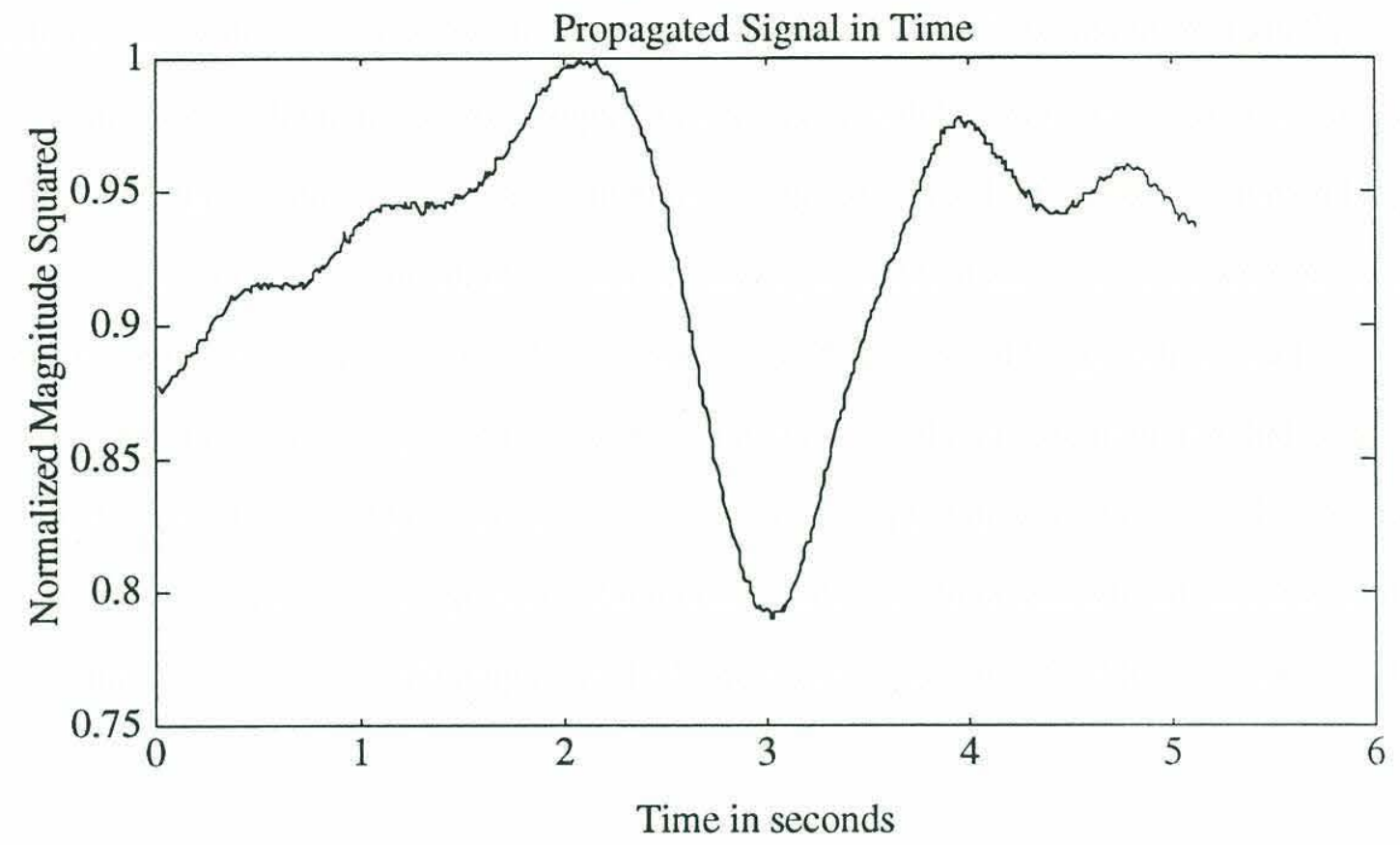

Figure 4.9

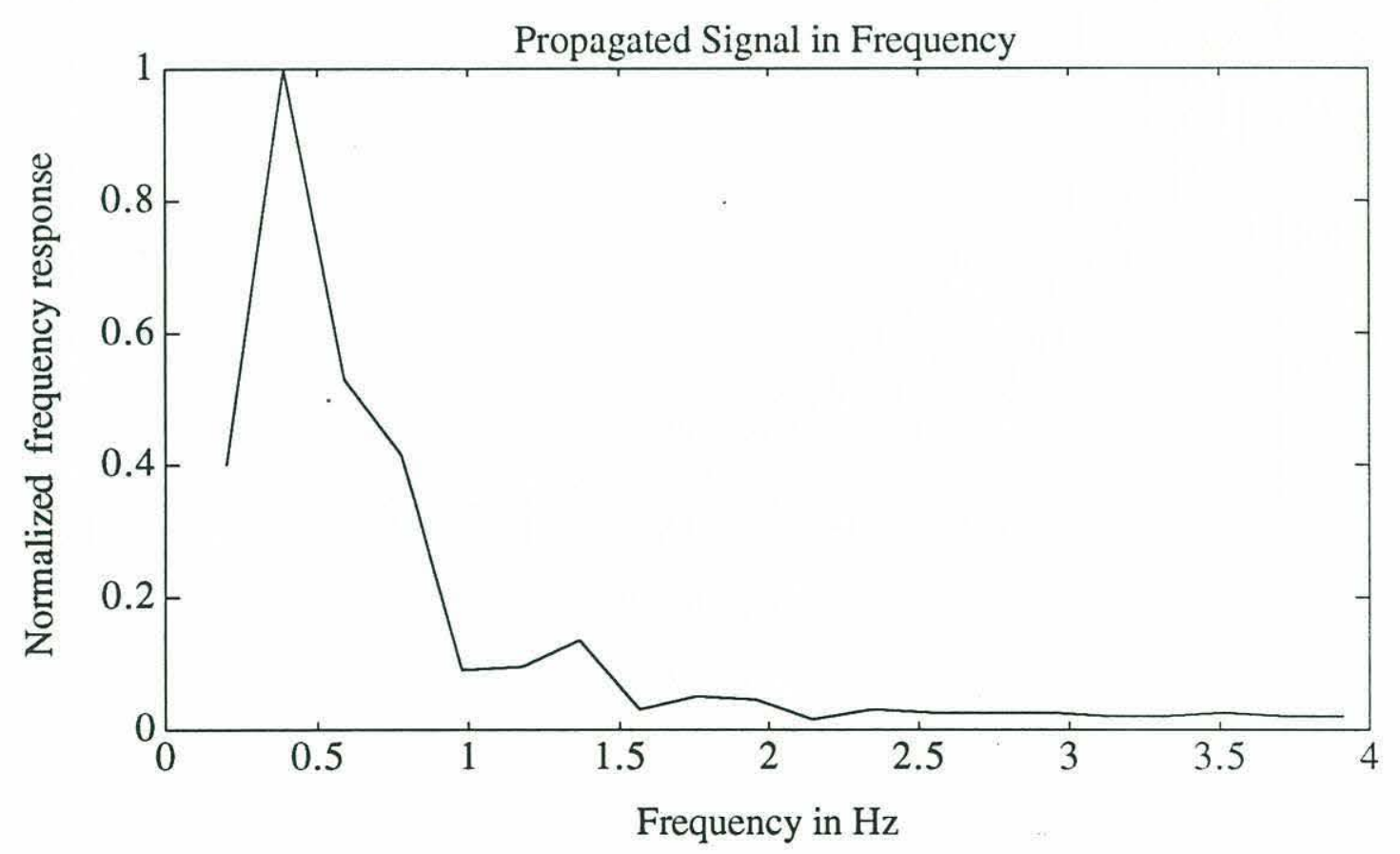

Figure 4.10 
The $\chi 2$ distribution arises from the sum of the squares of the two Gaussian variables and is of the form of an exponential decay. The lower frequencies dominate this spectrum of signal response. Using $0.5 \mathrm{~Hz}$ as a rough estimate of the size of the inner scale at 0.707 $\mathrm{Hz}$ is $3.8 \mathrm{~cm}$. This indicates that the larger eddies dominate the modulation of the signal. If the of the signal for several heater powers is shown, the increasing modulation as the turbulence increases can be seen as in figure 4.12 . The signal at $15 \mathrm{~cm}$ never fully became turbulent as shown in the plot of the temperature vs. height plot in figure 4.8 and as will be seen in the discussion of the scintillation index. The figure does demonstrate the initial appearance of higher frequencies before the low frequencies become dominant.

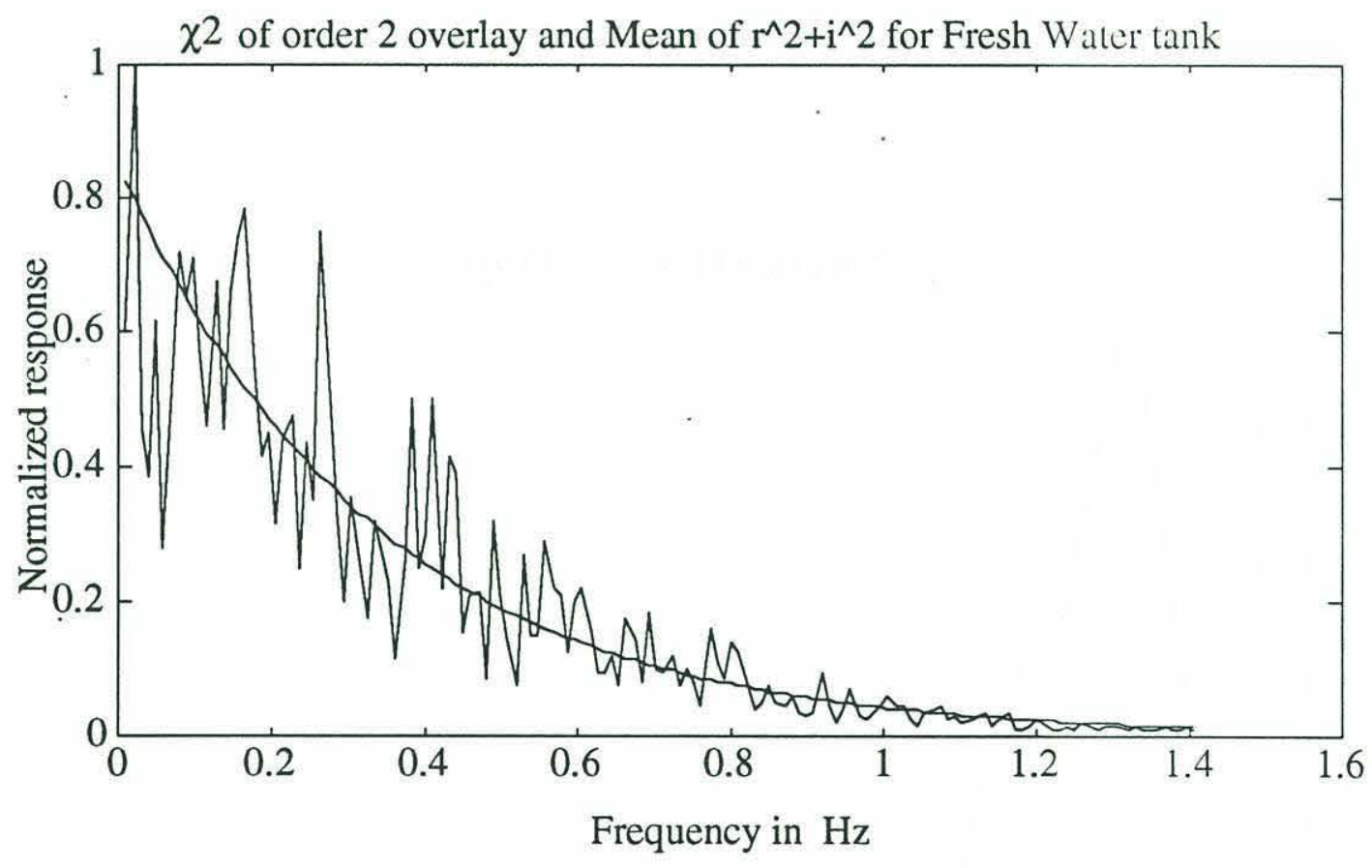

Figure 4.11 


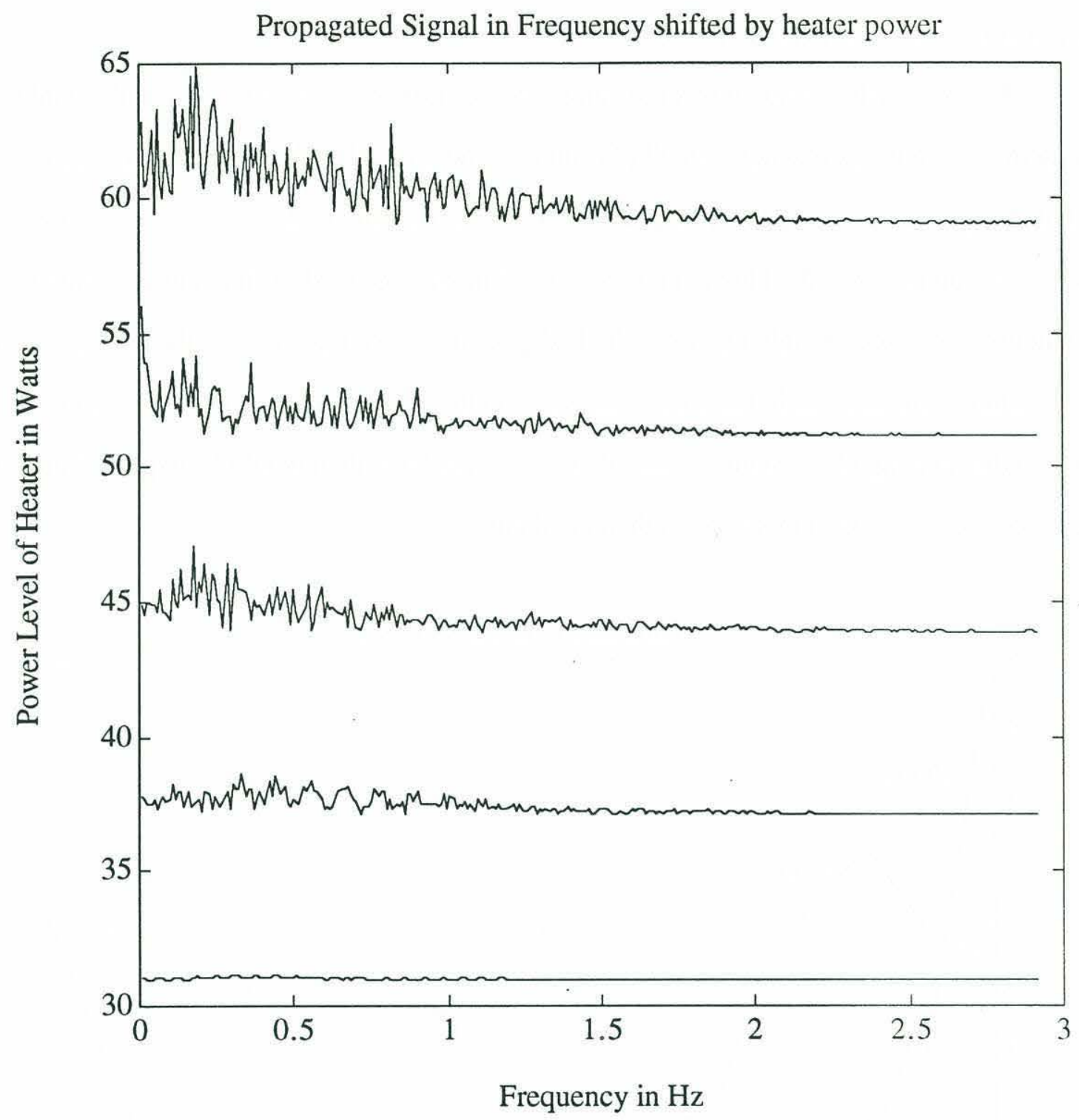

Figure 4.12

\subsubsection{Scintillation Index}

The scintillation index was determined for each power level for each of the heights. Figure 4.13 illustrates the significant points discussed in chapter 3 . As the plume becomes turbulent, departing from the laminar plume, the scintillation index starts to increase. The scintillation index increases until it reaches a plateau, where the turbulence is fully 
developed. In addition the plume may not be turbulent at all heights above the source but may become turbulent at some point above it.

The $5 \mathrm{~cm}$ scintillation index indicates that the fluid in the tank was not fully stable. The scintillation index reaches a small plateau for power equal to 0 watts indicating some turbulence present either in the motion of the water in the tank or due to some disruption of a stratification of the tank. However, this disruption was not as significant as the plume turbulence generated at higher powers. This slight turbulence does lead to the similar point of departure from laminar in the $5 \mathrm{~cm}$ plume and in the $15 \mathrm{~cm}$ plume. This disruption also leads to the leveling of the scintillation index at a lower level than would be expected due to the higher peak temperatures at $5 \mathrm{~cm}$ than at $30 \mathrm{~cm}$.

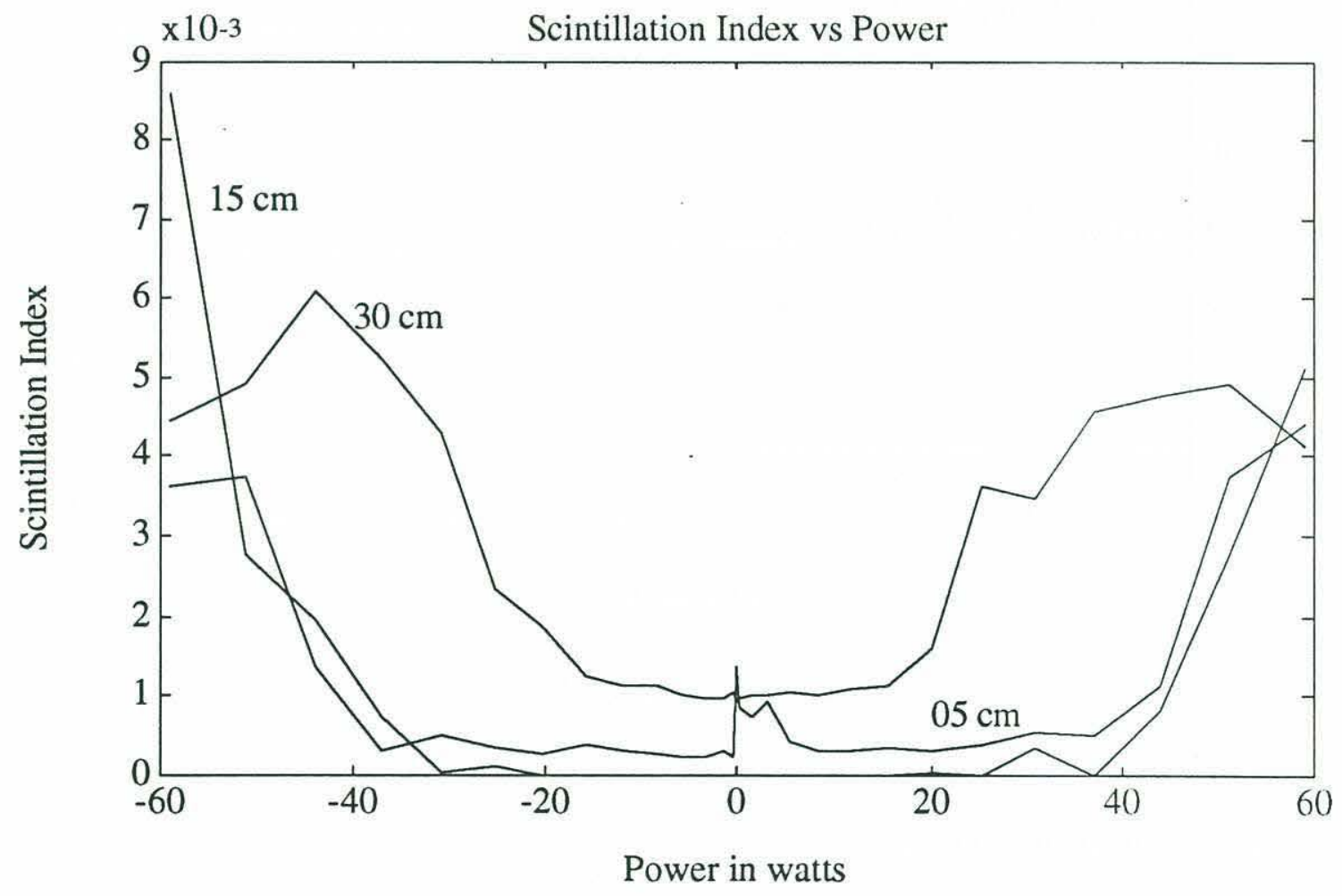

Figure 4.13

The $15 \mathrm{~cm}$ scintillation index never reaches a plateau and thus the $15 \mathrm{~cm}$ plume never reaches full turbulence. The scintillation index does indicate that some sort of disturbance has been developed as the experiment has proceeded as shown by the lower 
slope for the increasing power portion of the experiment. The $15 \mathrm{~cm}$ scintillation index also indicates that the higher peak temperature within the plume than at $30 \mathrm{~cm}$ leads to a higher scintillation index. The peak scintillation index of $8 \times 10^{-3}$ corresponds to a peak of $25^{\circ} \mathrm{C}$. This is a fairly high value and is based on the Rytov approximation and the $5 \mathrm{x}$ the equivalent temperature for the mean difference from the no power case to obtain the peak temperature. The Born approximation would lead to a peak temperature of $10^{\circ} \mathrm{C}$. That creates a reasonable range for estimation of the peak temperature from the scintillation index. If the variance of the plume thermal profile increases by a factor of 9 ( $b$ increases by a factor of 3 , physically spreads by a factor of 3 ) the temperatures become $0.4{ }^{\circ} \mathrm{C}$ for the Born and $2.8^{\circ} \mathrm{C}$ for the Rytov.

Since the peak temperature at $15 \mathrm{~cm}$ has been measured and is at least $5.2{ }^{\circ} \mathrm{C}$ at 11.5 Watts, the factor by which $b$ has increased for this case is at most 2.2. for the Rytov approximation and 1.3 for the Born approximation. Unfortunately, precise statements cannot be made since the experiment did not include a detailed measurement of the plume temperature distribution at all power levels. The variations in the scintillation index are due in general to either the increased radius of the plume or to an increase in peak temperature of the plume.

The scintillation index for $30 \mathrm{~cm}$ not only reaches the plateau it even displays a possible decrease in the scintillation index due to a lower height for the point of departure from laminar. This decrease is briefly discussed in the previous chapter.

The freshwater tank data provides an a illustration of the plume in both its laminar and turbulent states. The general trends in the effect the changing state of the plume has on the propagated signal are discussed and illustrated. This information is of little practical value if they cannot be used in a more realistic application. 


\subsection{Comparison to Salt Water Tank Data}

\subsubsection{Magnitude and Phase}

The above discussion of the plume in the fresh water tank provides some guidance for the anticipated results for a thermal plume. The small fresh water tank provides a well controlled medium in which to create a controlled plume. In the real world outside of this tank, things are not and cannot be controlled. As such, the factors by which we have estimated the parameters of the plume may not provide the same insight as in the controlled situation.

The large tank used in this experiment is subject to stratification in salinity and in temperature. In addition the orientation of the tank and the presence of air conditioning at one end of the tank creates internal waves within the tank. These internal waves greatly affect the propagated signal over time. The tank also has some suspended particulates and gas bubbles which can greatly affect the signal.

The effects of the above phenomena can be seen in the signals and are easily seen in the transmitted pulses. The particulates create discrete drop outs in the time series magnitude of the transmitted signal. Both the particulate and the bubbles have such sharp discontinuities in the coefficient of refraction that the propagated signal is greatly diminished. The internal waves within the tank are of a longer time scale than the sample time periods used in the experiment. An example of the received magnitude for a single pulse train is shown in figure 4.14 for a 44 watt heater power and a height of $15 \mathrm{~cm}$ above the plume.

In the experiment conducted in the large tank the heater power was initially at zero watts and then increased to the peak value near 60 watts. Then the power was reduced. This is the opposite of the method used in the smaller tank. The plots are reversed to present the same orientation as the previous plots. So that for the decreasing power series the power has a negative sign in front. 


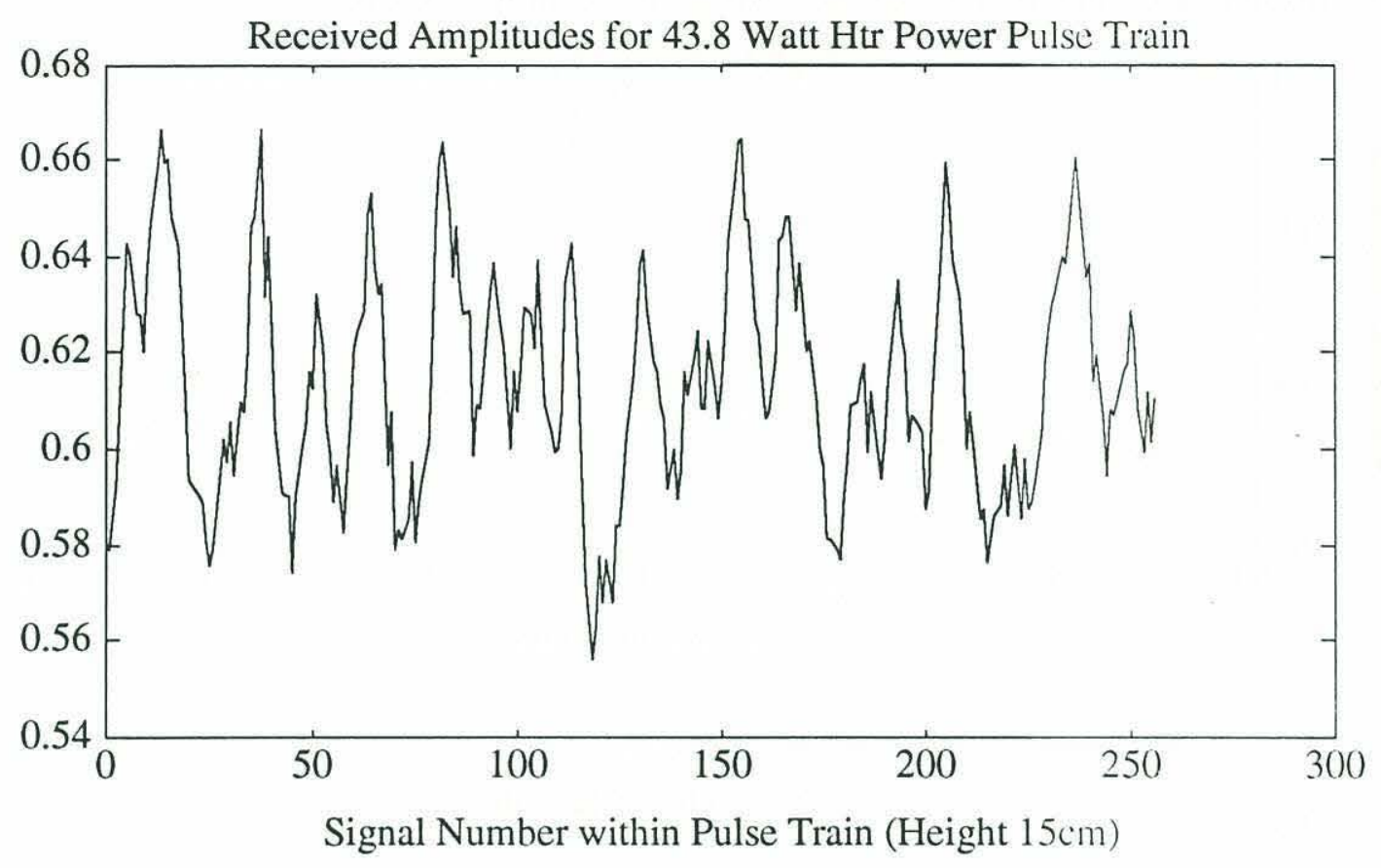

Figure 4.14

The magnitude response of the signal at $5 \mathrm{~cm}$ in height are shown below in figure 4.15 .

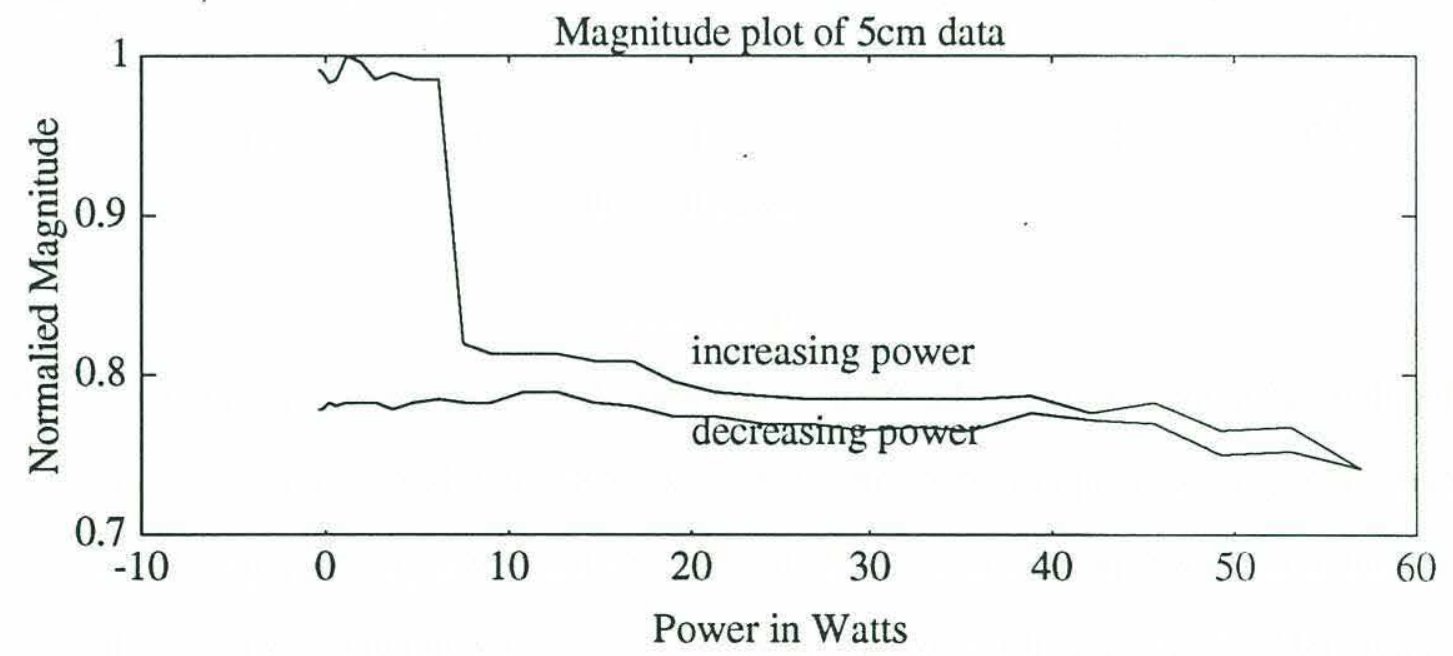

Figure 4.15

This plot is shown first to illustrate some of the problems inherent in the use of a more realistic media. The large magnitude decrease is probably due to the passing of a large eddy between the source and the receiver. Unfortunately, it didn't return. The plots 
organized in the same manner as previous plots are shown below.

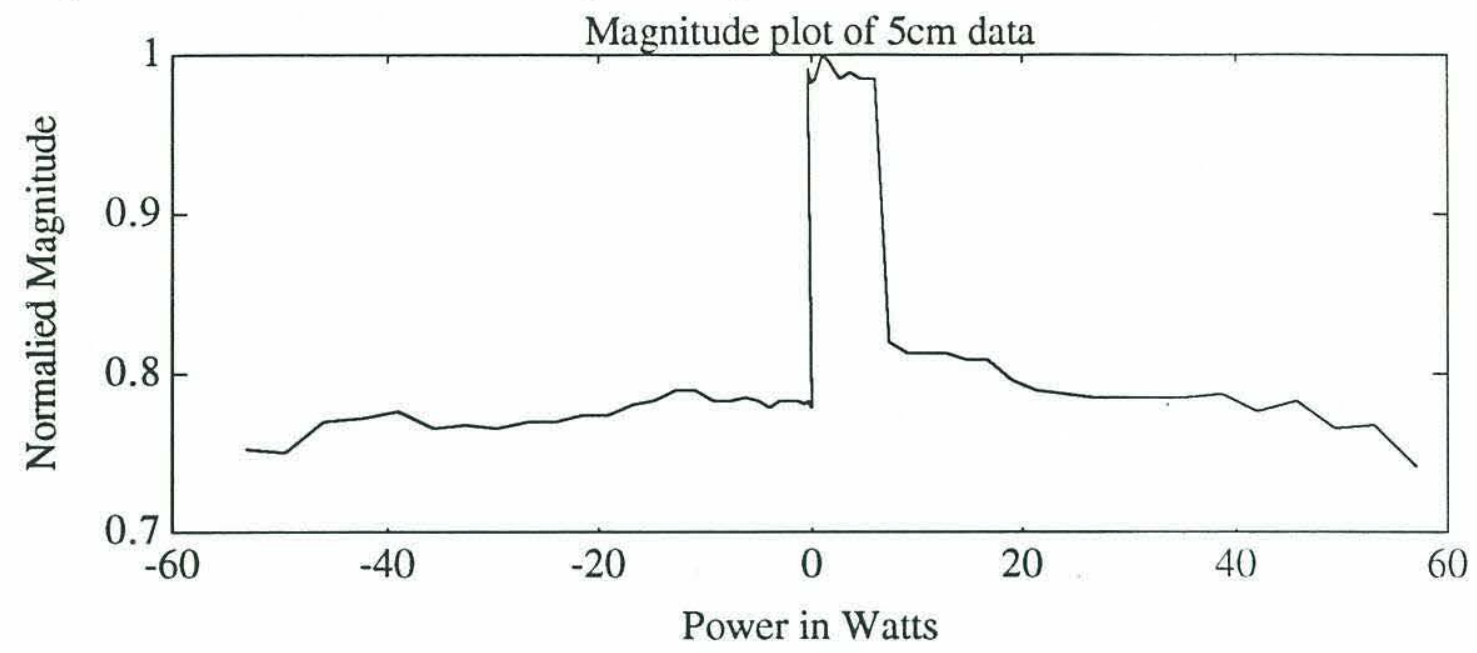

Figure 4.16

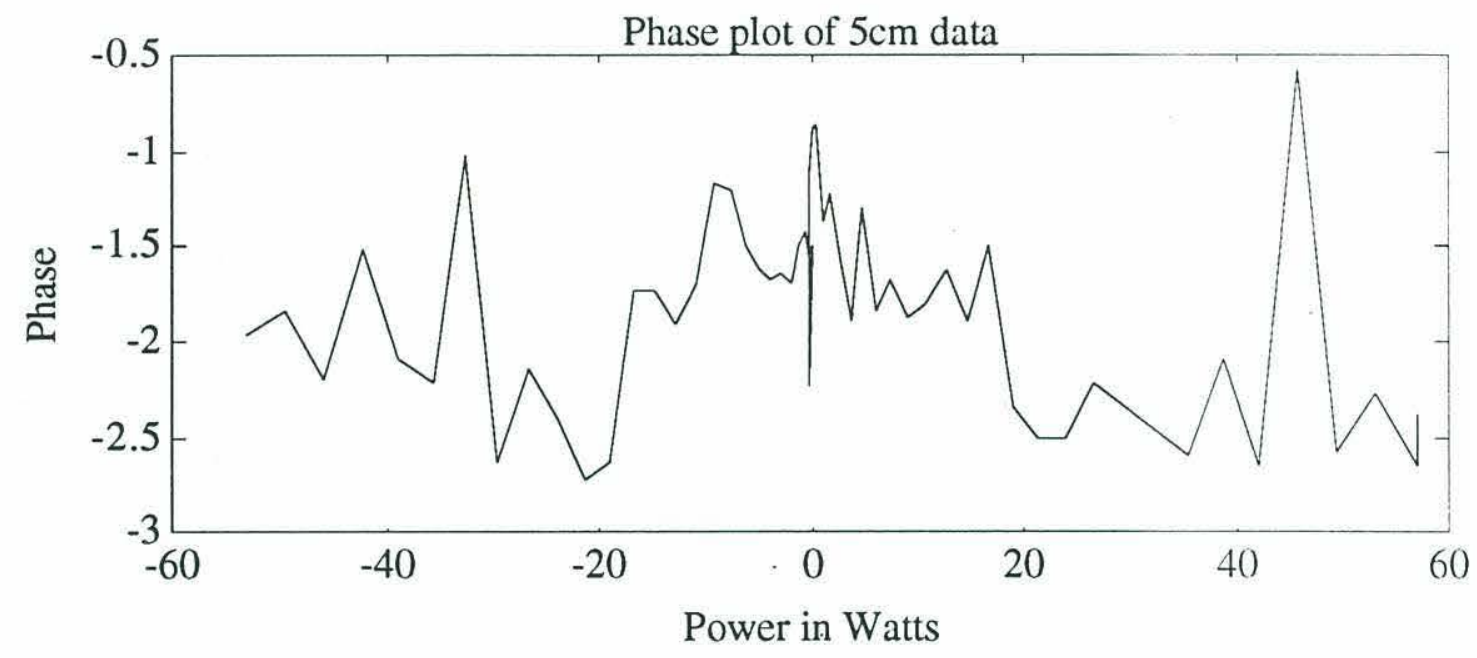

Figure 4.17

The phase plot does not show the effect of the eddy, but it does not show the effect of the heater raising water temperature either. The phase seems nearly random but closer to constant at the lower powers as it should be. The higher power phases appear to be much more variable. The scale of the variability in phase is more than ten times that of the small tank.

The magnitude plot for the $15 \mathrm{~cm}$ experiment is plotted along with the Born and Rytov approximations in figure 4.18. The data does not appear to have been subject to an eddy but merely to the smaller scale fluctuations in the media. 


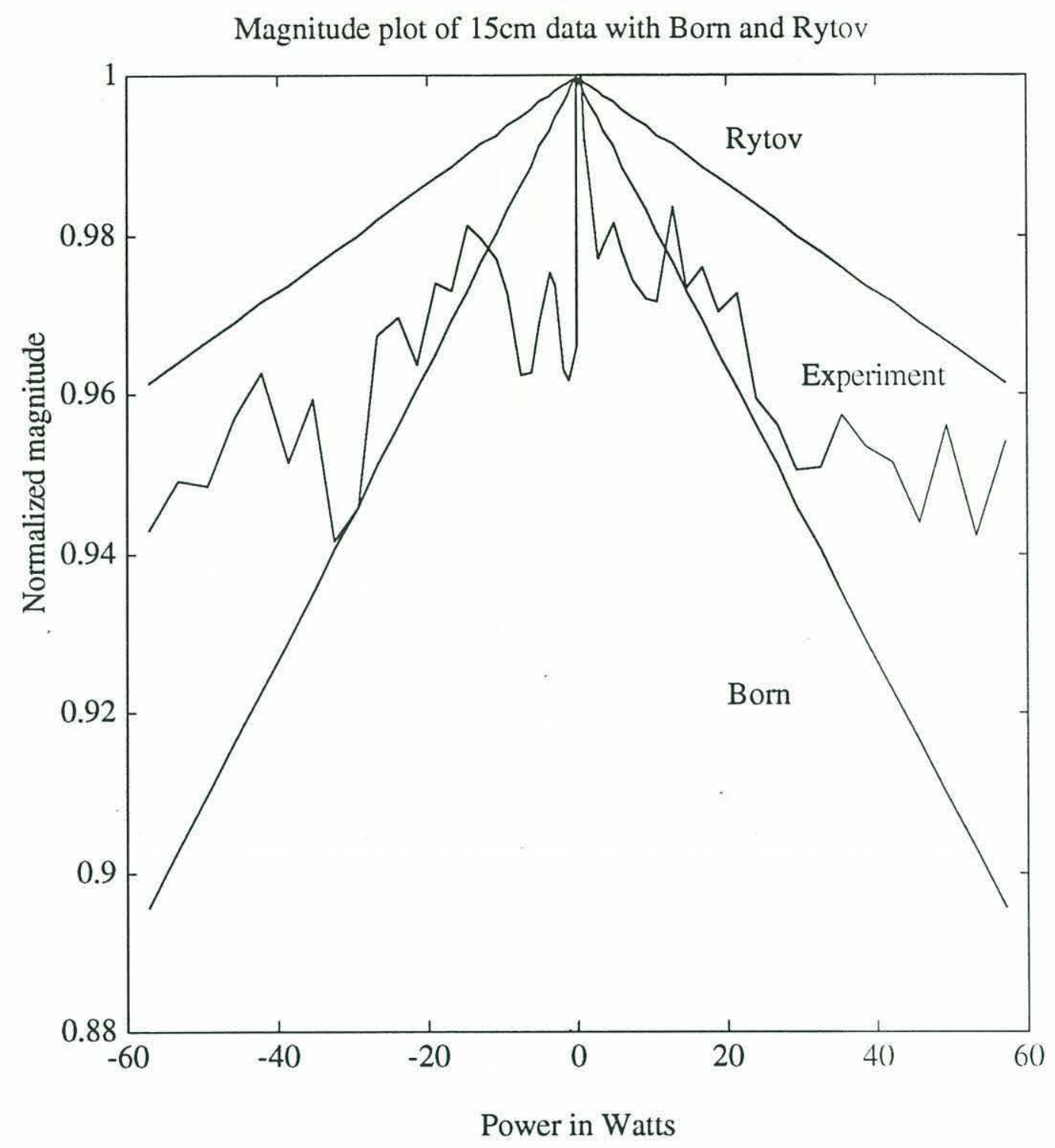

Figure 4.18

It appears that the Born and Rytov approximations are still valid when the system can have some of the larger disturbances removed. The phase however continues to be nearly random as shown in figure 4.19 . The phase varies over the entire $2 \pi$ range with very small variations in power. 


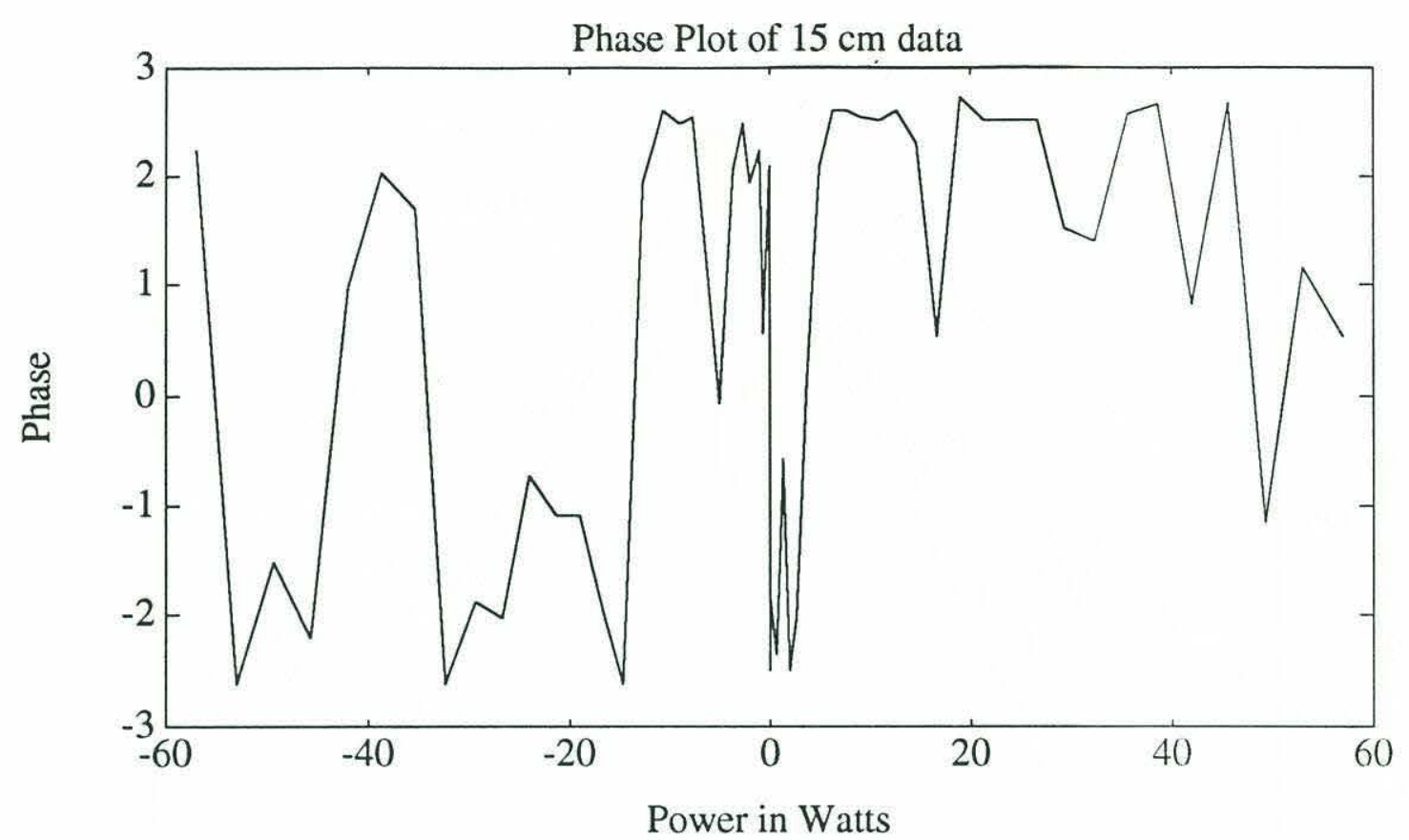

Figure 4.19

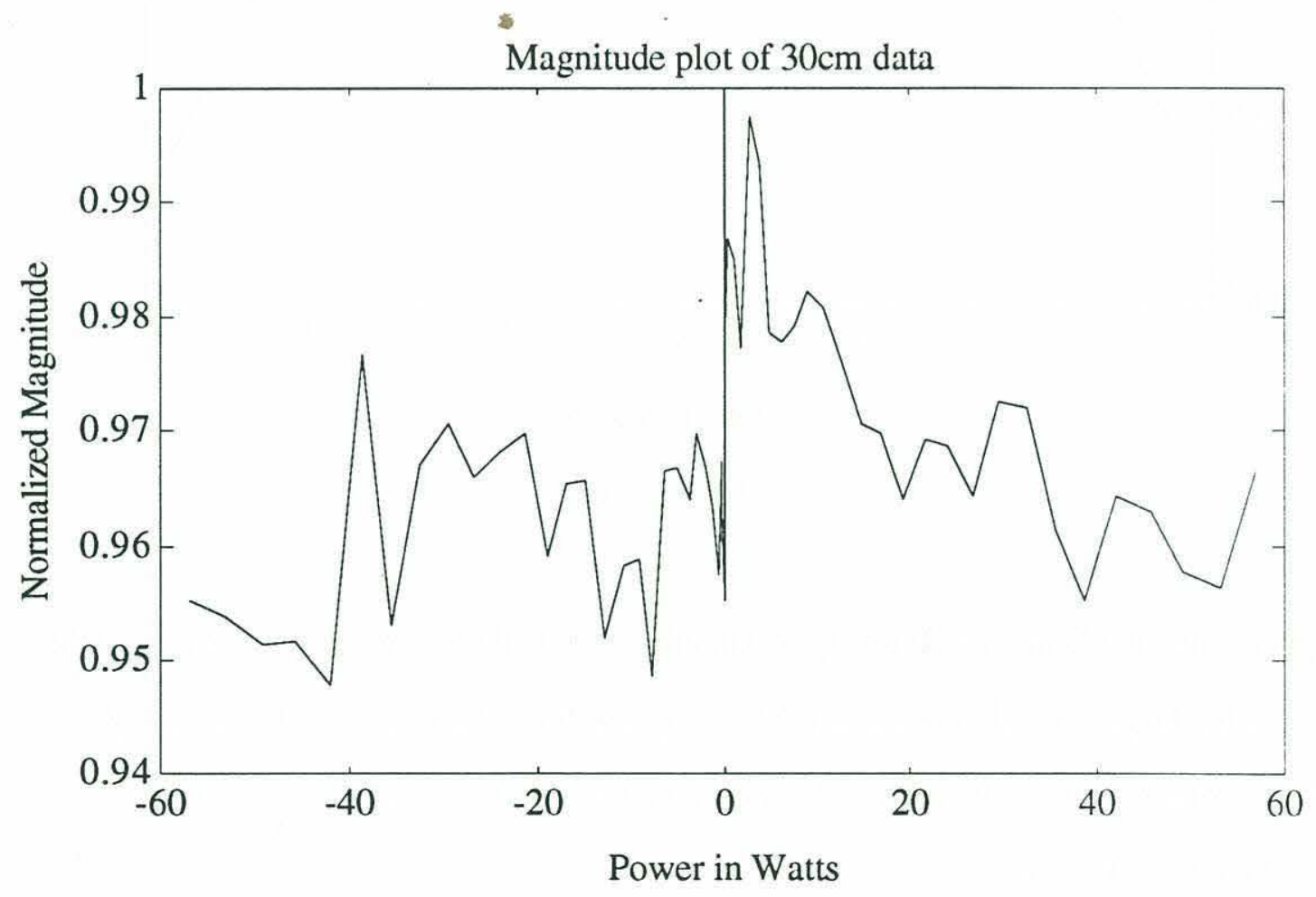

Figure 4.20 
The plot of magnitude at $30 \mathrm{~cm}$ in figure 4.20 , allows some of the decrease in amplitude with increasing power to be seen, but also shows the effect of becoming turbulent at a lower power than the $15 \mathrm{~cm}$ case.

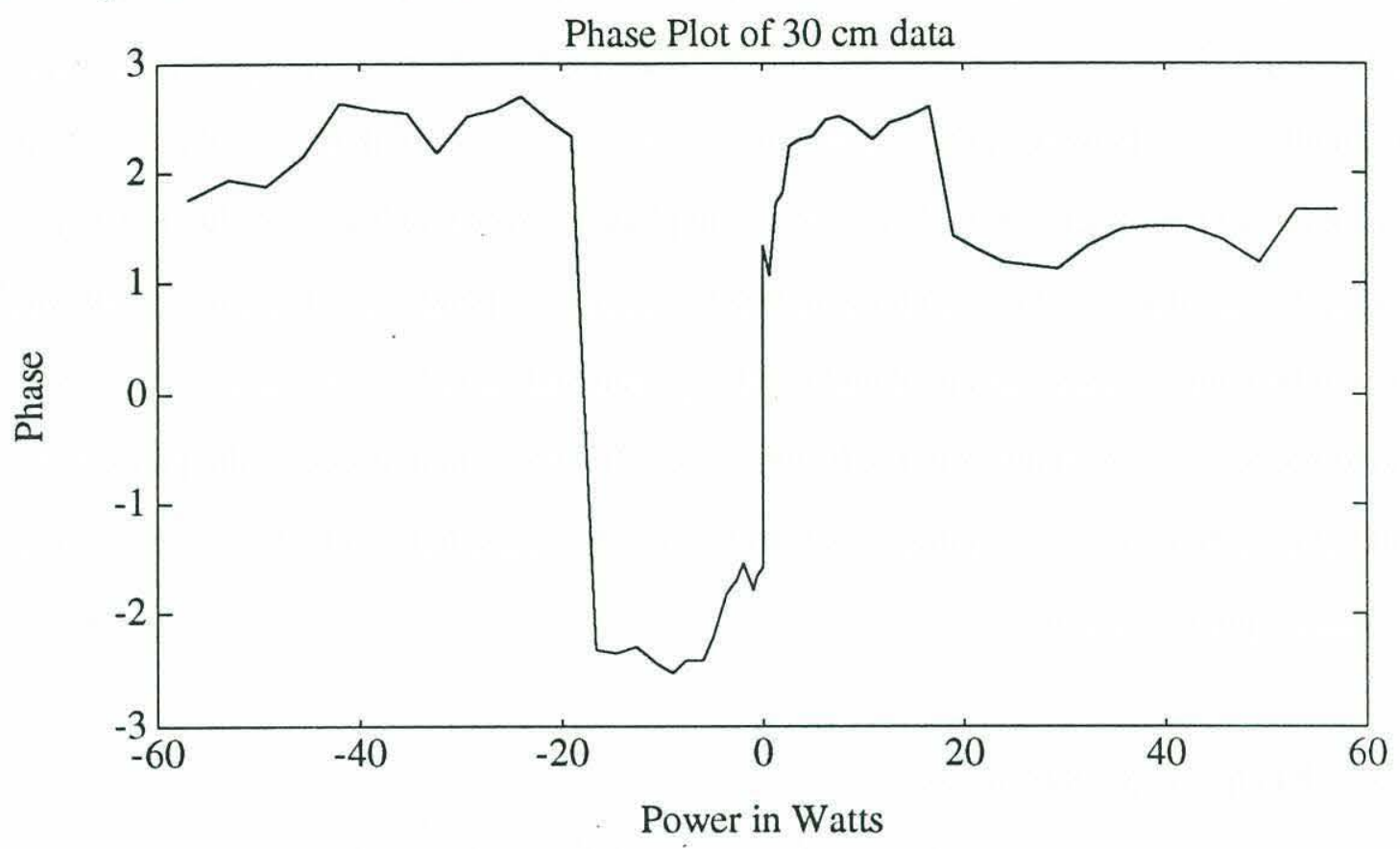

Figure 4.21

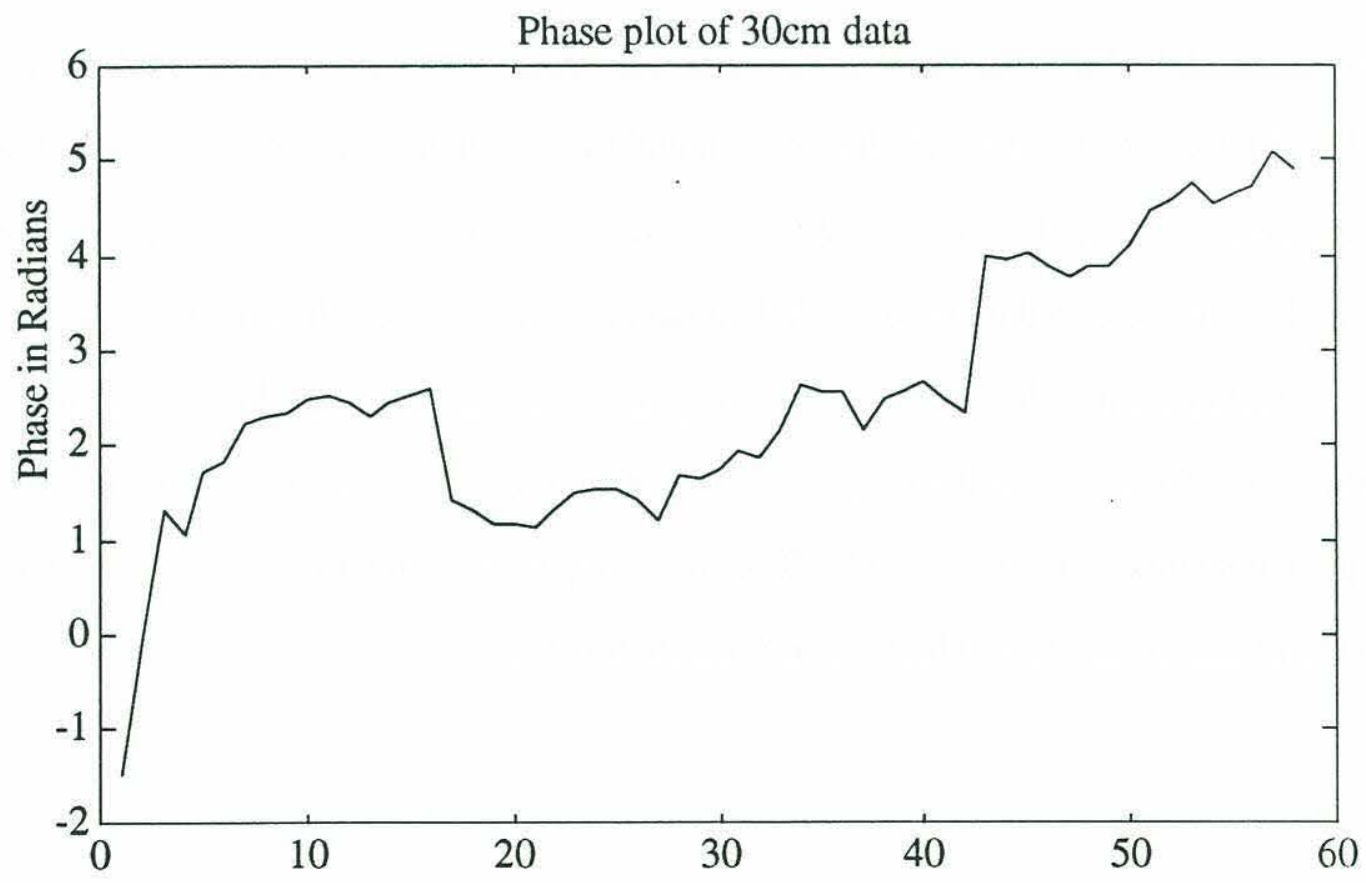

Run number (power $1=0$ watts, power $30=57$ watts, power $58=0$ watts) Figure 4.22 
The phase continues to be difficult to resolve. The phase response at $30 \mathrm{~cm}$ is shown in figure 4.21 . The unwrapped phase is shown in figure 4.22 for the $30 \mathrm{~cm}$ case and demonstrates the range over which the phase changed throughout the experiment. This plot seems to illustrate the similar increase in phase as the experiment progressed as seen in the smaller tank. However, the increase in phase for the small tank was only 0.8 radians during the $15 \mathrm{~cm}$ experiment. This increase in phase is over 4 radians and due to daily heating in the room. In order to remove this trend, the temperature of the entire tank would have to be continuously measured and carefully removed from the trend and variations in sound speed due to variations in the heating rate. The shear magnitude of the phase change and the accuracy of the measurements over this range would have to be fine enough to see a 0.1 radian phase variation.

\subsubsection{Frequency Response}

Another measure of turbulence is the modulation of the magnitude time series. In the small tank a $\chi 2$ distribution was used. The $\chi^{2}$ distribution is shown with the normalized FFT of the magnitude squared of the signal from the salt water tank in figure 4.23. Figure 4.23 shows a $\chi 2$ distribution and the dominance of the lower frequencies within the spectrum. The fit is not nearly as good as for the fresh water tank, but this can mostly be attributed to the limited turbulent data at $15 \mathrm{~cm}$ in the salt water tank.

A Fourier transform of the phase response helps illustrate the lack of obvious phase

modulation. As shown in the magnitude and phase plots the phase does not show as simple a response as the magnitude. The frequency plots of the magnitude, figure 4.23 , and the phase, figure 4.24 , clearly demonstrate this fact. 


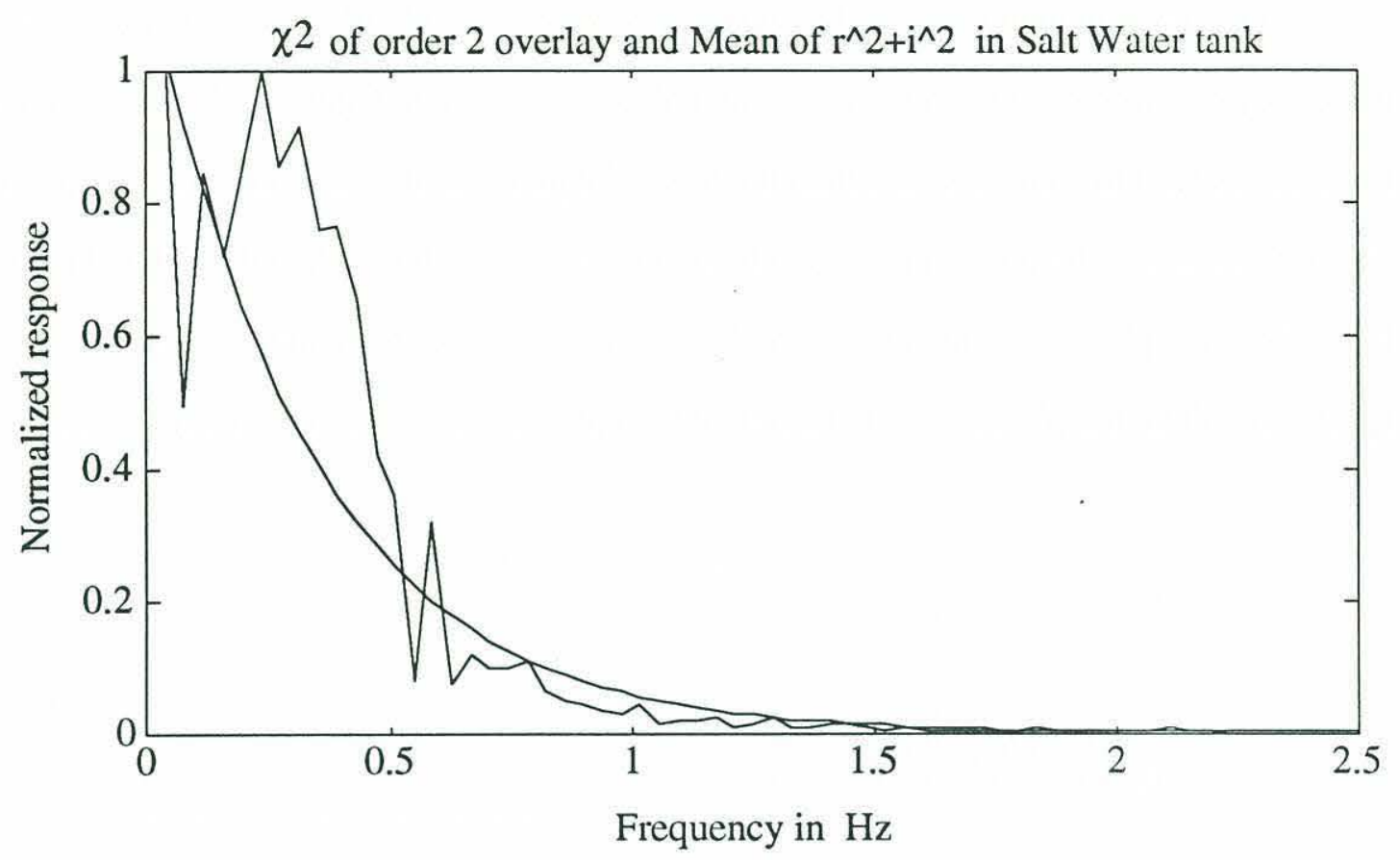

Figure 4.23

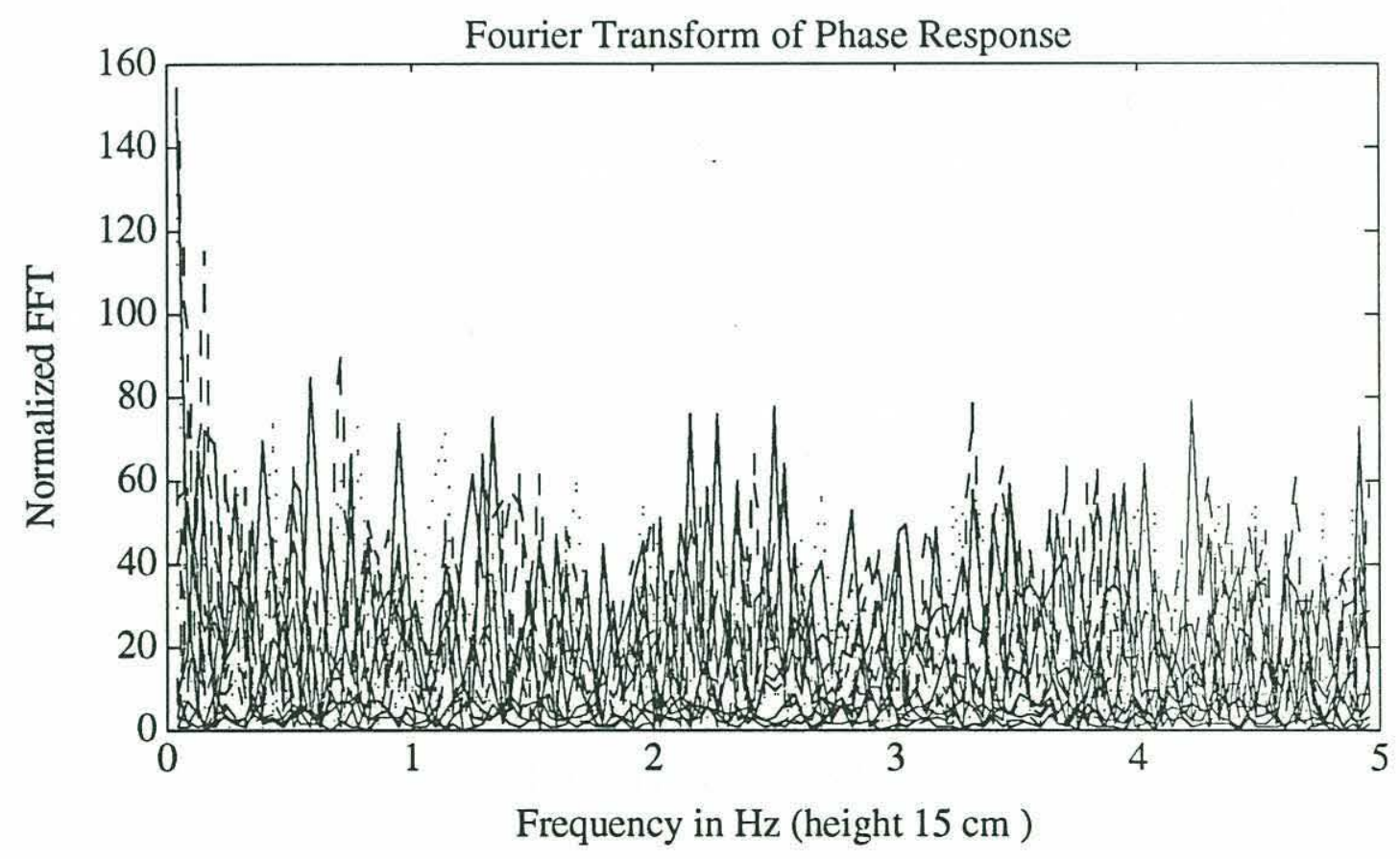

Figure 4.24 
The development of the turbulence can be seen fairly clearly in the overlaid plots of frequency distribution vs. power of the magnitude response in figure 4.25. The higher frequencies tend to form first and then the lower frequencies start to dominate the spectrum. This indicates that the plume appears to break up initially with smaller eddies breaking off first. Once the plume became more turbulent the larger eddies form and dominate the spectrum. When the plume is fully turbulent the spectrum looks to be closely Gaussian.

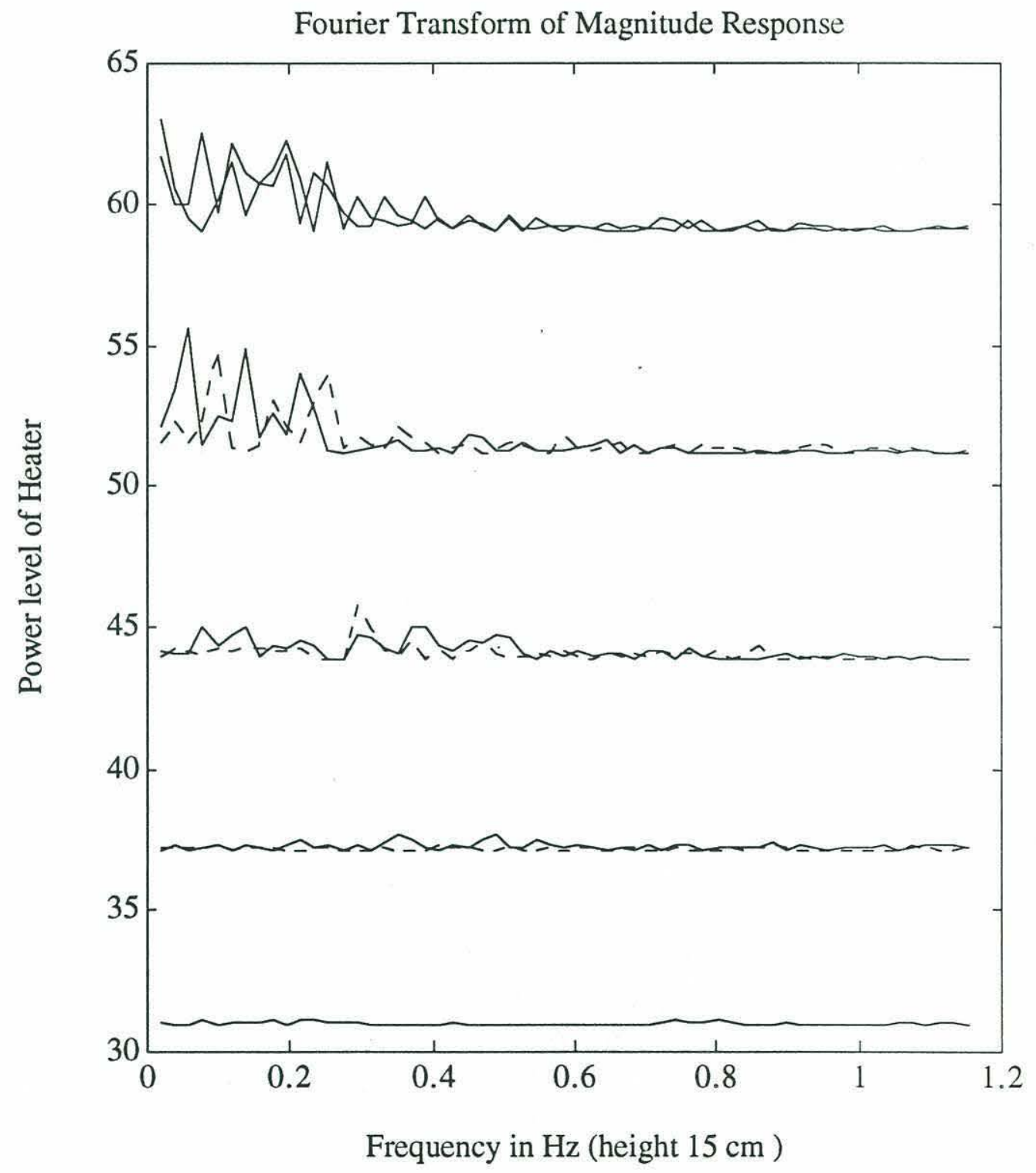

Figure 4.25 
The frequency spectrum of the modulated magnitude time series appears to be an excellent indication of the state of the plume. The development of the plume as power increases can also be seen from the spectrum. This method of analysis appears to be a fairly robust measure of the nature of the plume.

\subsubsection{Scintillation Index}

The scintillation index was also determined in the salt water tank for each height. The scintillation index for similar heights for the two tanks are overlaid on the plots below.

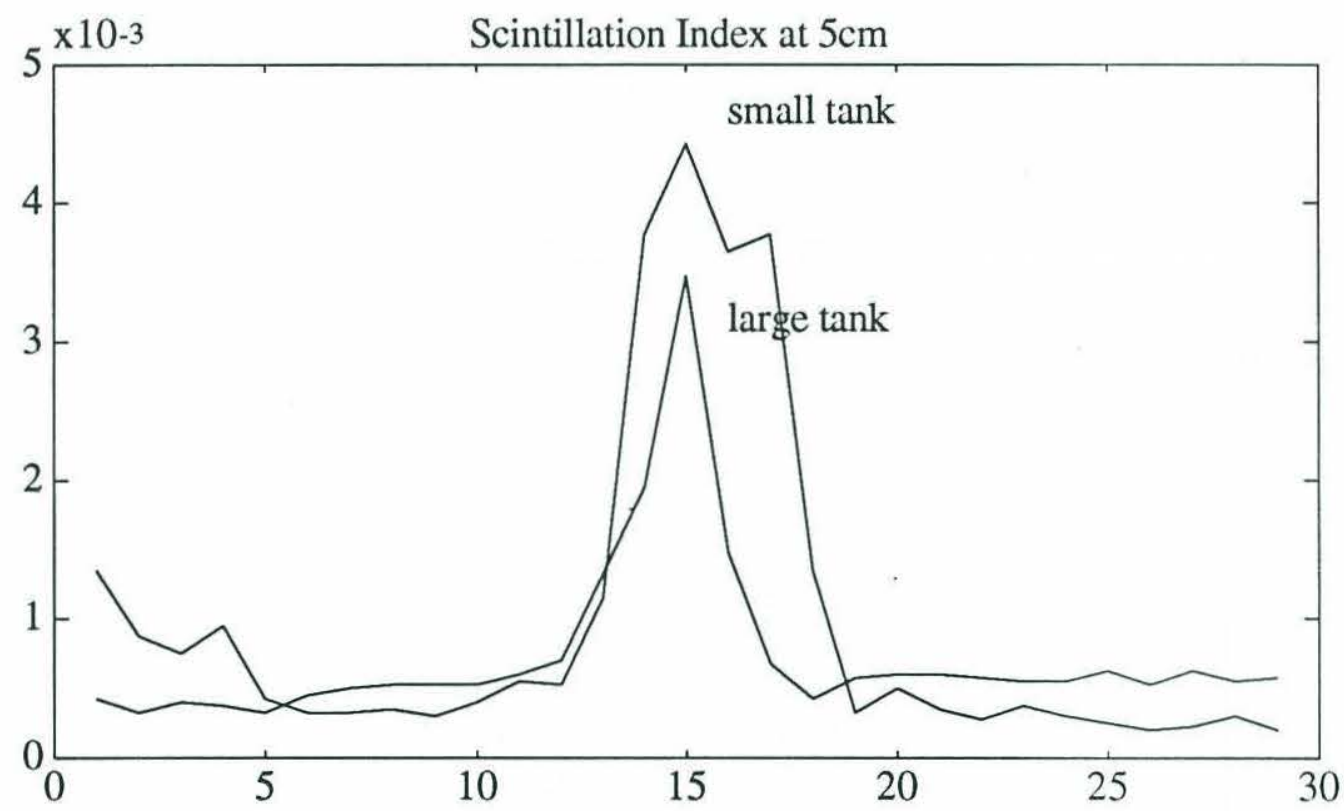

Run number (power $1=0$ watts, power $15=57$ watts, power $29=0$ watts)

Figure 4.26

The scintillation index for the small tank is generally larger as expected due to the increased amount of disturbances in the larger tank. However, the values are very close between the two tanks. Figure 4.26 shows the $5 \mathrm{~cm}$ scintillation response. 


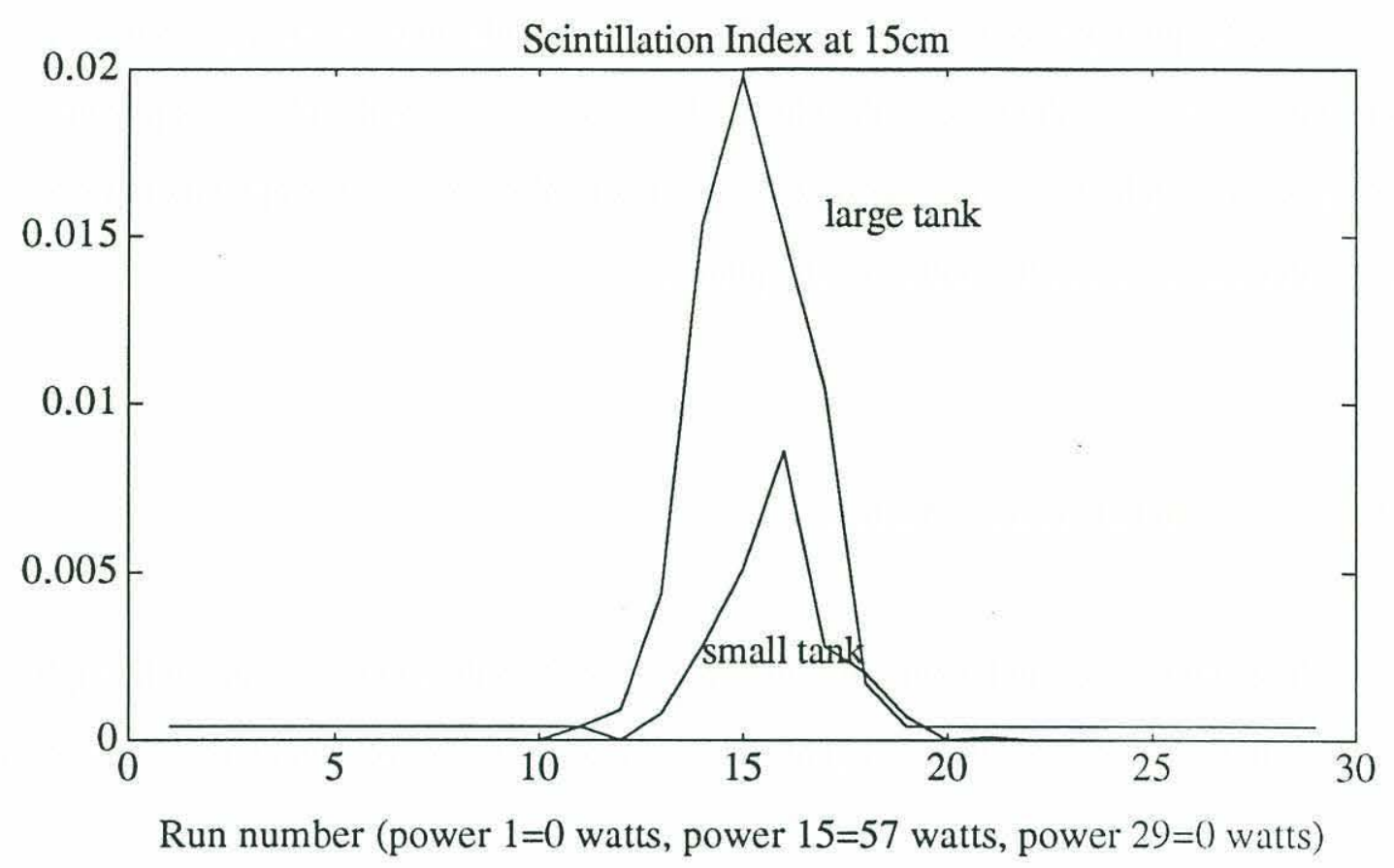

Figure 4.27

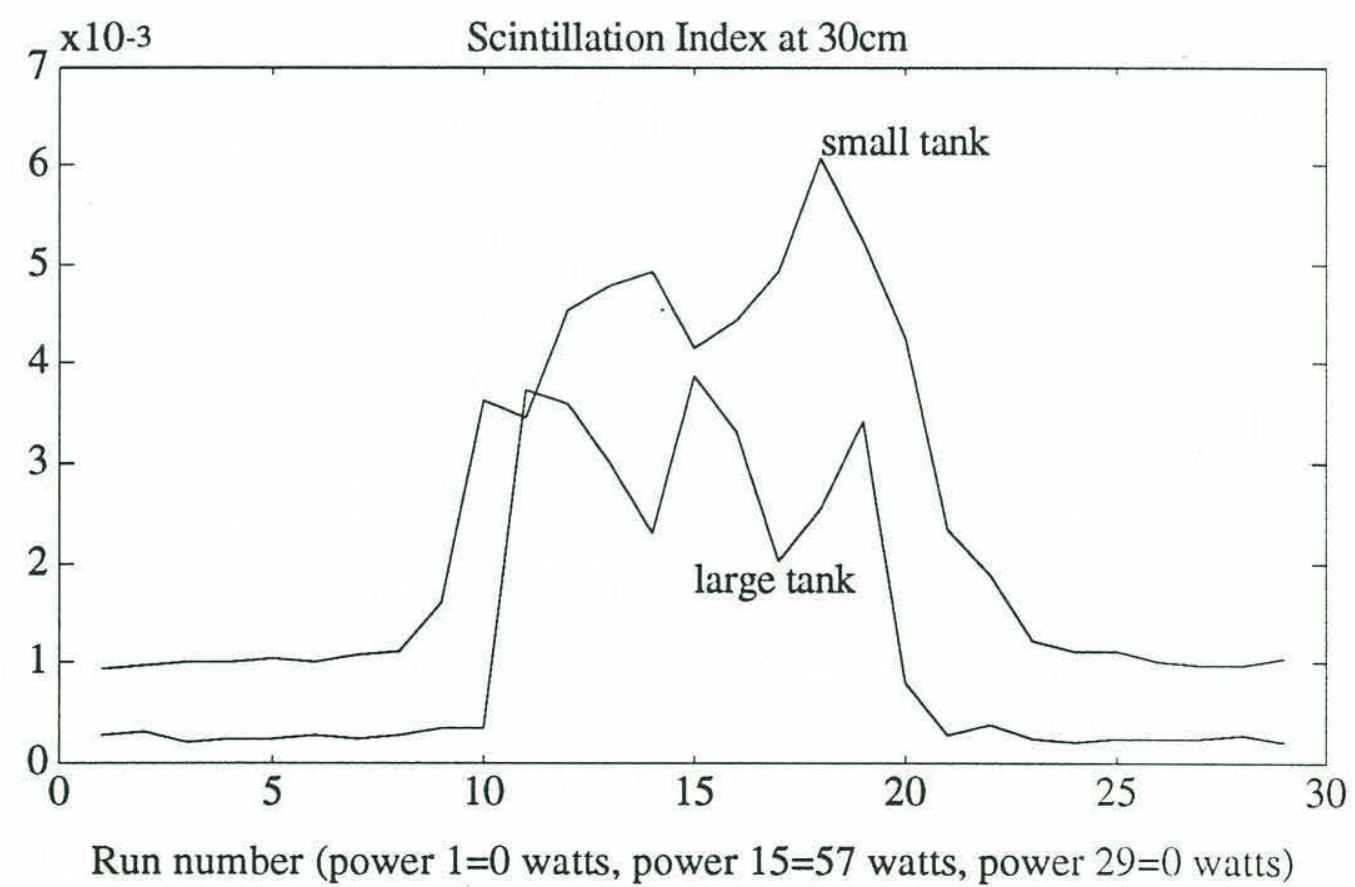

Figure 4.28 
Figure 4.27 confirms our earlier discussion of the $15 \mathrm{~cm}$ plume in the small tank. It appears that the plume in the small tank never reached a fully turbulent state. From the figure 4.27 the plume in the large tank may also not be fully turbulent.

The $30 \mathrm{~cm}$ plot in figure 4.28 illustrates the essentially saturated turbulence for the higher power levels. The plot also demonstrates the slightly reduced values for the larger tank.

Overall the scintillation index represents one of the most robust and easily achieved measurements of the plume structure discussed in this paper. Although the magnitude of the received signal can be reconstructed with careful analysis of the environmental data, it requires extensive manipulation. It also appears very difficult if not impossible without highly accurate measurements of the environment to recover the phase relationships. The frequency spectrum of the modulation appears to be a good indication of the nature of the plume, and is similar to the scintillation index in that it makes use of the variations in the time series of the magnitude.

\subsection{Scattering Function}

In order to use the information within this thesis for telemetry problems the scattering function of the plume needs to be determined. The scattering function is usually defined in terms of $s(\omega, r)$ and can be considered the average scatterer cross section ${ }^{41}$ for each pair of frequency and ranges. In this case only the frequency distribution was measured and as such the scattering function will be shown as $s(\omega)$. From Kennedy ${ }^{42}$ the $s(\omega)$ can also be seen as the frequency distribution of the correlation function, $R(t, \tau)$, of the received signal. This is sometimes termed the power spectral density $S(\omega)$. The mean correlation function of the signals from the fresh water tank at $30 \mathrm{~cm}$ are shown in figure 4.29. The power spectrum is also defined as the Fourier transform of the autocorrelation

\footnotetext{
${ }^{41}$ Kennedy, Robert S.,Fading Dispersive Communication Channels, (New York: Wiley-Interscience, 1969), pp. 14.

${ }^{42}$ Ibid, pp. 15.
} 

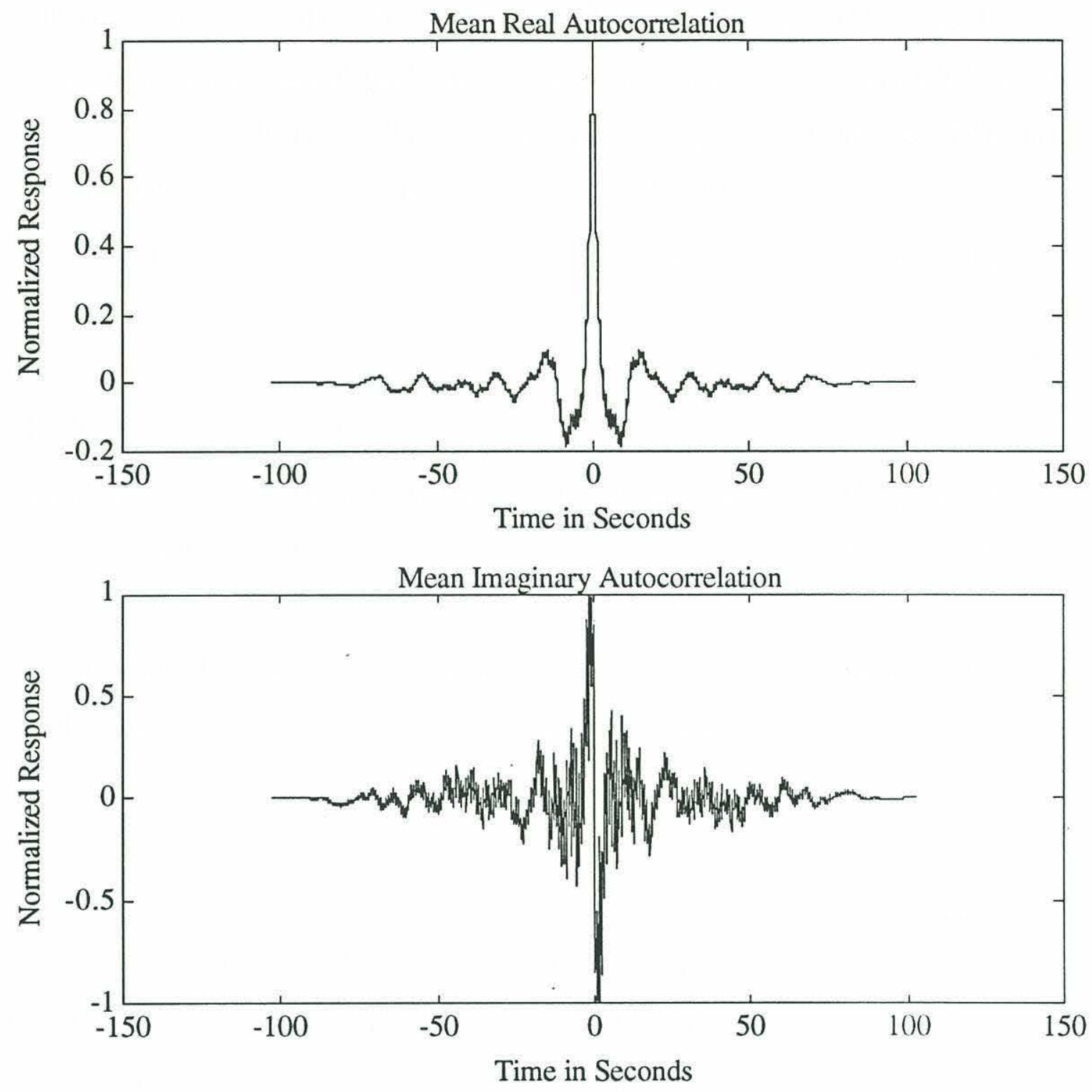

Figure 4.29

The estimated autocorrelation function shown in 4.29 produces the frequency response shown in figure 4.30 which may also be called the scattering function, $s(\omega)$. The Doppler spread, $B_{d}$, of the channel is defined in Proakis ${ }^{43}$ as the range of values over which the scattering function is non zero. For the demonstrated scattering function the Doppler

43John G. Proakis, Digital Communications (New York: McGraw-Hill, 1983), pp. 461 . 
spread is $0.2 \mathrm{~Hz}$. The coherence time from Proakis ${ }^{44},(\Delta t)_{c} \approx 1 / B_{d}$, is 5 seconds. This frequency spread is very small when compared to the usual telemetry transmission signals.

This response looks roughly like the frequency response of a damped oscillation. An over lay of this response is shown in figure 4.31. The scattering function could roughly be modeled with a damped oscillation response with a mean fiequency of 0.007 $\mathrm{Hz}$ and a $3 \mathrm{~dB}$ bandwidth of $0.04 \mathrm{~Hz}$. The transfer function for this model is:

$$
s(\omega)=\frac{1}{3.33-i 2.3 \omega+16.9 \omega^{2}}
$$

This appears to provide a useful model for analysis. The agreement is within the variance for most frequencies below $0.13 \mathrm{~Hz}$. As the frequency increases the noise of the system begins to dominate and the agreement becomes very poor. Figure 4.33 shows the exponential decay is present in higher frequencies for the model and the estimated scattering functions.

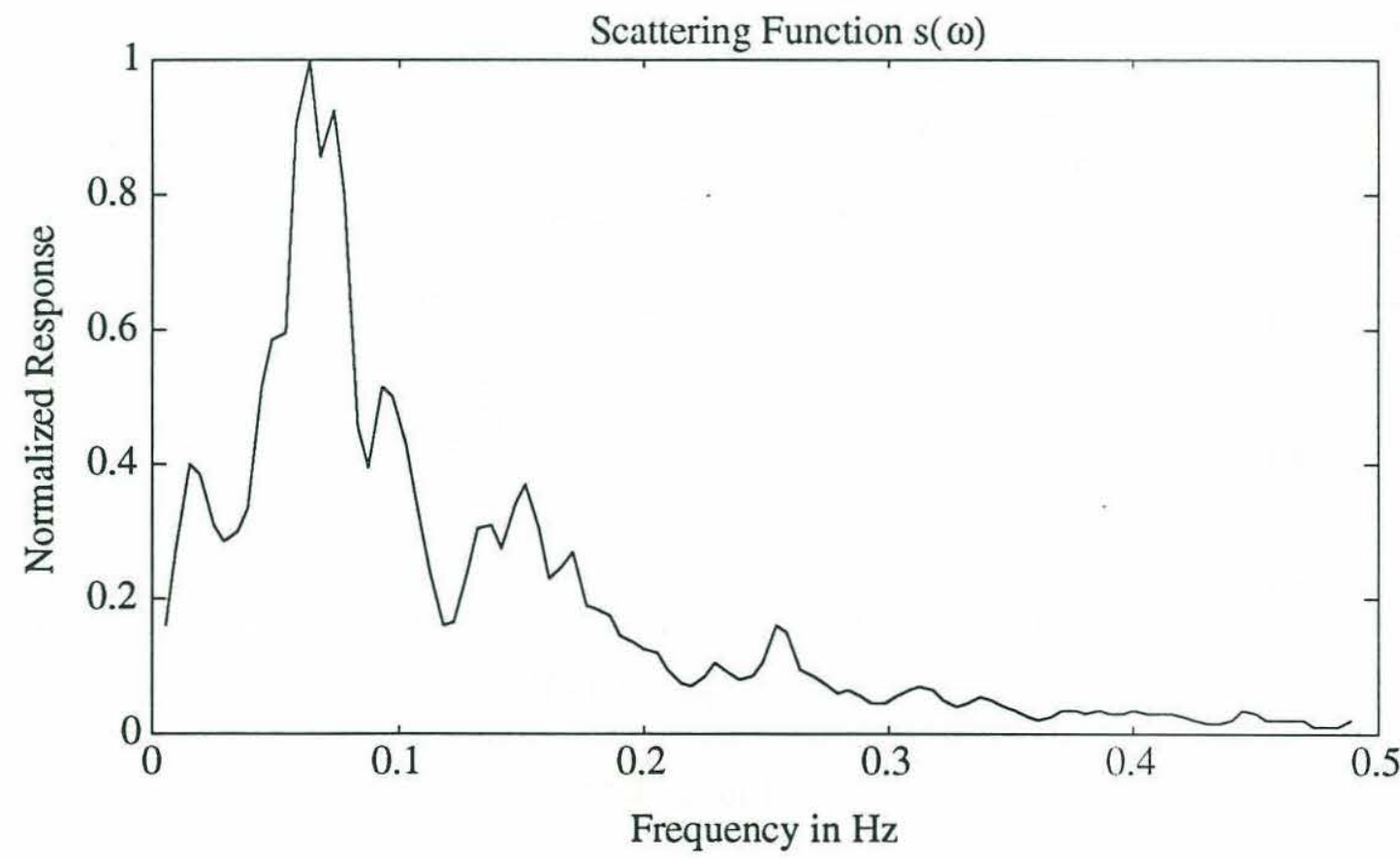

Figure 4.30

${ }^{44}$ Ibid. 


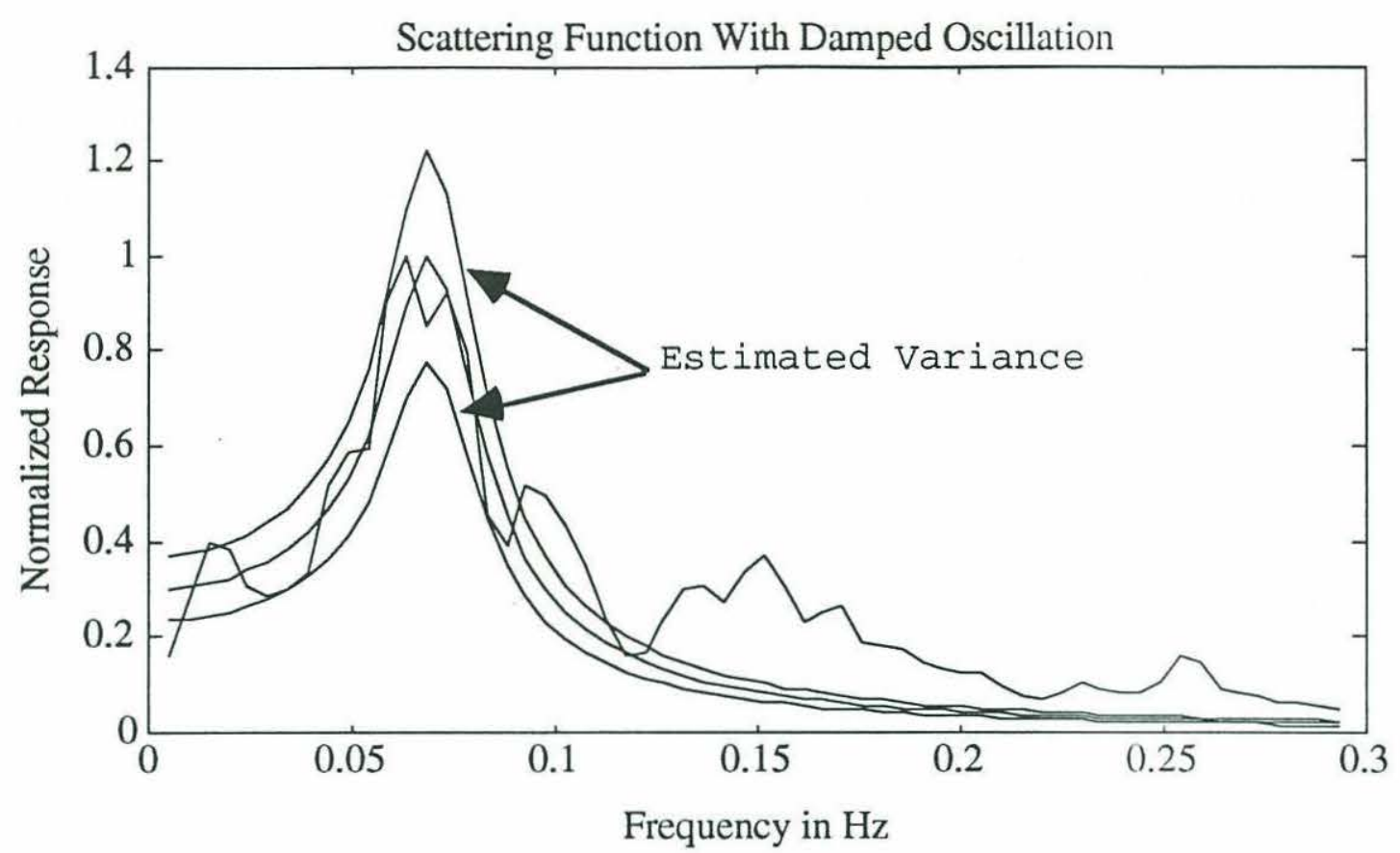

Figure 4.31

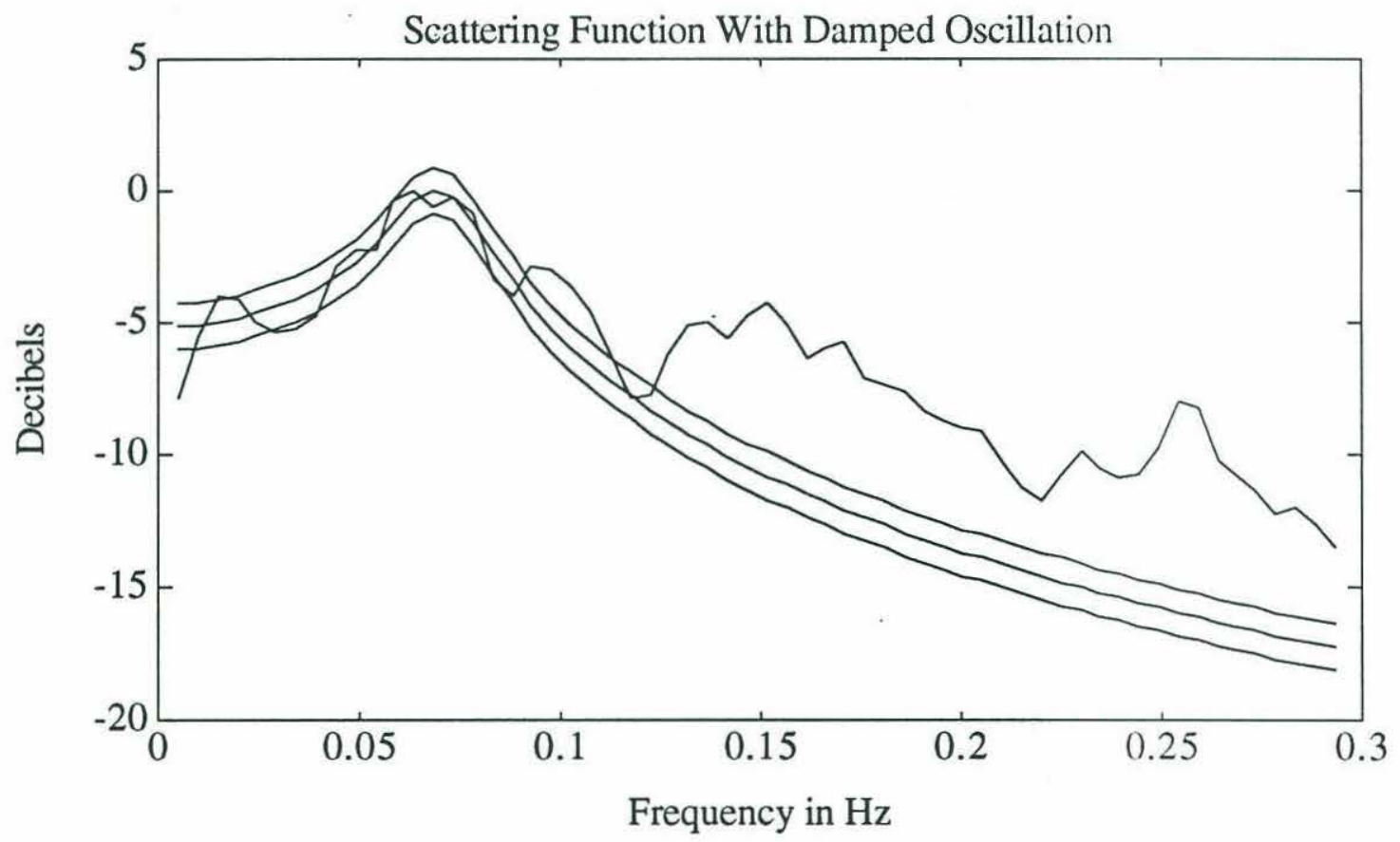

Figure 4.32 


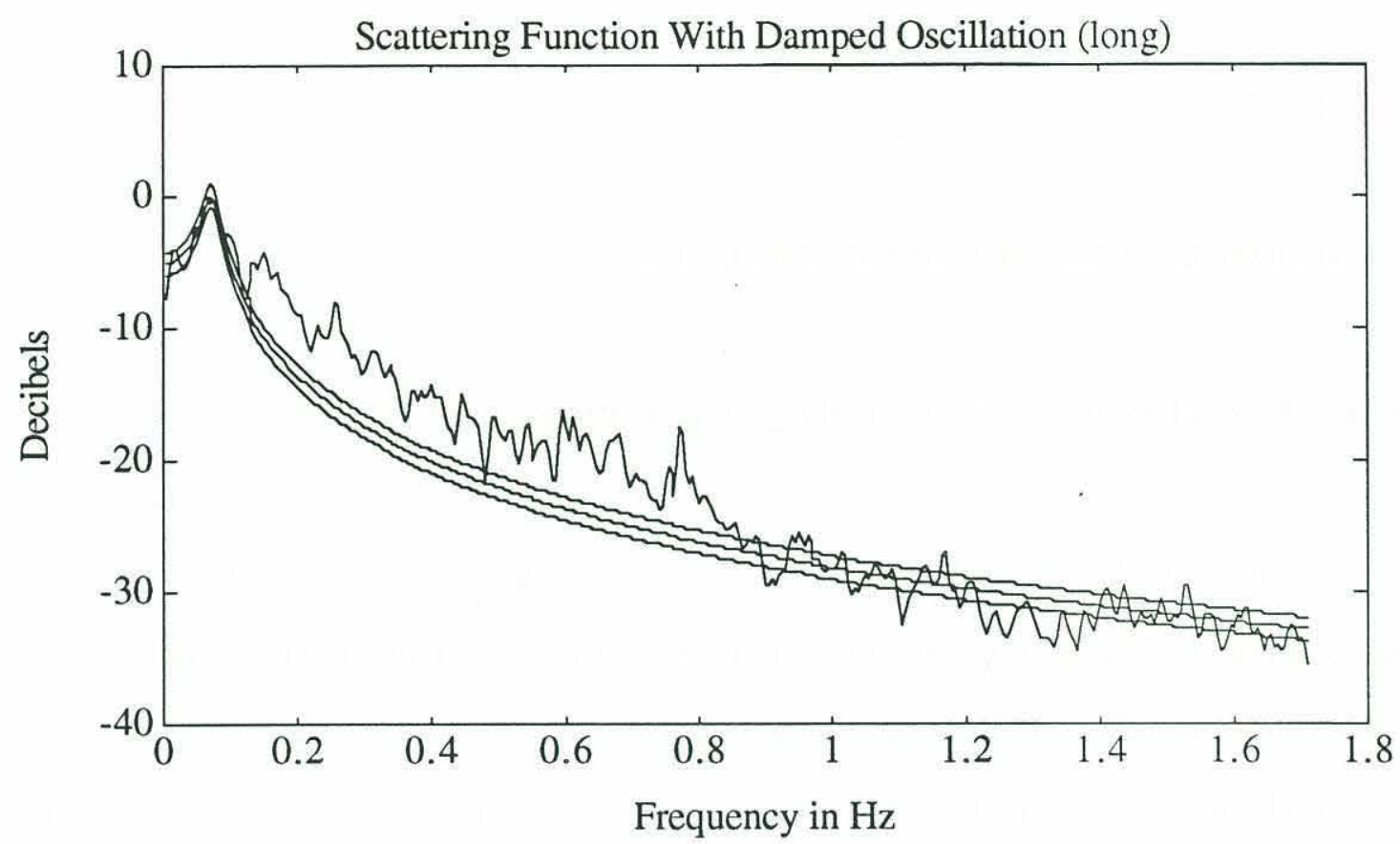

Figure 4.33 


\section{Chapter 5}

\section{Conclusions and Recommendations}

\subsection{Conclusions Concerning Application of Results}

The goal of this thesis has been to look at the effect of a buoyant plume on an acoustic signal. The plume was looked at in both its laminar and, at least partially, turbulent forms. Several tools were used to perform these observations. The use of the Born and Rytov approximations as models for the laminar plume was investigated. The scintillation index and the modulation frequency spectrum are investigated as robust measures of turbulence.

Specific results of this investigation included: the verification of linear spreading in a laminar plume; observation of a $\chi 2$ of order 2 distribution in the frequency domain of the magnitude square response; measurement of the Doppler spreading due to the plume of 0.2 $\mathrm{Hz}$; and observation of a damped oscillation frequency response model for the scattering function.

The Born approximation was determined to be a good measure of the magnitude of the received signal but did not follow the phase changes encountered. The Rytov approximation, at least to the first order, is not as good as the Born approximation in estimation of the magnitude response. However the Rytov approximation provides a good estimation of the phase response. Because of the phase response and the small absolute magnitude of the error in magnitude, the Rytov approximation is the preferred method for modeling this system.

The frequency distribution of the modulation of the received amplitudes indicate the nature of the plume. As the plume becomes more turbulent the spectrum more closely 
approaches a Gaussian distribution. As the plume develops in turbulence the spectrum initially rises at the higher frequencies and at the fully turbulent state the lower frequencies become dominant. This seems to indicate that the plume at first creates smaller eddies, and as the plume becomes more fully turbulent the larger eddies form and become prevalent.

From the experiments, the scintillation index proved to be the most robust estimate of the turbulence in the plume. It provided a measure of the transition to a turbulent plume in both the well controlled fresh water experiment and the large salt water tank experiment

in the presence of uncontrolled factors. The plume should be interrogated by a sufficiently strong signal and the pulse train should be of sufficient length to ensure the ability to discern the signal from the particulatly scattered signal. In addition the pulse train need only be of a length to allow for the necessary number of cycles for stable statistical properties. Hopefully, the length of the pulse train will be significantly less than the period of the internal waves in the media through which the sampling occurs.

The tools used in this thesis provide a range of useful methods. Using the Rytov approximation and the magnitude and phase responses of the signal in a controlled experiment can provide great insight into the plume parameters. The frequency response and the scintillation index provide insight into the controlled experiment as well, but really offer the most robust tools for observing a plume under adverse conditions.

\subsection{Recommendations for Further Research}

The overall conclusion of the research conducted is that there needs to be more research. The experiments discussed above touched on many aspects of the thermal plume without fully delving into any single area. Several experiments immediately suggest themselves. 
A detailed study of the plume at various heights using multiple sources and receivers simultaneously on the same plume. This should be followed by a detailed temperature probe investigation of the plume at different heights. This would provide specific constants, similar to those available in the atmospheric modeling (eqns. 8 and 9), of buoyant plumes. This would allow specific modeling of the plume based on the acoustic interrogation. With limited data under controlled conditions the linear spreading of the laminar plume was verified. More turbulent data under a controlled experiment should verify the $-5 / 3$ spreading of the turbulent plume.

An experiment placing several sources and receivers around a buoyant plume at sea with a set geometric orientation to provide remote sampling of the plume would use the observed results of this experiment. By using a pulsed signal, such as used in this experiment, discrete samples of the plume can be taken and the robustness of the scintillation index as a measure of turbulence of a plume in a natural environment can be assessed.

The submarine spring plume is a common buoyant plume available for monitoring. Colbourne and Hay ${ }^{45}$ conducted a study of one such plume using a submersible and acoustic backscattering. The source was roughly 5 meters in diameter with vertical velocities near $30 \mathrm{~cm} / \mathrm{s}$. Salinity measured at one meter varied up to $5 \mathrm{ppt}$ while temperature varied only about $0.2^{\circ} \mathrm{C}$. This leads to a peak velocity differential in the plume of $5.2 \mathrm{~m} / \mathrm{s}$ due to the dominant effect of the change in salinity for these values. A change of $5.2 \mathrm{~m} / \mathrm{s}$ over 5 meters produces a very significant effect on the propagated signal and should be easily measurable (the experiment in this paper produced roughly $15 \mathrm{~m} / \mathrm{s}$ change over 2 $\mathrm{cm})$.

\footnotetext{
${ }^{45}$ Eugene B. Colbourne and Alex E. Hay, "An Acoustic Remote Sensing and Submersible study of an Arctic Submarine Spring Plume, "Journal of Geophysical Research, 95, No. C8 (15 August 1990), 13219-13234.
} 
In recent years another source, the hydrothermal vent fields, has been subject to considerable research. Little, Stolzenbach, and Von Herzen ${ }^{46}$ used DSR/V Alvin to obtain measurements in a vent field and obtained vertical velocities near $5-10 \mathrm{~cm} / \mathrm{s}$ and temperature peaks slightly greater than $2^{\circ} \mathrm{C}$ at 24 meters above the field. Similarly, Speer and Rona ${ }^{47}$ arrived at similar values in their models of hydrothermal plumes. These fields are highly turbulent and extend over 100x100 meter areas. On a smaller scale, single hydrothermal openings are on the order of $2-8 \mathrm{~cm}$. Temperature variations closer to the vents are from $30-420^{\circ} \mathrm{C}$ for hot smokers and $5-30^{\circ} \mathrm{C}$ for diffuse flow from cracks. ${ }^{48}$ There currently exists no remote sensing systems monitoring any of these fields on a continuous basis.

The possibility of using forward scattering across one of these vent fields as an economical means of monitoring the activity of the field offers an obvious application of this research. A small network of several transducers and hydrophones would offer different paths for monitoring a large area.

Finally, the nature of buoyant plumes due to variation in the layered media by a horizontal body motion can be investigated using this line of sight method. The time series plots of signal response obtained from an experiment involving the insonification of the wake left by $22.9 \mathrm{~cm}$ diameter by $122 \mathrm{~cm}$ slender body 49 appears to be similar to the type of responses obtained in this experiment. Analysis by both the scintillation index and the frequency transformation of the time series discussed above may reveal significant information. This type of frequency analysis has not been done in the available literature.

\footnotetext{
${ }^{46}$ Sarah Little, Keith Stolzenbach, and Richard P. Von Herzen, "Measurements of Plume Flow From a Hydrothermal Vent Field," Journal of Geophysical Research,92, No.B3 (10 March, 1987), 2587-2596. 47 Kevin G. Speer and Peter A. Rona, "A Model of an Atlantic and Pacific Hydrothermal Plume," Journal of Geophysical Research, 94, No.c5(15 May $1989), 6215-6219$. 


\section{Bibliography}

[1] Artel'nyi, V. V., and M. A. Raevskii. "Multiple Scattering of Low-frequency Sound Waves by Ocean Turbulence." Soviet Phys. Acoust., 33, No. 1 (JanuaryFebruary 1987), 4-7.

[2] Baerg, William, and W. H. Schwarz. "Measurements of the Scattering of Sound from Turbulence." The Journal of The Acoustical Society of America, 39, No. 6 (1966), 1125-1132.

[3] Batchelor, G. K. " Heat Convection and Buoyancy Effects in Fluids." Quarterly Journal of The Royal Meteorological Society, 80 (1954), 339-358.

[4] Chernov, Lev. Wave Propagation in A Random Medium, Trans. R. A. Silverman. New York: McGraw-Hill, 1960.

[5] Clay, Clarence S., and Herman Medwin. Acoustical Oceanography: Principles and Applications. New York: John Wiley \& Sons, 1977.

[6] Colbourne, Eugene B., and Alex E. Hay. "An Acoustic Remote Sensing and Submersible Study of an Arctic Submarine Spring Plume." Journal of Geophysical Research, 95, No. C8 (August 15, 1990), 13219-13234.

[7] Duda, Timothy P. "Modeling Weak Fluctuations of Undersea Telemetry Signals." IEEE Journal of Oceanic Engineering, 16, No. 1 (January 1990), 3-11.

[8] Flatté, Stanley M. "Wave Propagation Through Random Media: Contributions From Ocean Acoustics." Proceedings of The IEEE, 71, No. 11 (November 1983), 1267-1294.

[9] Gavrilenko, V. G., M. S. Kovner, and A. I. Mart'yanov. "Wave Scattering by a Turbulent Jet." Soviet Phys. Acoust., 23, No. 5, (September-October 1977), 404406.

[10] Goodman, L., J. Oeschger, and D. Szargowicz. "Ocean Turbulence Study: Acoustic Scattering From a Buoyant Axisymmetric Plume." Journal of The Acoustical Society of America, 91, No. 6 (June 1992), 3212-3227.

[11] Harris, Fredrick J. "On The Use of Windows for Harmonic Analysis with the Discrete Fourier Transform." Proceedings of the IEEE, 66, No. 1 (January 1978), 51-83.

[12] Hay, Alex E. "Sound Scattering From a Particle-laden, Turbulent Jet." Journal of The Acoustical Society of America, 90, No. 4 (October 1991), 2055-2073.

[13] Ishimaru, Akira. Wave Propagation and Scattering in Random Media. 2 vols. New York: Academic Press, 1978.

[14] Kinsler, Lawrence E., et al. Fundamentals of Acoustics, 3rd ed. New York: John Wiley \& Sons, 1982. 
[15] Kraichnan, Robert H. "The Scattering of Sound in a Turbulent Medium." The Journal of The Acoustical Society of America, 25, No.6 (November 1953), 10961104.

[16] Korman, M. S., and R. T. Beyer. "The Scattering of Sound by Turbulence in Water." The Journal of The Acoustical Society of America, 67, No. 6 (June 1980), 1980-1987.

[17] Little, Sarah A., Keith D. Stolzenbach, and Richard P. Von Herzen.

"Measurements of Plume Flow From a Hydrothermal Vent Field." Journal of Geophysical Research, 92, No. B3 (March 10, 1987), 2587-2596.

[18] Mintzer, David. "Wave Propagation in a Randomly Inhomogeneous Medium. I." The Journal of The Acoustical Society of America, 25, No.5 (September 1953), 922-927.

[19] Morse, Philip M. and K. Uno Ingard. Theoretical Acoustics. Princeton: Princeton University Press, 1968.

[20] Oeschger, John Willet. Acoustic Scattering From a Laminar Plume. Dissertation: University of Rhode Island, 1993.

[21] Oeschger, John Willet. Telephone conversation, 24 June 1993.

[22] Papoulis, Athanasios. Probability, Random Variables, and Stochastic Processes. New York: McGraw-Hill, 1991.

[23] Pelech, I., G. G. Zipfel, and R. L. Holford. "A Wake-scattering Experiment in Thermally Stratified Water." The Journal of The Acoustical Society of America, 73, No. 2 (February 1983), 528-538.

[24] Proakis, John G. Digital Communications. New York: McGraw-Hill, 1983.

[25] Rasmussen, Robert A. "Remote Detection of Turbulence From Observations of Reverberation Spectra." The Journal of The Acoustical Society of America, 63, No. 1 (January 1978), 101-110.

[26] Rona, P.A. and D. R. Palmer. "Acoustic Imaging of Hydrothermal Plumes, East Pacific Rise, $21^{\circ}$ N. $109^{\circ}$ W." Geophysical Research Letters, 18 , No. 12 (December 1991), 2233-2236.

[27] Rouse, H., C. Yih, and H. W. Humphreys. "Gravitational Convections from a Boundary Source." Tellus, 4 (1952), 201-210.

[28] Speer, Kevin G. and Peter A. Rona. "A Model of an Atlantic and Pacific Hydrothermal Plume." Journal of Geophysical Research, 94, No. C5 (May 15, 1989), 6213-6220.

[29] Stanton, T. K. "Sound Scattering by Cylinders of Finite Length. I. Fluid Cylinders." The Journal of The Acoustical Society of America, 83, No.1 (January 1988), 55-63.

[30] Tatarski, V. I. Wave Propagation in a Turbulent Medium. New York: Dover Publications, 1961. 
[31] Tarng, J. H. and C. C. Yang. "Acoustic Beam Propagation in a Turbulent Ocean." The Journal of The Acoustical Society of America, 84, No. 5 (November 1988), 1808-1812.

[32] Tennekes, H. and J. L. Lumley. A First Course in Turbulence. Cambridge, Massachusetts: The MIT Press, 1987.

[33] Townsend, A. A. "Entrainment and the Structure of Turbulent Flow." Journal of Fluid Mechanics, 41, No. 1 (1970), 13-46.

[34] Turner, J. S. Buoyancy Effects in Fluids. Cambridge: Cambridge University Press, 1973.

[35] Wenzel, Alan R. and Joseph B. Keller. "Propagation of Acoustic Waves in a Turbulent Medium." The Journal of The Acoustical Society of America, 50, No.3, Part 2 (1971), 911-920.

[36] Wittek, G. "Amplitudenmodulation von Ultraschallwellen durch Turbulente Wasserströmungen." Acustica, 27 (1972), 7-14. 